ON ENDOGENOUS COMPETITIVE

\title{
BUSINESS CYCLES
}

\section{Jean-Michel GRANDMONT}

$N^{\circ} 8316$

June 1983, Revised September 1983 
This paper develops an example in which persistent deterministic business cycles appear in a purely endogenous fashion under laisser faire. These cycles are not attributable to exogenous "shocks" nor to any variation of policy since there are none in the model. Markets clear in the Walrasian sense at every date, and traders have perfect foresight along the cycles.

The origin of these cycles is the potential conflict between the wealth effect and the intertemporal substitution effect that are associated to real interest rate movements. Business cycles appear in particular when the degree of concavity of a trader's utility function is sufficiently higher for old agents than for younger ones. The techniques employed to study the occurrence and the stability of such business cycles are borrowed partly from recent mathematical theories that have been constructed by using the notion of the "Hopf's bifurcation" of a dynamical system in order to explain the emergence of cycles and the transition to turbulent ("chaotic") behaviour in physical, biological or ecological systems.

The equilibrium level of output is shown to be negatively related to the equilibrium level of the real interest rate. A similar relation exists (but in the opposite direction) between equilibrium real money balances and real interest rates. These relations hold both in the long run, i.e. along business cycles, and in the short run, i.e. on the transition path, and whether movements of the real interest rate are anticipated or not. The basic ingredient there is the condition that older agents have a higher marginal propensity to consume leisure. 
Monetary policy by means of nominal interest payments is shown to be extremely effective. A permanent change of the rate of growth of the money supply by these means will be superneutral, Yet, there exists a very simple deterministic countercyclical policy that enables monetary authorities to stabilize completely business cycles and to force the economy back to the unique (Golden rule) stationary state. Due to the nonlinearity of the model such a policy affects not only the variances of real equilibrium magnitudes but also their means. 
Cet article développe un exemple dans lequel des cycles économiques déterministes apparaissent de manière purement endogène. Ces cycles ne sont dus ni à des "chocs" exogènes ni à des changements de politique économique puisqu'il n'y en a aucun dans le modèle. Les marchés s'équilibrent au sens Walrasien à chaque date et les agents prévoient correctement l'avenir le long des cycles.

L'origine de ces cycles est le conflit potentiel entre l'effet de richesse et l'effet de substitution intertemporel associés à une variation du taux d'intérêt réel. Des cycles apparaissent en particulier lorsque la concavité des fonctions d'utilité des agents est plus prononcée lorsqu'ils sont jeunes que lorsqu'ils sont plus agés. Les techniques utilisées pour étudier l'existence et la stabilité de ces cycles sont empruntées à des théories mathématiques récentes qui ont été développées en employant la notion de "bifurcation de Hopf" afin d'expliquer l'apparition de cycles et la transition vers la turbulence (1e "chaos") dans les systèmes physiques, biologiques ou écologiques.

Le niveau de production d'équilibre est dans ce modèle relié négativement au niveau d'équilibre du taux d'intérêt réel. Une relation analogue existe (mais dans la direction opposée) entre niveaux d'équilibre des encaisses monétaires réelles et du taux d'intérêt réel. Ces relations sont valables non seulement dans le long terme, c'est-à-dire le long des cycles, mais aussi dans le court terme, c'est-à-dire le long du sentier de transition, que les mouvements du taux d'intérêt réel soient anticipés ou non. L'hypothèse principale ici est que les agents plus agés aient une propension marginale à consommer du loisir plus importante. 
Il est montré que la politique monétaire au moyen de paiements de taux d'intérêts nominaux est très efficace. Un changement permanent du taux d'intérêt nominal est bien sûr superneutre. Cependant, il existe une politique contracyclique très simple qui permet aux autorités monétaires de stabiliser complètement cette économie et de la ramener à l'unique état stationnaire correspondant à la Règle d'Or. Etant donné le caractère nonlinéaire du modèle, cette politique altère non seulement la variance des quantités réelles d'équilibre mais aussi leurs moyennes. 


\section{ON ENDOGENOUS COMPETITIVE}

\section{BUSINESS CYCLES}

Jean-Michel GRANDMONT *

The belief that the long run equilibrium of a competitive monetary economy that does not experience any exogenous shocks - whether originating from the external environment or from policy - should be modelled as a state that is stationary or perhaps growing at a constant rate, seems to be deeply rooted in the mind of economists.

The most outspoken believers in the market's invisible hand go indeed as far as claiming that any departure from a long run Walrasian equilibrium should be regarded as purely transitory and that accordingly the basic tendencies of a competitive economy may be represented adequately by such a "Classical" stationary equilibrium. The most recent reformulation of the Classical approach has been to model economic fluctuations by adding random shocks to the deterministic stationary state and to

* CEPREMAP, Paris. This paper has been prepared for the IMSSS Summer Workshop at Stanford University. The financial support of the National Science Foundation , of the French Commissariat Général du Plan and the University of Lausanne is gratefully acknowledged.

I wish to thank very much Rose Anne Dana and Pierre Malgrange who introduced me to the mathematics of the subject : without them, this research could not have been completed. Christian Gouriéroux deserves also a special mention for his decisive advice in connection to the stability issue of Section 3, Rose Anne Dana and Dominique Lévy ran the computer experiment reported in Section 4. Their help is gratefully acknowledged, Earlier simulations by Alain Morineau were also very valuable.

I had stimulating conversations with and received useful comments from many colleagues at various stages of this work. I wish to tharik them a 11, and especially Philippe Aghion, Albert Ando, Jean-Pascal Benassy, Robert Boyer, David Cass, Jean-Pierre Danthine, Frank Hahn, Guy Laroque and Yves Younès. 
underscore the role of incomplete (and asymmetric) information in the influence of economic policy on real equilibrium variables. The outcome of this reformulation is a model that preserves very cleverly stationarity while incorporating in the analysis something that looks like business cycles (Barro (1981), Kydland and Prescott (1982), Lucas (1972, 1975, 1977, 1980, 1981), Sargent and Wallace (1975)) ${ }^{1}$. An important implication of many, but not a11, of these models is that the systematic (deterministic) component of economic policy can have no real effect whenever it is anticipated by the private sector.

The arguments put forward by the opposing (Keynesian) school appear often, by contrast, almost exclusively defensive. Proponents of this school seem to accept in effect the theoretical validity of the claim according to which the long run equilibrium positions of a competitive economy may be described by (deterministic or stochastic) stationary states. They tend to question primarily the practical relevance, for the description of short run and medium run phenomena, of the mere notion of a long run stationary equilibrium and of its underlying assumptions. The list is long : prices cannot move fast enough to clear markets, anticipations adjust only slowly, New Classical macroeconomic models rely upon extreme1y specific assumptions concerning the distribution of information, the Classical stationary state may be unstable or convergence to it may be so slow that it becomes practically irrelevant in calendar time, and so on ${ }^{2}$.

The purpose of this work is to demonstrate that, by contrast to currently accepted views, a competitive monetary economy of which the environment is stationary may undergo persistent and large deterministic

I See also Grandmont and Hildenbrand (1974).

2

For an excellent account of the long feud between Keynesian and (01d, Neo - or New) Classical economists, see e.g. Tobin (1980). 
fluctuations under laisser faire. That these cyclical fluctuations may display moreover the sort of correlations that recent Classical macroeconomic models have seeked to incorporate, without having to make the ad hoc assumption that cycles are due to exogenous shocks. And finally, that the Government, in the face of such autonomous deterministic fluctuations, has indeed in principle the power to stabilize the economy by implementing simple deterministic - and publicly known - countercyclical policies.

Although one of the goals of the present work is to develop concepts and methods that can be applied, it is hoped, to a larger class of situations, the analysis will proceed by studying a particular example, i.e. an overlapping generations model very much alike the model developed by R.J. Lucas in his seminal paper (1972), with the noticeable difference that we shall assume that the economy is not subjected to any shock of any sort. Business deterministic cycles will be shown to appear in a purely endogenous fashion under laisser faire. Markets will be assumed to clear in the Walrasian sense at every date, and traders will have perfect foresight along the cycles.

The origin of these endogenous deterministic cycles will be seen to be the potential conflict between the wealth effect and the intertemporal substitution effect that are associated to real interest rate movements. Business cycles will emerge in particular when the degree of concavity of a trader's utility function - which we shall measure, although there is no uncertainty in the model, by the the so-called Arrow-Pratt "relative degree of risk aversion" is sufficiently higher for old agents than for younger 
ones. An important outcome of the analysis will be that cycles of different periods will typically coexist - in some cases, there may be a countable number of these. The techniques employed to study the occurrence and the stability of such business cycles will be borrowed partly from recent mathematical theories that have been constructed by using the notion of the "Hopf's bifurcation" of a dynamical system in order to explain the emergence of cycles and the transition to turbulent ("chaotic") behaviour in physical, biological or ecological systems ${ }^{2}$.

The equilibrium level of output will be shown to be negatively related to the equilibrium level of the real interest rate. A similar relation exists (but in the opposite direction) between equilibrium real

The idea that endogenous deterministic cycles may emerge in an overlapping generations mode 7 is already present in the literature. For instance David Gale (1973) presents a numerical example of a cycle of period 2, while David Cass (1980) discusses graphically the possibility of their occurrence. Moreover, the deterministic business cycles that are the subject of this paper may be assimilated to what has been called recently "sunspots" equilibria. The analysis of sunspot equilibria has been started by Karl Shell (1977) and later developed by C. Azariadis (1981) and D. Cass and K. Shell (1981, 1983). Independentiy of the present work, a forthcoming paper by $C$. Azariadis and R. Guesnerie (1983) that I have not yet seen, establishes that there are sunspots equilibria if and only if deterministic cycles exist.

Finally, a recent paper by $P$. Diamond and D. Fudenberg (1983) provides an example of an endogenous rational expectations business cycle in a search equilibrium model, in an otherwise stationary environment.

2

One important mathematical reference in this field is collet and Eckmann (1980). For a stimulating review of various applications of the theory, see May. (1976). Part of this theory has been already applied in economics or game theory, in particular by Benhabib and Day (1981, 1982), Dana and Malgrange (1981), Day (1982, 1983), Jensen and Urban (1982), Rand (1978). The results of this theory that seemed (to me) relevant and useable by economic theorists are reviewed in Grandmont (1983b). 
money balances and real interest rates. These relations hold both in the long run, i.e. along business cycles, and in the short run, i.e. on the transition path, and whether movements of the real interest rate are anticipated or not. The basic ingredient there will be the condition that older agents have a higher marginal propensity to consume leisure.

Finally, monetary policy by means of nominal interest payments will be shown to be extremely effective. A permanent change of the rate of growth of the money supply by these means will be seen to be superneutral. Yet, it will be shown that there exists a very simple deterministic countercyclical policy that enables monetary authorities to stabilize completely business cycles and to force the economy back to the unique (Golden rule) stationary state. Due to the nonlinearity of the model, such a policy affects not only the variances of real equilibrium magnitudes but also their means. The central point here is that there are typically many long run periodic equilibria that coexist under laisser faire, and that policies may be designed which force the economy to settle at only one of these - here the stationary state ${ }^{1}$.

\section{The paper is organized as follows. We specify in Section 1}

the structure of the model and study there the traders' microeconomic behaviour. The dynamic system describing the evolution over time of the economy as well as long run periodic equilibria are defined in Section 2. The issue of the stability of these periodic equilibria is partly analysed in Section 3. The existence, the multiplicity, and

The idea that there may be a large number of "rational expectations" or perfect foresight equilibria in a monetary economy and that accordingly, one possible role of policy is to select one of these, is also already present in the literature. In particular, this fact has been well known by theorists who worked with the overlapping generations model. The point has been most forcedfully reiterated recently by F. Hahn (1982). 
the bifurcations of periodic competitive equilibria are investigated systematically in Section 4 . The long run and short run relationships between equilibrium output or real balances, and anticipated or unanticipated real interest rates are established in Section 5. Finally, the impact of monetary policy through deterministic money transfers is dealt with in Section 6. A few concluding remarks are given in Section 7, while some proofs are gathered in a separate Appendix.

Section 4 is the most technical, and although it is in some respects the most interesting one, the nonmathematically oriented reader may skip it on a first reading. Section 5, and to a large extent, Section 6 , can be read right after the first two sections of the paper. 
1. BEHAVIORAL ASSUMPTIONS.

We shall use the simple structure of an overlapping generation model, with a constant pupulation and without bequests, in which agents live two periods only. For simplicity we shall assume that all agents are identical, or equivalently that there is a single member in each generation. There will be accordingly two agents in every period, one "young" and one "old". The model involves one perishable consumption good, which is produced from the labour that is supplied by consumers. There is no production lag, and producing one unit of output requires one unit of labour. Young consumers have the opportunity to save part of their income in each period by holding a nonnegative money balance. For the most part of the paper, the money stock will be assumed to be constant over time. It will be denoted $M>0$.

At each date $t$, there are competitive spot markets for the consumption good, for labour and for money. The money price of the good and the money wage rate will be denoted $p_{t}$ and $w_{t}$, respectively. However, in equilibrium, a positive amount of the good is consumed and therefore produced (think of the old agent who has a positive money balance to spend on the good market at every date). Profit maximization in the production sector implies then the equality of the equilibrium real wage and the equilibrium marginal productivity of labour, which is unity. This fact allows focusing attention on the case in which $p_{t}=w_{t}$ for all $t$, without any loss of generality. 
An agent's intertemporal characteristics may then be described as follows. Consumption $c_{\tau}$ in each period $\tau$ of his life $(\tau=1,2)$ must be nonnegative. On the other hand, it is assumed that the agent has a labour endowment in each period of his life, $\ell_{\tau}^{*}$, and that his labour supply $\ell_{\tau}$, or equivalently his consumption of leisure $\ell_{\tau}^{*}-l_{\tau}$, must satisfy $0 \leqq \ell_{\tau}^{*}-\ell_{\tau} \leqq \ell_{\tau}^{*}$ for $\tau=1,2$. The agent's intertemporal tastes are represented by the utility function

$$
u_{1}\left(c_{1}, \ell_{1}^{*}-\ell_{1}\right)+U_{2}\left(c_{2}, \ell_{2}^{*}-\ell_{2}\right)
$$

which is defined on the set of $c_{\tau}$ and $\ell_{\tau}^{\star}-\ell_{\tau}$ that satisfy the foregoing feasibility constraints. We shall assume

(1.a) $\ell_{1}^{*}>0$ and $\ell_{2}^{*}>0$

(1.b) $U_{\tau}\left(c_{\tau}, l_{\tau}^{*}-l_{\tau}\right)$ is continuous, increasing in each argument and strictly concave for $\tau=1,2$.

We consider now the decision problem that a young agent has to solve at an arbitrary date. Let $p>0$ be the money price of the good that he observes in the current period and let $p^{e}>0$ be the price that he expects for the next date (the reader is reminded that current and expected money wages are taken to be equal to $p$ and $p^{e}$ respectively). The agent's problem is then to choose his current consumption $c_{1}$, his current labour supply $\ell_{1}$, his demand for nominal money $m$, and to plan for the next date his future consumption $c_{2}$ and labour supply $\ell_{2}$. Formaliy, 
choose $c_{1}, \ell_{1}, m, c_{2}, l_{2}$ so as to maximize $U_{1}\left(c_{1}, \ell_{1}^{*}-\ell_{1}\right)+U_{2}\left(c_{2}, \ell_{2}^{\star}-l_{2}\right)$ subject to $c_{\tau} \geqq 0,0 \leqq \ell_{\tau} \leqq \ell_{\tau}^{*} \quad(\tau=1,2), m \geqq 0$ and the current and expected budget constraints

$$
\begin{aligned}
& p\left(c_{1}-\ell_{1}\right)+m=0 \\
& p^{e}\left(c_{2}-\ell_{2}\right)=m
\end{aligned}
$$

It is routine to verify that under assumption $(1.6)$, this problem has a unique solution. It is moreover clear that the optimum values of $\left(c_{\tau}-\ell_{\tau}\right)$ for $\tau=1,2$ are unchanged whenever current and expected prices are changed proportionnally - in other words they depend only on the ratio $\theta=\mathrm{p} / \mathrm{p}^{\mathrm{e}}$, or equivalently on the consumer's expected real interest rate $\rho=\theta-1$ (absence of money illusion). We shall denote them $z_{1}(\theta)$ and $z_{2}(\theta)$, respectively. Since one unit of labour yields one unit of good, we may interpret these values as the trader's current and expected excess demands for the good. On the other hand, the optimum value of $m$ that arises from (1.1) - the trader's demand for money is clearly a function of $p$ ard of $p^{e}$ that is homogenous of degree 1 with respect to these variables. The trader's demand for money $m^{d}\left(p, p^{e}\right)$ is in fact linked to the excess demand functions $z_{1}(\theta)$ and $z_{2}(\theta)$ by the following identities, which are consequences of the budget constraints of (1.1).

$$
p z_{1}(\theta)+m^{d}\left(p, p^{e}\right) \equiv 0
$$

for every $p, p^{e}$ and $\theta=p / p^{e}$. These identities imply of course 


$$
\theta z_{1}(\theta)+z_{2}(\theta) \equiv 0 \text { for every } \theta>0
$$

One may note that the demand for "real balances", $\mathrm{m}^{\mathrm{d}} / \mathrm{p}$ is then given by $-z_{1}(\theta)=z_{2}(\theta) / \theta$.

It is convenient to decompose the decision problem (1.1) into two subproblems. Let us rewrite the budget constraints of (1.1) under the following form

$$
\begin{aligned}
& p\left[c_{1}+\left(\ell_{1}^{*}-\ell_{1}\right)\right]+m=p \ell_{1}^{*} \\
& p^{e}\left[c_{2}+\left(\ell_{2}^{*}-\ell_{2}\right)\right]=p^{e} \ell_{2}^{*}+m
\end{aligned}
$$

Then it is clear that the consumer may solve (1.1) in two steps. First, given an arbitrary $m$ satisfying $0 \leqq m \leqq p \ell_{1}^{*}$, he may choose the consumptions of good and of leisure in each period of his life that maximize each utility function $U_{\tau}$ under either the budget constraint (1.5) when $\tau=1$, or (1.6) when $\tau=2$. Having achieved this, the trader may then choose the optimal level of $\mathrm{m}$. This motivates considering the following problem.

Given $\mathrm{a}_{\tau} \geqq 0$, choose $\mathrm{c}_{\tau}$ and $\ell_{\tau}^{*}-l_{\tau}$, with $\mathrm{c}_{\tau} \geqq 0$, $0 \leqq \ell_{\tau}^{*}-l_{\tau} \leqq \ell_{\tau}^{*}$, so as to maximize $U_{\tau}\left(c_{\tau}, \ell_{\tau}^{*}-\ell_{\tau}\right)$ subject to

$$
c_{\tau}+\left(\ell_{\tau}^{*}-\ell_{\tau}\right)=a_{\tau}
$$

Under assumption $(1.6),(1.7)$ has a unique solution. In fact, this problem determines the agent's optimum consumption $c_{\tau}\left(a_{\tau}\right)$ and his optimum labour supply $\ell_{\tau}\left(a_{\tau}\right)$ in each period of his life as a function of his "real wealth" $a_{\tau}$. These functions are linked by 


$$
c_{\tau}\left(a_{\tau}\right)+\ell_{\tau}^{*}-\ell_{\tau}\left(a_{\tau}\right) \equiv a_{\tau} \quad \text { for every } a_{\tau} \geq 0 \text {. }
$$

Let $V_{\tau}\left(a_{\tau}\right)$ be the maximum of $U_{\tau}$ that is obtained in (1.7). It is quite easy to verify that under assumption $(1.6), V_{\tau}$ is continuous, increasing, strictly quasiconcave. Let us consider finally

$$
\text { Given } p>0, p^{e}>0 \text {, choose } a_{1} \geqq 0, a_{2} \geqq 0 \text { and } m \geqq 0
$$
so as to maximize $v_{1}\left(a_{1}\right)+v_{2}\left(a_{2}\right)$ subject to

$$
\begin{aligned}
& p a_{1}+m=p \ell_{1}^{*} \\
& p e^{e}=p \ell_{2}^{e}+m
\end{aligned}
$$

By comparing the budget constraints of (1.9) with (1.5) and (1.6), it is clear that the optimum values of $a_{1}-l_{1}^{*}$, of $a_{2}-l_{2}^{*}$ and of $m$ that result from (1.9) coincide indeed with $z_{1}(\theta), z_{2}(\theta)$ and $m^{d}\left(p, p^{e}\right)$, respectively.

Note that problems (1.7) and (1.9) admit a very simple graphical representation as shown in Fig. 1.a and Fig. 1.b. The first Figure is a direct description of (1.7) and need no comment. Fig. 1.b, which represents (1.9), is drawn in the plane $\left(a_{1}, a_{2}\right)$. The two constraints become there the intertemporal budget constraint

$$
\theta a_{1}+a_{2}=\theta l_{1}^{*}+\ell_{2}^{*}
$$

which is obtained by adding the two budget constraints of (1.9) and by dividing the result by the expected price $p^{e}$, and the liquidity constraint $a_{1} \leqq \ell_{1}^{*}$ which expresses the fact that money balances 
cannot be negative.

Fig. 1.a

Fig. 1.6

The main advantage of going through (1.7) and (1.9) is that such a procedure will enable us to state our assumptions compactly and more transparently by using the indirect utility functions $V_{\tau}\left(a_{\tau}\right)$ instead of deriving them from the original functions $U_{\tau}$ - which would have been in some cases quite tedious.

We have seen that under assumption (1.b), each indirect utility function $V_{\tau}$ is continuous, strictly concave and increasing. We shall make in fact the stronger assumption :

(1.c) For each $\tau=1,2$, the indirect utility function $V_{\tau}$ is continuous on $[0,+\infty)$ and twice continuously differentiable on $(0,+\infty)$, with $V_{\tau}^{\prime}\left(a_{\tau}\right)>0$ $\lim _{a_{\tau} \rightarrow 0} V_{\tau}^{\prime}\left(a_{\tau}\right)=+\infty, V_{\tau}^{\prime \prime}\left(a_{\tau}\right)<0$

$\overline{1}$

Going through (1.7) and (1.9) shows incidentally that considering a variable production model like this one is formally very similar to looking at an exchange economy (i.e. without production) in which every agent is endowed with the quantity $\ell_{\tau}^{*}$ of the good in each period of his tife.

It is possible to derive such differentiability properties under appropriate (differentiability) assumptions involving the original utility functions $U_{\tau}$ only, provided that "corner" solutions are prevented to occur in (1.7). Such is the case for example if each $U_{\tau}$ is continuously differentiable and if the marginal rate of substitution between consumption and leisure, $U_{\tau c}^{\prime} / U_{\tau, l^{*}-l}^{\prime}$, is equal to 0 whenever $c_{\tau}>0, \ell_{\tau}^{*}-\ell_{\tau}=0$, and to $+\infty$ whenever $c_{\tau}=0, \ell_{\tau}^{*}-l_{\tau}>0$ or whenever $\ell_{\tau}^{*}=l_{\tau}-l_{\tau}^{*}$ (see Fig.1.a). 

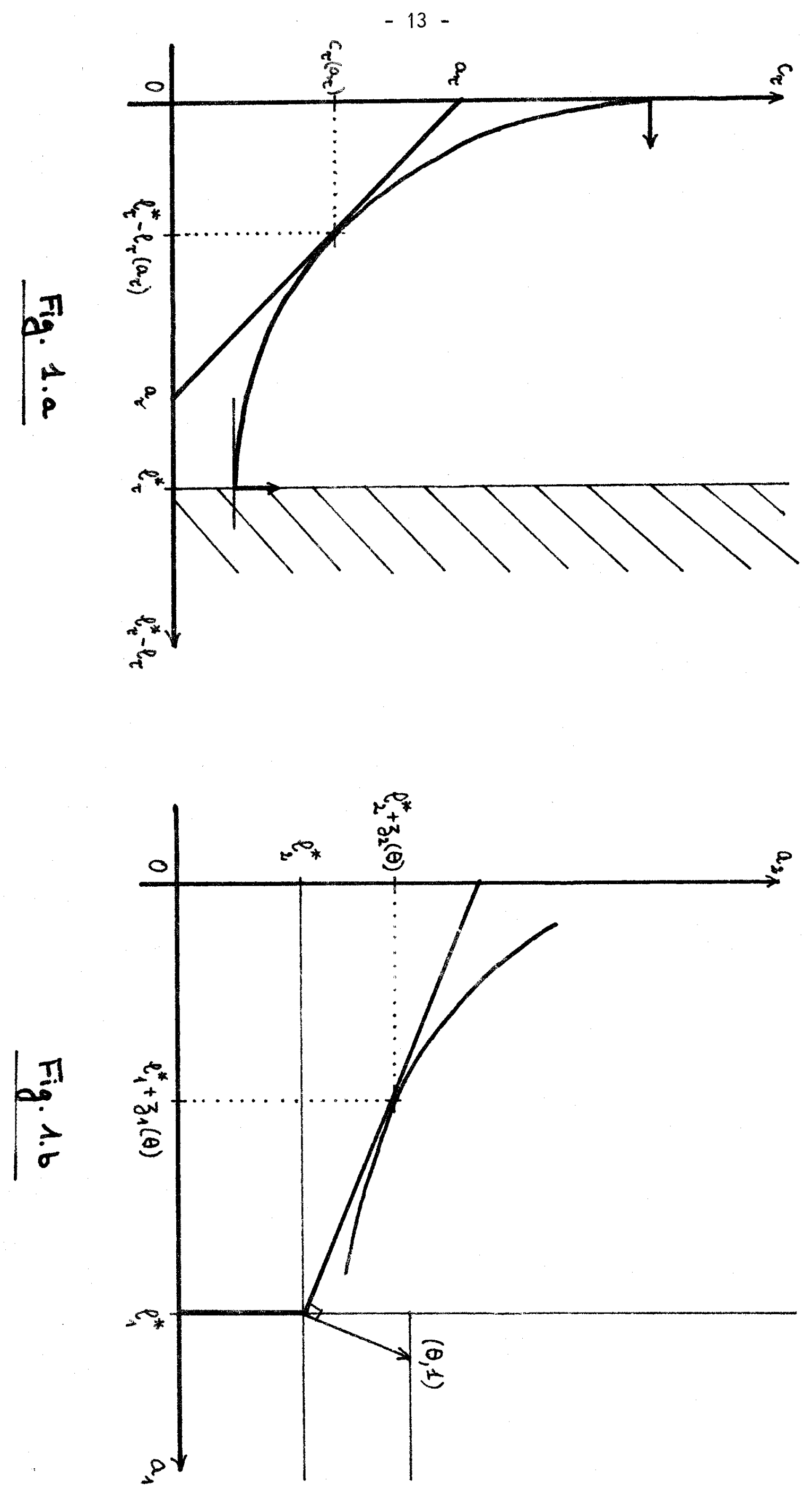
We end up this section with a brief analysis of a few elementary facts about the excess demand functions $z_{1}$ and $z_{2}$ that will be used repeatedly in the sequel.

LEMMA 1.1. Assume (1.a) and (1.c) and let $\bar{\theta}=V_{1}^{\prime}\left(l_{1}^{*}\right) / V_{2}^{\prime}\left(l_{2}^{*}\right)$. Then $z_{1}(\theta)$ and $z_{2}(\theta)$ are continuous on the open interval $(0,+\infty)$. Moreover,

1) $z_{1}(\theta)=z_{2}(\theta)=0$ whenever $\theta \leqq \bar{\theta}$, and $-\ell_{1}^{*}<z_{1}(\theta)<0$, $z_{2}(\theta)>0$ whenever $\theta>\bar{\theta}$

2) for every $\theta \geqq \bar{\theta}$, one has

$$
V_{1}^{\prime}\left(\ell_{1}^{*}+z_{1}(\theta)\right)=\theta V_{2}^{\prime}\left(\ell_{2}^{*}+z_{2}(\theta)\right)
$$

We shall not give a formal proof of this statement, which follows from elementary considerations, but rather give the intuition behind it by looking at Fig. 1.b. The parameter $\bar{\theta}$ is indeed the inverse of the marginal rate of substitution $V_{2}^{\prime}\left(a_{2}\right) / V_{1}\left(a_{1}\right)$ at the endowment point $\left(\ell_{1}^{*}, \ell_{2}^{*}\right)$. If $\theta \leqq \bar{\theta}$, the liquidity constraint $a_{1} \leqq \ell_{1}^{*}$ is binding. The demand for money then vanishes and $z_{1}(\theta)=z_{2}(\theta)=0$. If $\theta>\bar{\theta}$, the liquidity constraint is no longer binding - the demand for money is positive in which case $z_{1}(\theta)<0$ and $z_{2}(\theta)>0$, and the optimum of (1.9) requires $a_{1}>0$ (since $\left.V_{1}(0)=+\infty\right)$, thus $z_{1}(\theta)>-l_{1}^{*}$. Therefore at the optimum of $(1.9)$, one must have $V_{1}^{\prime}\left(a_{1}\right)=\theta V_{2}^{\prime}\left(a_{2}\right)$, hence $(1,10)$. Note that in view of (1.4), the relation (1.10) may take the equivalent form

$$
-z_{1}(\theta) V_{1}\left(\ell_{1}^{*}+z_{1}(\theta)\right)=z_{2}(\theta) V_{2}^{\prime}\left(\ell_{2}^{*}+z_{2}(\theta)\right) \text { for every } \theta \geqq \bar{\theta}
$$


This brief argument implies that a consumer will have a positive demand for money if and onty if $\theta>\bar{\theta}$. We shall be concerned in the sequel with monetary competitive equilibria, where the agents have to hold the outstanding money stock, which is positive. In order to ensure the existence of such equilibria, it will be particularly important that the traders have enough incentives to save when the price of the good is constant (and is expected to be constant) over time. This case corresponds to $\mathrm{p}^{\mathrm{e}}=\mathrm{p}$ and thus to $\theta=1$. We shall need accordingly that $1>\bar{\theta}$. Forma11y,

(1.d) $\bar{\theta}=V_{1}^{\prime}\left(\ell_{1}^{*}\right) / V_{2}^{\prime}\left(\ell_{2}^{*}\right)$ is less than 1 .

The next fact gives more information about the variation of $z_{1}$ and $z_{2}$ with $\theta$

LEMMA 1.2. Assume (1.a) and (1.c). Then the restrictions of the excess demand functions $z_{1}(\theta)$ and $z_{2}(\theta)$ to the interval $[\bar{\theta},+\infty)$ are continuousey differentiable. For every $\theta \geqq \bar{\theta}$

$$
\begin{aligned}
& z_{1}^{\prime}(\theta)=\left[V_{2}^{\prime}\left(\ell_{2}^{*}+z_{2}(\theta)\right)+z_{2}(\theta) V_{2}^{\prime \prime}\left(\ell_{2}^{*}+z_{2}(\theta)\right)\right] / \Delta \\
& z_{2}^{\prime}(\theta)=-\left[V_{1}^{\prime}\left(\ell_{1}^{*}+z_{1}(\theta)\right)+z_{1}(\theta) V_{1}^{\prime \prime}\left(\ell_{1}^{*}+z_{1}(\theta)\right)\right] / \Delta
\end{aligned}
$$

in which $\Delta=V_{1}^{\prime \prime}\left(\ell_{1}^{*}+z_{1}(\theta)\right)+\theta^{2} V_{2}^{\prime \prime}\left(\ell_{2}^{*}+z_{2}(\theta)\right)<0$. In particular $z_{2}^{\prime}(\theta)>0$ for every $\theta \geqq \bar{\theta}$. Moreover $z_{2}(\theta)$ diverges to to whenever $\theta$ tends to +o . 
The first part of this statement is obvious by differentiating (1.10) or (1.11) and by using (1.4). On the other hand, the fact that $\mathrm{z}_{2}(\theta)$ diverges to to whenever $\theta$ increases without bound is not difficult to verify. Indeed, we get from (1.4) that when $\theta$ tends to $+\infty, z_{1}(\theta)$ must tend to 0 if $z_{2}(\theta)$ remains bounded. But in that case, the left hand side of $(1.10)$ is bounded while the right hand side diverges to $+\infty$. This is a contradiction which shows that $\lim _{\theta \rightarrow+\infty} z_{2}(\theta)=+\infty$.

The foregoing analysis gives some insight about the consequences upon a trader's behaviour of a variation of $\theta$ (or of his "expected real interest rate" which is given by $\theta-1$ ). A change of $\theta$ generates an intertemporal substitution - through the variation of the relative price of future and current consumption - as well as income or wealth effects - in particular because intertemporal income in (1.9) is equal to $\theta l_{1}^{*}+l_{2}^{*}$. Lemma 1.2 states that a rise of $\theta$ induces always an increase of $z_{2}(\theta)$. Loosely speaking, this is because intertemporal substitution and income effects do work then in the same direction. On the other hand, the induced variation of $z_{1}(\theta)$ is ambiguous. We know indeed from Lemma 2 that $-z_{2}^{\prime}(\bar{\theta}) / z_{1}^{\prime}(\bar{\theta})=\bar{\theta}$. Therefore $z_{1}^{\prime}(\bar{\theta})<0$, and thus by continuity $z_{1}^{\prime}(\theta)<0$ if $\theta$ is larger than but close enough to $\bar{\theta}$. However, the sign of $z_{j}^{\prime}(\theta)$ is a priori indeterminate for large values of $\theta$, because income and substitution effects are working in opposite directions in such a case. As a matter of fact the origin of the business cycles that are going to be analysed in the present paper is precisely this potential conflict between intertemporal substitution and wealth effects. 
Examination of the expressions of $z_{1}^{\prime}(\theta)$ and $z_{2}^{\prime}(\theta)$ suggests that an important role in this regard should be played by the so-called Arrow-Pratt relative degrees of risk aversion

$$
R_{\tau}\left(a_{\tau}\right)=-V_{\tau}^{\prime \prime}\left(a_{\tau}\right) a_{\tau} / V_{\tau}^{\prime}\left(a_{\tau}\right)
$$

which are well defined whenever $a_{\tau}>0$. These expressions measure in effect the degree of concavity (curvature) of each $V_{\tau}$. We shall stick throughout to the usual terminology and speak of "risk aversion", although it is slightly ackward in the present context, which involves no uncertainty. We shall use the following assumption

(1.e) $\quad R_{2}\left(a_{2}\right)$ is a nondecreasing function of $a_{2}$ for every $a_{2}>0$.

For a justification of such an assumption (in a context involving uncertainty) see Arrow (1970, Ch. 3). It is then easy to get the following fact.

LEMMA 1.3. Assume (1.a) and (1.c). Then for every $\theta \geqslant \bar{\theta}, z_{1}^{\prime}(\theta)<0$ if and only if $R_{2}\left(\ell_{2}^{*}+z_{2}(\theta)\right)<\left(\ell_{2}^{*}+z_{2}(\theta)\right) / z_{2}(\theta)$. Accordingly,

1) if $R_{2}\left(a_{2}\right) \leqq 1$ for all $a_{2}>0$, then $z_{1}^{\prime}(\theta)<0$ for every

$\theta \geqq \bar{\theta}$

2) if (1.e) holds and if there exists $a_{2}>0$ such that $R_{2}\left(a_{2}\right)>1$, then there exists a unique $\theta^{*}>\bar{\theta}$ such that $z_{1}^{\prime}(\theta)<0$ for every $\bar{\theta} \leqq \theta<\theta^{*}, z_{1}^{\prime}\left(\theta^{*}\right)=0$ and $z_{1}^{\prime}(\theta)>0$ for every $\theta>\theta^{*}$.

The claim that $z_{1}^{\prime}(\theta)<0$ if and only if $R_{2}\left(l_{2}^{*}+z_{2}(\theta)\right)<\left(\ell_{2}^{*}+z_{2}(\theta)\right) / z_{2}(\theta)$ is immediate to verify by looking at the expression of $z_{1}(\theta)$ in Lemma 1.2. Then if $R_{2}\left(a_{2}\right) \leqq 1$ for all $a_{2}>0$, the left hand side of this inequality never exceeds 1 , 
while the right hand side is always greater than 1 whenever $\theta \geqq \bar{\theta}$, which shows 1). If (1.e) holds, the left hand side of this inequality is a non-decreasing function of $\theta$. When $\theta$ tends to to, $z_{2}(\theta)$ tends also to to and thus under the assumptions of 2$), R_{2}\left(\ell_{2}^{*}+z_{2}(\theta)\right)$ exceeds 1 for $\theta$ large enough. On the other hand, $\left(\ell_{2}^{*}+z_{2}(\theta)\right) / z_{2}(\theta)$ decreases from to to 1 when $\theta$ rises from $\bar{\theta}$ to to. There is thus, by continuity, a unique $\theta^{*}>\bar{\theta}$ such that

$$
R_{2}\left(\ell_{2}^{*}+z_{2}\left(\theta^{*}\right)\right)=\left(\ell_{2}^{*}+z_{2}\left(\theta^{*}\right)\right) / z_{2}\left(\theta^{*}\right)
$$

and it is clear that $z_{1}^{\prime}(\theta)<0$ whenever $\bar{\theta} \leqq \theta<\theta^{*}, z_{1}^{\prime}\left(\theta^{*}\right)=0$ and $z_{1}^{\prime}(\theta)>0$ when $\theta>\theta^{*}$.

It may be useful to illustrate our findings by drawing in the plane $\left(a_{1}, a_{2}\right)$ a consumer's offer curve, that is, the locus of all points of coordinates $a_{1}=\ell_{1}^{*}+z_{1}(\theta), a_{2}=\ell_{2}^{*}+z_{2}(\theta)$ when $\theta$ varies. The result is shown in Fig. 2 below. According to the previous lemmas, the offer curve is smooth and goes through the endowment point $A=\left(\ell_{1}^{*}, \ell_{2}^{*}\right)$ - this corresponds to $\theta \leqq \bar{\theta}$. Its normal there is the vector $(\bar{\theta}, 1)$. The curve 7 ies below the $45^{\circ}$ line $A B$ when $\bar{\theta}<\theta<1$, and above when $\theta>1$. Figures 2.a and 2.b are drawn under the assumption that $\bar{\theta}<1$. Of course, if $\bar{\theta} \geqq 1$, the curve would lie entirely above AB. Fig. 1.a corresponds to the case considered in 1 ) of Lemma 1.3 , in which $R_{2}\left(a_{2}\right) \leqq 1$ for all $a_{2}>1$. The curve is then "monotone", i.e. it has no critical point. Fig. 1.b corresponds to the case in which (1.e) holds and in which $R_{2}\left(a_{2}\right)>1$ for some $a_{2}>0$. The offer curve has then a unique critical point corresponding to the value $\theta=\theta^{*}$. 

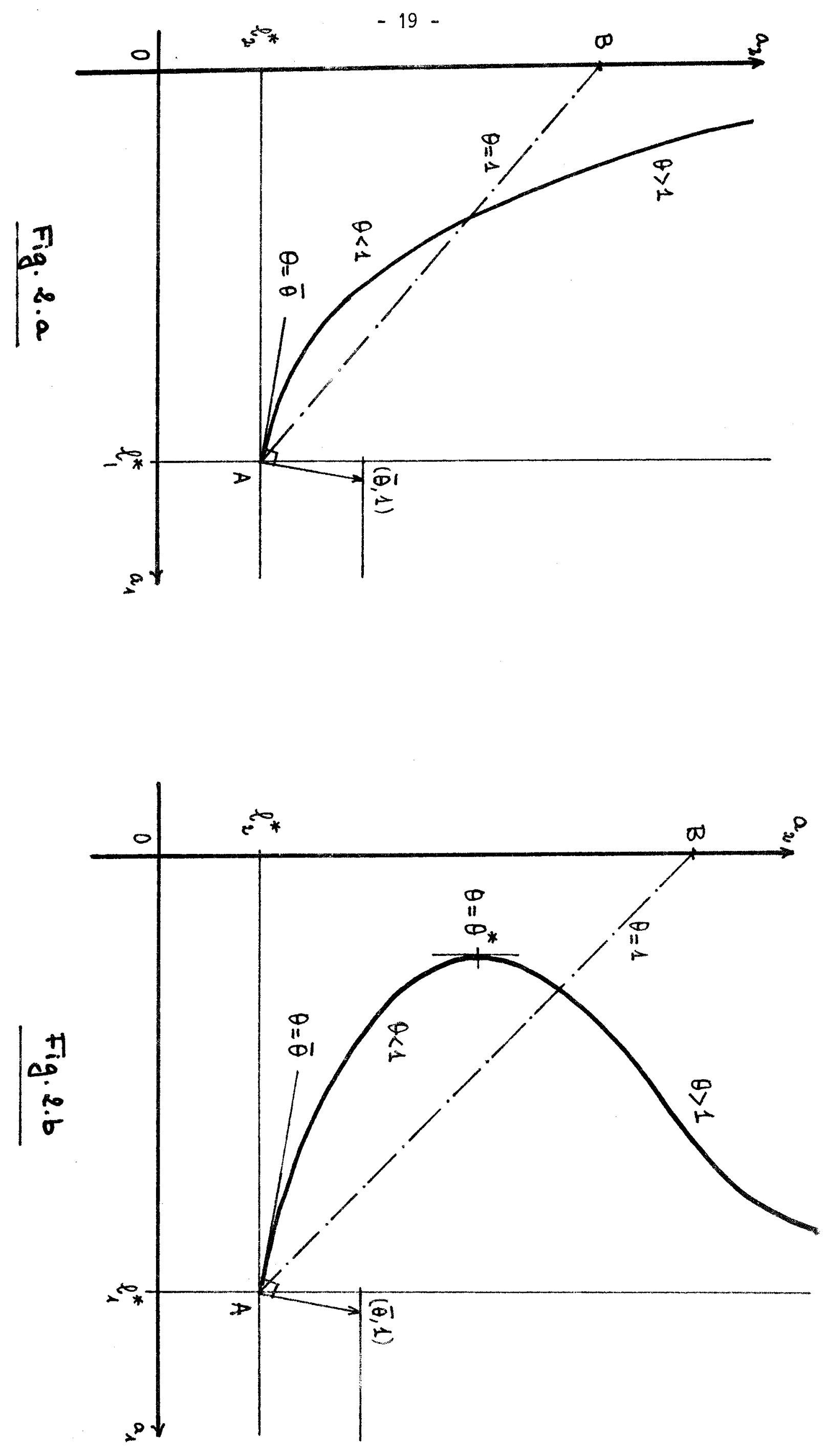
Remark. The preceding lemmas have been stated by employing the excess demand functions $z_{1}$ and $z_{2}$. The implications of these results on the demand for real balances

$$
m^{d}\left(p, p^{e}\right) / p=z_{2}(\theta) / \theta \equiv-z_{1}(\theta)
$$

are obvious. Finally, note that the elasticity of the excess demand function $z_{2}$, i.e. $\theta z_{2}^{\prime}(\theta) / z_{2}(\theta)$, is greater than 1 if and only if $z_{1}^{\prime}(\theta)>0$. 


\section{PERIODIC COMPETITIVE EQUILIBRIA.}

The preceding Section was devoted to rather elementary and standard considerations about the "microeconomic" behaviour of a consumer. We turn now attention to the study of the dynamics of the economy and to the definition of periodic equilibria.

Since there are competitive spot markets for output, labour and money at every date, the evolution of the economy is described by a sequence of temporary competitive equilibria. Agents are assumed to forecast at every date future prices through a given learning process, during the adjustment of the economy toward "steady states" - which will mean here periodic sequences of temporary competitive equilibria. It will be postulated however that along such periodic sequences, traders have "rational" expectations, i.e., that they forecast correctly the periodic sequence of prices. This way of proceeding comes from the fact that learning processes belong in our view to the important characteristics of the traders on the same level as preferences, endowments and the like, and that perfect foresight must be modelled as a property of those learning processes in relationship to specific environments. In particular, perfect foresight appears to be a plausible outcome of learning when a trader's environment is repetitive enough (here periodic), but seems to be far less acceptable out of such special circumstances. An other reason that justifies our approach is that we shall study later on the stability of a long run periodic equilibrium, and that for that purpose, taking into account that traders are learning on the transition path is important.

Let us consider the economy at some date $t$. We look first at the "old" consumer living at $t$. This consumer holds the outstanding stock of money $M$. If the price quoted at $t$ is $p_{t}>0$, his problem is then to maximize $U\left(c, \ell_{2}^{*}-\ell\right)$ subject to $c \geqq 0,0 \leqq \ell_{2}^{*}-\ell \leqq \ell_{2}^{\star}$ and 


$$
p_{t}\left[c+\left(\ell_{2}^{*}-\ell\right)\right]=p_{t} \ell_{2}^{*}+M
$$

(we have taken advantage here again of the fact that the nominal wage rate $w_{t}$ must be equal to $p_{t}$ in equilibrium). In view of the problem (1.7), the result will be $c_{2}\left(\ell_{2}^{*}+M / p_{t}\right)$ and $\left.\ell_{2}\left(\ell_{2}^{*}+M / p_{t}\right)\right)$. But the important point to note at this stage is that, since one unit of labour yields one unit of output, the old consumer's excess demand for the good may be described by $c-\ell$, and that this quantity is equal to the real value of his money stock $M / p_{t}$.

Consider next the "young" consumer living at $t$. This consumer observes the current price $p_{t}$, and we assume that he knows past prices. To simplify, we shall postulate that the consumer processes only the current price $p_{t}$ and the past (positive) prices $p_{t-1}, \ldots, p_{t-T}$ to compute the (positive) price $p_{t+1}^{e}$ that he expects to prevail at the next date. In what follows, we shall think of $T$ as finite, but "large". We describe this "learning" process - which may be a simple rule of thumb or a complicated statistical technique - by an expectation function

$$
p_{t+1}^{e}=\psi\left(p_{t}, p_{t-1}, \ldots, p_{t-T}\right)
$$

We assume that the $\operatorname{lag} T$ as well as the function $\psi$ are independent of time. 'Thus, in view of the analysis of the preceding section, the young trader's excess demand for the good is given by $z_{1}\left(p_{t} / \psi\left(p_{t}, p_{t-1}, \ldots, p_{t-T}\right)\right)$ while his demand for money is $m^{d}\left(p_{t}, \psi\left(p_{t}, p_{t-1}, \ldots, p_{t-T}\right)\right)$.

1 The assumption that a trader's information about the past is composed only of prices is innessential. The arguments of this paper all go through without any alteration if young traders were informed of past quantities as we11. 
A temporary competitive equilibrium at $t$ is then defined as a price $p_{t}>0$ such that all markets clear at that date. For the good market, this yields

$$
z_{1}\left(p_{t} / \psi\left(p_{t}, p_{t-1}, \ldots, p_{t-T}\right)\right)+\left(M / p_{t}\right)=0
$$

As for money, we get

$$
m^{d}\left(p_{t}, \psi\left(p_{t}, p_{t-1}, \ldots, p_{t-T}\right)\right)=M
$$

Of course, in view of the young trader's current budget constraint (1.2), the two equations are equivalent. This is in fact Walras's Law $^{1}$.

The next assumption on the expectation function is made to guarantee the existence of a solution $p_{t}$ to the above system of equations, when past prices are given. It is there essentially to ensure enough intertemporal substitution when the current price $p_{t}$ varies.

(2.1) The expectation function $\psi$ is continuously differentiable. The elasticity of $\psi$ with respect to the current price is between 0 and 1 , that is ${ }^{2}$

$$
0 \leqq \psi_{0}^{\prime}\left(p_{t}, p_{t-1}, \ldots, p_{t-T}\right) p_{t} / \psi\left(p_{t}, p_{t-1}, \ldots, p_{t-T}\right) \leqq 1
$$

Moreover, the ratio $\psi\left(p_{t}, \ldots, p_{t-T}\right) / p_{t}$ tends to 0 when $p_{t}$ goes to $+\infty$.

We have not to write down of course the equilibrium condition for labour since it has been al ready taken into account by assuming that $w_{t}=p_{t}$ and that output was equal to the traders' labour supply. 2 The notation $\psi_{j}^{\prime}\left(p_{t}, \ldots, p_{t-T}\right)$ stands for the partial derivative of $\psi$ with respect to $p_{t-j}$ for $j=0,1, \ldots, T$. 
We may then state

PROPOSITION 2.1. Assume (1.a) and (1.c). Then under assumption (2. b), there exists a unique temporary competitive equilibrium price $\mathrm{p}_{t}$, given the past prices $p_{t-1}, \ldots, p_{t-T}$. The temporary walrasian equilibrium function so defined $p_{t}=W\left(p_{t-1}, \ldots, p_{t-T}\right)$ is continuously differentiable.

The proof of this claim is simple. By virtue of Walras's Law, we may focus attention on the good market equation (2.1) alone. Then when $p_{t}$ toes to 0 , the real balance $M / p_{t}$ goes to infinity while the young consumer's excess demand for the good is bounded below by $-\ell_{1}^{*}$. Thus we are sure that aggregate excess demand goes to to on the good market when $p_{t}$ decreases to 0 . This is due to the real balance effect, which operates on the old trader alone.

We wish now to show that an excess supply of the good appears when its price increases without bound. Or equivalently - again by Walras's Law - that an excess demand for money appears in such a circumstance. But that is easy if one considers the money equation (2.2) which can be put in the following equivalent but more convenient form by using the young trader's expected budget constraint (1.3)

$$
\psi\left(p_{t}, \ldots, p_{t-T}\right) z_{2}\left(p_{t} / \psi\left(p_{t}, \ldots, p_{t-T}\right)\right)=M
$$

Under assumption $\left(2 .\{), \psi\right.$ and $\mathrm{p}_{\mathrm{t}} / \psi$ are nondecreasing functions of $\mathrm{p}_{\mathrm{t}}$. Moreover $p_{t} / \psi$ tends to to when $p_{t}$ increases without bound. In view of Lemma 1.2, the left hand side of (2.3), that is, the young trader's demand for money, is nondecreasing and goes to +o (and thus exceeds the money supply M) when $p_{t}$ tends to to, which proves our claim. Here, it is the 
intertemporal substitution effect that acts upon the young trader which plays a central role ${ }^{1}$.

We may therefore conclude that by continuity, (2.1) admits a solution. Unicity follows readily by considering $(2.3)$, which is equivalent, as we said, to (2.1). We showed that the left hand side of (2.3) is nondecreasing. This implies either that it is positive for all $\mathrm{p}_{t}$, or that it is equal to 0 whenever $p_{t} \leqq \bar{p}_{t}$ for some $\bar{p}_{t}>0$ and positive otherwise. When it is positive, we may meaningfully consider its partial derivative with respect to $\mathrm{p}_{\mathrm{t}}$, which is given by

$$
\psi_{0}^{\prime} z_{2}+z_{2}^{\prime}\left(1-\frac{p_{t} \psi_{0}^{\prime}}{\psi}\right)
$$

(we have suppressed here the arguments of the functions involved for notational simplicity) and which is positive under assumption (2.6). The left hand side of (2.3) is therefore increasing whenever it is positive, which proves uniqueness. Continuous differentiability of the temporary Walrasian equilibrium function $W$ is then a consequence of the implicit function theorem by the same sort of argument. Partial derivatives of $W$ can be easily computed for instance by differentiating $(2.3)^{2}$

For a further discussion of the respective roles of wealth and intertemporal substitution effects in a competitive monetary economy see

2 Grandmont (1983a).

The fact that the equilibrium price $p_{t}$ at $t$ depends only on past prices is due the simplified structure of the model. For instance, if there were several different consumers, the current equilibrium would depend also on the initial distribution of money holdings. If utilities were not separable over time, it would depend also on past consumptions. In general, current equilibrium prices would depend on past equilibrium states (prices, consumptions, money holdings and so on). See, e.g. Grandmont and Hildenbrand (1974), Grandmont and Laroque (1973). 
It may be noted in addition that under the assumptions of the Proposition, the unique temporary equilibrium price $p_{t}$ is stable in any Walrasian tatonnement process at date $t$ in which prices respond positively to excess demand.

We proceed now to the definition of periodic competitive equilibria.

A periodic competitive equilibrium with (primitive) period $k$ is a sequence of temporary equilibria, that is, an infinite sequence of positive prices $\left(p_{t}\right)$ that satisfies (2.1) and (2.2) for all $t$, such that $p_{t+k}=p_{t}$ for all $t$ and such that $k$ is the smallest period of the sequence $\left(i . e\right.$. there is no $k^{\prime}<k$ such that $p_{t+k^{\prime}}=p_{t}$ for all $t$ ).

On gets of course as a particular case a stationary equilibrium when $k=1$. We may and we shall often identify a periodic competitive equilibrium with the orbit of the periodic sequence $\left(p_{t}\right)$, i.e. with the $k$ consecutive values $\left(p_{1}^{*}, \ldots, p_{k}^{*}\right)$ that $\left(p_{t}\right)$ takes. This identification supposes of course that the two orbits $\left(p_{1}^{*}, \ldots, p_{k}^{*}\right)$ and $\left(p_{j}^{*}, \ldots, p_{k}^{*}, p_{1}^{*}, \ldots, p_{i-1}\right)$ are considered as equivalent.

Note that the above definition involves only the functions $z_{1}, z_{2}, m^{d}$ and $\psi$ which describe a trader's behaviour - it does not use assumption (2.6). If (2.6) is postulated, the temporary equilibrium map $W$ is well defined and one may view a periodic competitive equilibrium as a periodic solution of

$$
p_{t}=w\left(p_{t-1}, \ldots, p_{t-T}\right)
$$

In fact, it is more convenient to look at (2.4) as defining a dynamic system in a space of larger dimension. For any $q_{t-1}=\left(p_{t-1}, \ldots, p_{t-T}\right)$, let us consider $q_{t}=\tilde{W}\left(q_{t-1}\right)$ that is determined by

$$
q_{t}=\left(w\left(p_{t-1}, \ldots, p_{t-T}\right), p_{t-1}, \ldots, p_{t-T+1}\right)
$$


This procedure defines a function $\tilde{W}$ that takes the interior of $\mathbb{R}_{+}^{\top}$ into itself, and it is clear that the equation $q_{t}=\tilde{W}\left(q_{t-1}\right)$ describes the same dynamics as $(2.4)$ through the relation $q_{t-1}=\left(p_{t-1}, \ldots, p_{t-T}\right)$ for all t .

We may in particular state for later reference ${ }^{1}$

LEMMA 2.2. Assume $(1, a),(1, c)$ and $(2.6)$. Let $\left(p_{t}\right)$ be a periodic sequence of positive prices with period $k$ and let $\left(p_{1}^{*}, \ldots, p_{k}^{*}\right)$ be its orbit. Let us define for all $i=1, \ldots, k$

$$
q_{i}^{*}=\left(p_{i}^{*}, \ldots, p_{1}^{*}, p_{k}^{*}, \ldots, p_{1}^{*}, \ldots\right)
$$

in which $q_{i}^{*}$ is a vector of $\mathbb{R}^{\top}$. Then $\left(p_{1}^{*}, \ldots, p_{k}^{*}\right)$ is a periodic competitive equilibrium if and only if $\left(q_{1}^{*}, \ldots, q_{k}^{*}\right)$ is a periodic orbit of $\tilde{W}$ with period $k$.

As such however, the concept is not very interesting, for it permits that traders still make forecasting errors even though their environment is repetitive enough to enable them to discover the laws governing this regularity. The next assumption states that the traders may "learn" and thus make mistakes when their environment is chaotic, but that they are clever enough to recognize that prices are periodic.

The iterates of a function $f$ of a set $x$ into itself are defined recursively by $f^{1}(x)=f(x), f^{i}(x)=f\left(f^{i-1}(x)\right)$. A periodic orbit (or a cycle) of (primitive) period $k$ of the map $f$ is then defined by $\left(x_{1}^{*}, \ldots, x_{k}^{*}\right)$ such that 1) $x_{1}^{*}$ is a fixed point of $f^{k}$, i.e. $x_{1}^{*}=f^{k}\left(x_{1}^{*}\right)$, and 2) $x_{i}^{*}=f^{i-1}\left(x_{1}^{*}\right) \neq x_{1}^{*}$ for all $i=2, \ldots, k$. This definition implies of course that all $x_{j}^{*}$ of the orbit are fixed points of $f^{k}$, and that they al1 differ. In particular $\left(x_{j}^{*}, \ldots, x_{k}^{*}, x_{1}^{*}, \ldots, x_{i-1}^{*}\right)$ defines the same periodic orbit. 
12.g) The expectation function $\psi$ is consistent with periodicity $k$. More precisely, for any sequence $p_{t}, p_{t-1}, \ldots, p_{t-T}$ that displays period $k$, that is, such that $p_{t-j}=p_{t-j-k}$ for $j=0,1, \ldots$,

$$
\psi\left(p_{t}, \ldots, p_{t-T}\right)=p_{t-k+1}
$$

This assumption implies that agents make correct forecasts along a periodic competitive equilibrium with period $k$. We shall then speak of a periodic competitive equilibrium with perfect foresight. We get then the following very simple characterization

PROPOSITION 2.3. Assume (1.a) and (1.c), and consider an infinite sequence of positive prices $\left(p_{t}\right)$ that displays period $k$. Under assumption $(2 . g)$, $\left(p_{t}\right)$ is a sequence of temporary competitive equilibria if and only if it satisfies for all $t$.

$$
z_{1}\left(\theta_{t}\right)+z_{2}\left(\theta_{t-1}\right)=0
$$

$$
m^{d}\left(p_{t}, p_{t+1}\right) \equiv p_{t+1} z_{2}\left(\theta_{t}\right)=M
$$

in which $\theta_{t}=p_{t} / p_{t+1}$. One has then $\bar{\theta}<\theta_{t}<\overline{\bar{\theta}}=z_{2}^{-1}\left(l_{1}^{*}\right)$ for all $t$.

The statement is in fact immediate. Consider an infinite sequence of positive prices $\left(p_{t}\right)$ with period $k$. Then under assumption (2.g) $\left(p_{t}\right)$ is a sequence of temporary competitive equilibria if and only if it satisfies (2.1) and (2.2), or equivalently (2.3), in which $\psi\left(p_{t}, \ldots, p_{t-T}\right)=p_{t+1}$ for al1 $t$. This yields for a $11 t$ 


$$
z_{1}\left(\theta_{t}\right)+\left(M / p_{t}\right)=0
$$

$$
m^{d}\left(p_{t}, p_{t+1}\right) \equiv p_{t+1} z_{2}\left(\theta_{t}\right)=M
$$

in which $\theta_{t}=p_{t} / p_{t+1}$. Then (2.9) is nothing else than (2.7). Moreover, this relation tells us that an old trader's excess demand for the good at an arbitrary date $\mathrm{t}, \mathrm{M} / \mathrm{p}_{\mathrm{t}}$, is what he planned to do when he was young, i.e. $z_{2}\left(\theta_{t-1}\right)$. Replacing $M / p_{t}$ by $z_{2}\left(\theta_{t-1}\right)$ in (2.8) yields $(2.6)$. The fact that $M$ is positive implies $z_{2}\left(\theta_{t}\right)>0$ and thus $\theta_{t}>\bar{\theta}$. One has then $z_{2}\left(\theta_{t}\right)=-z_{1}\left(\theta_{t}\right)<\ell_{1}^{*}$, and thus $\theta_{t}<\overline{\bar{\theta}}=z_{2}^{-1}\left(\ell_{1}^{*}\right)$.

The system (2.6), (2.7) states simply that markets clear and that consumers forecast correctly future prices at every date ${ }^{1}$. The important property of the system is that it dichotomizes. The good market equation (2.6) determines the periodic sequence of $\theta_{t}$ - or equivalently of real interest rates $\rho_{t}=\theta_{t}-1-$ and indeed a 11 real equilibrium quantities (consumption, output, real balances $M / p_{t}$ ) independently of (2.7), i.e., of the level of the money stock $M$. The level of prices is then in turn determined by the money equation. As a matter of fact, given a sequence $\theta_{t}>\bar{\theta}$ satisfying (2.6) for all $t$, it suffices to fulfill (2.7) at some specific date to guarantee that the whole sequence of prices defined by $p_{t+1}=p_{t} / \theta_{t}$ satisfies the money equation at all dates. Indeed, if (2.7) is fulfilled at date $t-1$, then according to (2.6)

$$
M=p_{t} z_{2}\left(\theta_{t-1}\right)=-p_{t} z_{1}\left(\theta_{t}\right)
$$

That sort of system has been used by numerous writers when studying the dynamics of the overlapping generation model with perfect foresight. See e.g., Azariadis (1981), Cass (1980), Benhabib and Day (1982), Gale (1973). The same sort of approach has been used in Grandmont (1983a) to study monetary steady states in a model involving seyeral goods. 
and thus from (1.4)

$$
M=p_{t} z_{2}\left(\theta_{t}\right) / \theta_{t}=p_{t+1} z_{2}\left(\theta_{t}\right)
$$

Given the equilibrium sequence $\left(\theta_{t}\right)$, the level of corresponding equilibrium money prices is proportional to $M$. A once-for-all change of the level of the money stock is thus neutral in the long run. This is the traditional Quantity Theory.

It may be noted that under assumptions (1.a) and (1.c), the restriction to the interval $[\bar{\theta},+\infty)$ of the excess demand function $z_{2}(\theta)$ is increasing (Lemma 1.2) and therefore has an inverse. Then (2.6) gives rise to a very simple difference equation of the form

$$
\theta_{t-1}=z_{2}^{-1}\left(-z_{1}\left(\theta_{t}\right)\right) \equiv \varphi\left(\theta_{t}\right)
$$

in which the function $\varphi=z_{2}^{-1} \circ\left(-z_{1}\right)$ maps the interval $[\bar{\theta},+\infty)$ into $[\bar{\theta}, \overline{\bar{\theta}})$. The outcome of this inquiry is then that finding a periodic (monetary) competitive equilibrium with perfect foresight (satisfying (2.g)) with period $k$ is equivalent to finding a periodic solution of (2.10) with period $k$ such that $\theta_{t}>\bar{\theta}$ for all $t$. Indeed, under assumptions $(1 . a),(1 . c)$ and $(2 . g)$, if $\left(p_{1}^{*}, \ldots, p_{k}^{*}\right)$ is a periodic competitive equilibrium with period $k$, and if one define $\theta_{i}^{*}=p_{i}^{*} / p_{i+1}^{*}$ for $i=1, \ldots, k$ (with $p_{k+1}^{*}=p_{1}^{*}$ ), then $\theta_{i}^{*}>\bar{\theta}$ for all $i$ and $\left(\theta_{k}^{*}, \ldots, \theta_{2}^{*}, \theta_{1}^{*}\right)$ is a periodic orbit of $\varphi$ with period $k$. Conversely if $\left(\theta_{k}^{*}, \ldots, \theta_{1}^{*}\right)$ is a periodic orbit of $\varphi$ with period $k$, and if $\theta_{i}^{*}>\bar{\theta}$ for all $i=1, \ldots, k$, then the prices $\left(p_{1}^{*}, \ldots, p_{k}^{*}\right)$ defined by using (2.7) i.e. $p_{i}^{*}=M / z_{2}\left(\theta_{j-1}^{*}\right)$ for $i=1, \ldots, k$ (with $\theta_{0}^{*}=\theta_{k}^{*}$ ) determine a periodic competitive equilibrium with period $k^{l}$. It goes without saying that

This shows incidentally that the prices $\left(p_{1}^{*}, \ldots, p_{k}^{*}\right)$ that compose the orbit of a periodic competitive equilibrium with perfect foresight, with primitive period $k$, must all differ. 
the equivalence we just stated concerns only periodic competitive equilibria and periodic orbits of $\varphi$. By contrast, the "backward rational expectations" dynamics that are implied by (2.10) do not represent any "true" dynamics. The only dynamics that actually describe the evolution of this economy over time is represented by the equation (2.4) or equivalently by the map $\widetilde{N}$.

It may be worthwhile to end up this section with a simple graphical illustration of the backward dynamics associated to (2.10) and the map $\varphi$, by using a trader's offer curve. This is done below in Figure 3, which is drawn under the assumption $\bar{\theta}<1$. Starting with $\theta_{t}>\bar{\theta}$, we first draw the intertemporal budget line of equation $\theta_{t} a_{1}+a_{2}=\theta_{t} \ell_{1}^{*}+\ell_{2}^{*}$. It intersects the trader's offer curve at the point of coordinates $\left(\ell_{1}^{*}+z_{1}\left(\theta_{t}\right), \ell_{2}^{*}+z_{2}\left(\theta_{t}\right)\right)$. Finding the value of $\theta_{t-1}$ that satisfies $z_{1}\left(\theta_{t}\right)+z_{2}\left(\theta_{t-1}\right)=0$ is then achieved by following the arrows on the Figure, by going first vertically to the $45^{\circ}$ line $A B$ that passes through the endowment point $\left(\ell_{1}^{*}, \ell_{2}^{*}\right)$, and then horizontally back to the offer curve. This procedure yields the point of coordinates $\left(\ell_{1}^{*}+z_{1}\left(\theta_{t-1}\right), \ell_{2}^{*}+z_{2}\left(\theta_{t-1}\right)\right)$ and thus the corresponding value of $\theta_{t-1}=\varphi\left(\theta_{t}\right)$ by drawing the corresponding intertemporal budget line. The Figure shows that $\varphi$ has always a fixed point $\theta=\bar{\theta}$, which corresponds to a nonmonetary stationary state, and that $\theta=1$ is the unique monetary stationary state whenever $\bar{\theta}<1$. The Figure gives moreover an example of a cycle of period 2.

\section{Figure 3}


$-32-$

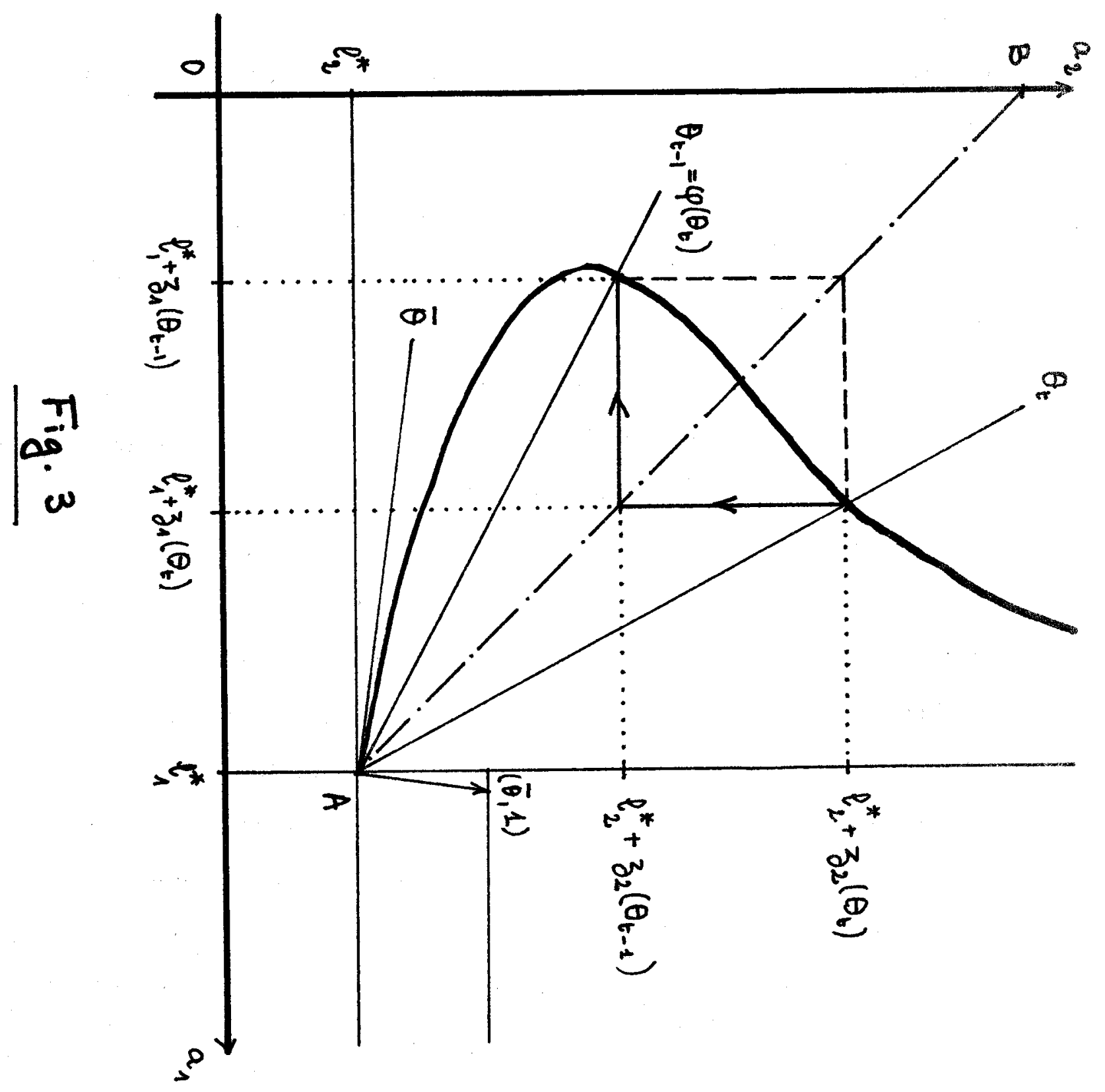


Remarks. 1. Under assumptions (1.a) and (1.c), the backward perfect foresight dynamics that is implied by $(2.6)$ is well defined, as we have seen, and is described by the map $\varphi$. On the other hand, it is easily seen that a "forward perfect foresight" dynamics that would yield $\theta_{t}$ as a function of $\theta_{t-1}$ is not well defined by that equation. Figure 3 gives an example of multiple solutions since trying to go forward from $\theta_{t-1}$ would give the choice among two possible points on the offer curve. Indeed, if one starts from the point of the offer curve that corresponds to $\theta_{t-1}$, the forward dynamics associated to the equation $z_{1}\left(\theta_{t}\right)+z_{2}\left(\theta_{t-1}\right)$ requires that one should go first horizontally to the $45^{\circ}$ line $A B$ and then vertically back to the offer curve. In the case of Fig. 3, this procedure yields two points. If one goes back to Fig. 2.a, one sees easily that the forward dynamics implied by (2.6) is eventually undefined in that case, if one starts with a value of $\theta$ that is greater than 1 . The reason is that at some stage one should have $z_{2}\left(\theta_{t-1}\right)>l_{1}^{*}$, and thus there could not exist a $\theta_{t}$ such that $z_{1}\left(\theta_{t}\right)+z_{2}\left(\theta_{t-1}\right)=0$.

2. The assumption that traders use the same expectation function $\psi$ over time may be interpreted as follows. Consumers have a given "model" of the workings of the economy and use a given statistical technique (Bayesian methods or maximum likelihood procedures) to estimate the parameters of the model and to forecast future prices. This way of proceeding yields the map $\psi$. One might envision more sophisticated learning processes in which traders change over time their models of the economy (their expectation functions) according to some prespecified rule that would for instance take into account previous forecasting errors. Note that this supposes an exchange of information between generations about which "model" they use. Moreover, the description of the state of the economy 
at each date involves then not only the current price $p_{t}$ but also the expectation function $\psi_{t}$ that consumers are employing currently. Such learning procedures have been considered by Fuchs (1976, 1977a, 1977b, $1979 a, 1979 b)$. 


\section{STABILITY AND LEARNING.}

The example given in Figure 3 suggests that several periodic competitive equilibria involving different periods will typically coexist. In the particular case of Fig. 3, a cycle of period 2 coexists with the unique stationary (monetary) equilibrium $\theta=1$. We shall say more on this question in the next section, when analyzing the existence and multiplicity of periodic equilibria. But this preliminary remark shows that the issue of stability is an important one. The purpose of this section is to study more precisely this issue. Specifically, it will be shown that while stability of a periodic equilibrium must take into account that traders learn over time - i.e. it must be defined by using the temporary Walrasian equilibrium function $W$ or equivalently the map $\tilde{W}$ - such stability may still be studied fruitfully by looking at the simpler but fictitious backward perfect foresight dynamics implied by (2.6) or its associated map $\varphi$.

We sha11 assume throughout this section assumptions $(1 . a),(1 . c)$, as well as $(2.6)$, so that the functions $\tilde{W}$ (or $W$ ) and $\varphi$ are continuously differentiable. Let us first define stability. Consider accordingly a periodic competitive equilibrium $\left(p_{1}^{*}, \ldots, p_{k}^{*}\right)$ with period $k$, and the corresponding cycle $\left(q_{1}^{*}, \ldots q_{k}^{*}\right)$ of the map $\tilde{W}$ (see Lemma 2.2.). Since the dynamics implied by the functions $W$ and $\tilde{W}$ are equivalent, we may say that the periodic equilibrium $\left(p_{1}^{*}, \ldots, p_{k}^{*}\right)$ is locally stable if and only if the cycle $\left(q_{1}^{*}, \ldots, q_{k}^{*}\right)$ itself is locally stable. Or if and only if there exists a neighbourhood $Q$ of $q_{1}^{*}$ such that for all $q$ in $Q$, the orbit of $q$ by the map $\tilde{W}^{k}$ stays in $Q$ and converges to $q_{1}^{*}$, i.e. $\tilde{W}^{k t}(q) \in Q$ for $a 11 t \geqq 1$ and $\lim _{t \rightarrow+\infty} \tilde{W}^{k t}(q)=q_{1}^{*}$ (by continuity of $\tilde{W}$, this ensures 
that $\tilde{W}^{k t}\left(\tilde{W}^{i-1}(q)\right)$ converges to $q_{j}^{*}$ as well for $\left.i=2, \ldots, k\right)$. Since under (2. 6), $W$ and $\tilde{W}$ are continuously differentiable, the foregoing stability condition is equivalent to stating that the eigenvalues of the Jacobian matrix of $\tilde{W}^{k}$ at $q_{1}^{*}$, i.e. $\tilde{D W}^{k}\left(q_{1}^{*}\right)$, have all a modulus less than unity.

In order to make sense, the definition should not depend upon whether we start near $q_{1}^{*}$ or near any other point $q_{j}^{*}$ of the periodic orbit $\left(q_{1}^{*}, \ldots, q_{k}^{*}\right)$ of $\tilde{W}$, since $\left(q_{j}^{*}, \ldots, q_{k}^{*}, q_{1}^{*}, \ldots, q_{j-1}^{*}\right)$ describes the same cycle. From $\tilde{W}^{k}(q)=\tilde{W}^{k-1}(\tilde{W}(q))$, we get by employing the chain rule of differentiation,

$$
D \tilde{W}^{k}\left(q_{1}^{*}\right)=D \tilde{W}^{k-1}\left(q_{2}^{*}\right) D \tilde{W}\left(q_{1}^{*}\right)=\ldots=D \tilde{W}\left(q_{k}^{*}\right) \ldots D \tilde{D W}\left(q_{1}^{*}\right)
$$

Thus $\operatorname{DW}^{k}\left(q_{j}^{*}\right)$ is obtained as the product of the Jacobian matrices of $\tilde{W}$, $D \tilde{W}\left(q_{j}^{*}\right)$, at the different points of the cycle. Applying the same procedure to $D \tilde{W}^{k}\left(q_{j}^{*}\right)$, we obtain

$$
D_{W^{k}}\left(q_{i}^{*}\right)=D \tilde{W}\left(q_{i-1}^{*}\right) \ldots D \tilde{W}\left(q_{1}^{*}\right) D \tilde{W}\left(q_{k}^{*}\right) \ldots D \tilde{W}\left(q_{j}^{*}\right)
$$

Now for any two square matrices $A$ and $B$, the eigenvalues of the products $A B$ and $B A$ are the same (see e.g. Wilkinson (1965) p. 54), so that the eigenvalues of the Jacobian matrices $D \tilde{W}^{k}\left(q_{1}^{*}\right), \ldots, D \tilde{W}^{k}\left(q_{k}^{*}\right)$, are all the same, which establishes the equivalence we were looking for. We have thus the following result, which will be taken as a definition.

$$
\text { Under assumptions }(1, a),(1 . c) \text { and }(2.6) \text {, the periodic equilibrium }
$$
$\left(p_{1}^{*}, \ldots, p_{k}^{*}\right)$ is leocally) stable if and only if the eigenvalues of the Jacobian matrix

$$
\operatorname{DW}^{k}\left(q_{1}^{*}\right)=\operatorname{DW}\left(q_{k}^{*}\right) \ldots D \tilde{D}\left(q_{1}^{*}\right)
$$


Computing the eigenvalues of a $(T \times T)$ matrix - or checking that they have a modulus less than 1 - is not a very pleasant task when $T$ is large. But we have shown in the preceding section that under assumption (2.9), one may associate to the periodic equilibrium $\left(\mathrm{p}_{1}^{*}, \ldots, \mathrm{p}_{\mathrm{k}}^{*}\right)$ the cycle $\left(\theta_{\mathrm{k}}^{*}, \ldots, \theta_{1}^{*}\right)$ of the map $\varphi$ defined in $(2.10)$, through the relations $\theta_{i}^{*}=p_{i}^{*} / p_{i+1}^{*}\left(\right.$ with $\left.p_{k+1}^{*}=p_{1}^{*}\right)$ - and conversely. One may hope to exploit this equivalence by looking at the stability of the cycle $\left(\theta_{k}^{*}, \ldots, \theta_{1}^{*}\right)$ under the dynamics implied by $\varphi$. Indeed, stability in that case is much simpler. If we transpose the argument given above concerning the local stability of the periodic orbit $\left(q_{1}^{*}, \ldots, q_{k}^{*}\right)$ of the map $\tilde{W}$, we see that $\left(\theta_{k}^{*}, \ldots, \theta_{1}^{*}\right)$ is (lucally) $\varphi$-stable if and only if the absolute value of the derivative of $\varphi^{k}$ at $\theta_{k}^{*}$, i.e. $\left|D \varphi^{k}\left(\theta_{k}^{*}\right)\right|$, is less than 1 . Again, this definition does not depend upon the point chosen on the orbit since by the chain rule of differentiation,

$$
\begin{aligned}
D \varphi^{k}\left(\theta_{k}^{*}\right) & =D \varphi^{k-1}\left(\theta_{k-1}^{*}\right) D \varphi\left(\theta_{k}^{*}\right) \\
& =D \varphi\left(\theta_{1}^{*}\right) \ldots D \varphi\left(\theta_{k}^{*}\right) \\
& =D \varphi^{k}\left(\theta_{i}^{*}\right)
\end{aligned}
$$

The hope to ascertain some relation between the stability of a periodic equilibrium $\left(p_{1}^{*}, \ldots, p_{k}^{*}\right)$ and $\varphi$-stability may seem somewhat foolish at first sight. After a11, the map $\varphi$ describes a fictitious and very abstract backward dynamics with perfect foresight, while the actual dynamics given by $W$ or $\tilde{W}$ go "forward". The enterprise is not as vain as one might expect, however. Under assumption(2.g), anticipations are correct along a periodic orbit, and thus by continuity, nearly correct in its neighbourhood. On the other hand, the essence of learning is precisely to forecast future prices by looking backward at past prices. 
Indeed let us consider

(3.h) For any periodic sequence $\left(p_{t}, p_{t-1}, \ldots, p_{t-T}\right)$ that has period $k$, the expectation function satisfies $\psi_{0}^{\prime}=0$ and $\psi_{j}^{\prime} \geqq 0$ for all $j=1, \ldots, T$.

Then we have the following ${ }^{1}$

PROPOSITION 3.1. Assume $(1 . a),(1 . c)$ and $(2.6),(2 . g)$.

Consider a periodic competitive equilibrium $\left(p_{1}^{*}, \ldots, p_{k}^{*}\right)$ and the associated cycle $\left(\theta_{k}^{\star}, \ldots, \theta_{1}^{*}\right)$ of $\varphi$. Under assumption $(3 . h), \varphi$-stability of $\left(\theta_{k}^{*}, \ldots, \theta_{1}^{*}\right)$ implies stability of $\left(p_{1}^{*}, \ldots, p_{k}^{*}\right)$ in the sense of $(3.1)$.

Assumption $(3 . h)$ says that a trader facing a periodic sequence of current and past prices thinks that a small variation of the current price has only a secondary importance, and that a small increase of past prices does not lead to a downward revision of the expected price. It is instructive to see what are the implications of $(3 . h)$ in the light of assumption (2.g). The latter assumption says that for any periodic sequence $\left(p_{t}, \ldots, p_{t-T}\right)$ with period $k$, one has $\psi\left(p_{t}, \ldots, p_{t-T}\right)=p_{t-k+1}$. Note that this implies that the expected price $\psi$ is then unchanged, for each integer $0 \leqq j \leqq k-2$, whenever the (constant) sequence $\left(p_{t-j}, p_{t-j-k}, \ldots\right.$, $\left.p_{t-j-n k}, \ldots\right)$ is multiplied by an arbitrary real number $\lambda>0$, and that $\psi$ changes proportionately to $\lambda$ when $j=k-1$. When $\psi$ is differentiable, one gets then by differentiation with respect to $\lambda$ in each case

$$
\psi_{j}^{\prime}+\psi_{j+k}^{\prime}+\ldots+\psi_{j+n k}^{\prime}+\ldots=0
$$

The proof of this claim, which is mostly computational, is given in the Appendix in order to ease the exposition. 
for $0 \leqq j \leqq k-2$ (here $n$ runs from 0 to the largest integer that is compatible with $j+n k \leqq T$ ) and

$$
\psi_{k-1}^{\prime}+\psi_{2 k-1}^{\prime}+\ldots+\psi_{n k-1}^{\prime}+\ldots=1
$$

in which $n$ runs this time from 1 to the largest integer compatible with $n k-1 \leqq T$ (we recall that we consider $T$ as finite but "large"). The arguments of these partial derivatives are of course the particular periodic sequence $\left(p_{t}, \ldots, p_{t-T}\right)$ under consideration. In view of these relations, assumption (3.h) implies then that

(3.4) $\psi_{0}^{\prime}=0$. All other partial derivatives $\psi_{j}^{\prime}$ are zero, except those of the form $\psi_{n k-1}^{\prime}, n \geqq 1$, which are nonnegative and satisfy (3.3)

What this condition means is that a trader facing a periodic sequence of prices $\left(\bar{p}_{t}, \ldots, \bar{p}_{t-T}\right)$ with period $k$ behaves locally (in a neighourhood of the sequence) as if movements of the current prices, and of the past prices other than those of the form $p_{t-n k+1}$, were only of secondary (negligible) importance. His forecast is then - again locally - some sort of "average" of the relevant past prices $p_{t-k+1}$, $p_{t-2 k+1}$, and so on. Indeed, for any (even aperiodic) sequence $\left(p_{t}, \ldots, p_{t-T}\right)$ in a neighbourhood of the original periodic sequence $\left(\bar{p}_{t}, \ldots, \bar{p}_{t-T}\right)$, the expected price is approximately given by

$$
\psi\left(p_{t}, \ldots, p_{t-T}\right) \simeq \psi\left(\bar{p}_{t}, \ldots, \bar{p}_{t-T}\right)+\sum_{j=0}^{T}\left(p_{t-j}-\bar{p}_{t-j}\right) \psi_{j}^{\prime}\left(\bar{p}_{t}, \ldots, \bar{p}_{t-T}\right)
$$

which yields in view of (3.4) 


$$
\psi\left(p_{t}, \ldots, p_{t-T}\right) \simeq \sum_{n \geqq 1} p_{t-n k+1} \psi_{n k-1}^{\prime}\left(\bar{p}_{t}, \ldots, \bar{p}_{t-T}\right)
$$

(the first term of this sum being 0 if $k=1$ ). Such a behaviour does not sound implausible ${ }^{1}$.

Proposition 3.1 will lead to a great simplification of our analysis in the sequel. For it tells us that if we succeed in finding a cycle $\left(\theta_{k}^{*}, \ldots, \theta_{1}^{*}\right)$ of the map $\varphi$ that is stable, the corresponding periodic equilibrium $\left(p_{1}^{*}, \ldots, p_{k}^{*}\right)$ is automaticably stable itself, provided that anticipations verify $(3 . h)$. It should be emphasized however, that the result goes one way only. It is possible in particular that a cycle $\left(\theta_{k}^{*}, \ldots, \theta_{1}^{*}\right)$ is unstable in the dynamics associated to $\varphi$, whereas the corresponding periodic equilibrium $\left(p_{1}^{*}, \ldots, p_{k}^{*}\right)$ is stable.

As a matter of fact, Proposition 3.1 is still valid if $(3 . h)$ is replaced by the weaker condition that $(3.4)$ is satisfied at all sequences $\left(p_{t}, \ldots, p_{t-T}\right)$ originating from the infinite periodic sequence of equilibrium prices $\left(p_{t}\right)$ that is associated to the given periodic equilibrium $\left(p_{1}^{*}, \ldots, p_{k}^{*}\right)$ (the proof of the Proposition uses only that condition). It should be noted on the other hand, that the concept of stability is "structurally stable", i.e. stability is preserved if the expectation function is slightly and "smoothly" perturbated ("smoothly" meaning that $\psi$ and its partial derivatives undergo a slight change). In particular, the validity of Proposition 3.1 is preserved if $\psi$ satisfies only approximatively $(3 . h)$ (is close enough to a function satisfying $(3 . h)$ in the $c^{1}$ topology) provided that it still satisfies $(2 . \mathrm{g})$. We do not elaborate on this point. 
Another outcome of the foregoing analys is is that one should generally be very cautious when interpreting the stability results that are obtained in a model employing the convenient but abstract assumption that traders have perfect foresight even out of "steady states" - as has been customary recently under the impulse of the so-called "New Macroeconomics" school. For if the traders' learning processes are taken explicitly into account when modelling the dynamic evolution of an economy - as they should since learning is after all the primitive concept that enables model builders to justify at least implicitly the mere assumption of perfect foresight - then stability results may well be reversed. The point is most forcedfully illustrated by the case represented in Fig. 2,a. As we have already seen (see the Remark at the end of Section 2), the "forward" perfect foresight dynamics described by the equation $z_{1}\left(\theta_{t}\right)+z_{2}\left(\theta_{t-1}\right)=0$ is then ultimately undefined if one starts with $\theta>1$. But at least such dynamics is well defined in a neighbourhood of the unique stationary monetary equilibrium $\theta=1$

- it can then be approximated by the 1 inearized system $\left(\theta_{t}-1\right)=\gamma\left(\theta_{t-1}-1\right)$ in which $\gamma=-\left(z_{2}^{\prime}(1) / z_{1}^{\prime}(1)\right)$. By differentiating (1.4) at $\theta=1$, we get $z_{2}^{\prime}(1)+z_{1}(1)+z_{2}^{\prime}(1)=0$, and thus $\gamma>1$. The stationary equilibrium $\theta=1$ is unstable in the forward perfect foresight dynamics (equivalently, it is $\varphi$-stable). Suppose now that traders use in fact a learning process to form their forecasts and that the associated expectation function $\psi$ satisfies for every stationary sequence of prices $\left(p_{t}=p\right.$ for all $\left.t\right) \psi(p, p, \ldots, p)=p$, that it does not depend on the current price and that it is a nondecreasing function of past prices. Then $\psi$ satisfies $(2 . g)$ and $(3 . h)$, and Proposition 3.1 tells us that the stationary equilibrium, being $\varphi$-stable, is indeed stable when traders employ the above learning process, which appears quite reasonable. 
Remark. Stability of a stationary monetary equilibrium has been studied in the more general context of temporary equilibrium theory by Fuchs and Laroque (1976) - see also Tillman (1983). The issue of stability of a stationary equilibrium when traders employ more sophisticated learning procedures - in particular when they may revise their expectation functions over time in view of previous forecasting errors - has been investigated by Fuchs (1976, 1977a, 1977b, 1979a, 1979b). Stability of a periodic competitive equilibrium when consumers revise their expectations functions over time, and its relation to $\varphi$-stability is an open issue in that case. Note that the problem becomes much more complicated, since the state of the economy at a given date must then be described by a price and an expectation function. The paper by Fuchs and Laroque (1976) contains also a few partial results concerning local dynamics in a neighbourhood of a given cycle.

The question of the stability of perfect foresight or rational expectations equilibria when traders employ a given learning procedure has also been addressed to in a macroeconomic stochastic model by M. Bray (1982), P. Champsaur (1983), B. Friedman (1979). 
4. EXISTENCE, STABILITY AND BIFURCATION OF PERIODIC EQUILIBRIA.

The results that were obtained in the preceding two Sections imply first that finding a periodic monetary equilibrium with perfect foresight is equivalent to finding a periodic orbit of the map $\theta_{t-1}=\varphi\left(\theta_{t}\right)$ with $\theta_{t}>\bar{\theta}$ for all $t$, and second that $\varphi$-stability implies stability of the true dynamics with learning described by $W$ or $\widetilde{W}$, if expectations satisfy assumption (3.h). We take advantage of these facts and study in the present Section the conditions that lead to the occurrence of cycles of the map $\varphi$, their multiplicity and stability, without any further explicit reference to the underlying dynamical process with learning. The tools that we shall use are borrowed from recent mathematical theories that have been constructed by using partly the notion of the "Hopf's bifurcation" of a dynamical system in order to explain the emergence of cycles and the transition to turbulent ("chaotic" or aperiodic) behaviour in physical, biological or ecological systems ${ }^{1}$.

The general idea is that cycles with a period $k \geqq 2$ will appear in the present model whenever there is an important conflict between the intertemporal substitution effect and the wealth effect that results from a variation of the real interest rate, so that a trader's offer curve

\footnotetext{
One important mathematical reference in this field is collet and Eckmann (1980). For an excellent review of various applications of the theory, see May (1976). Part of this theory has been already applied in economics or game theory in particular by Benhabib and Day (1981, 1982), Dana and Malgrange (1981), Day (1982, 1983), Jensen and Urban (1982), Rand (1978). The results of this theory that seemed (to me) relevant and useable by economic theorists are reviewed in Grandmont (1983b).
} 
displays a significant "hump" as in Fig. 3. For this to be the case, it is necessary that an old trader's relative degree of risk aversion $R_{2}\left(a_{2}\right)$ is greater than 1 for some $a_{2}>0$. The phenomenon will be seen to appear in particular - in the case of a constant relative risk aversion - when old consumers are sufficiently more risk averse than young traders.

An interesting fact occurs, however, as cycles with different periods will typically coexist. An instance of the phenomenon was already given Fig. 3, in which a cycle with period 2 coexisted with the unique monetary steady state. Much more can be said in fact. If the set of positive integers is ordered in a specific way - which we may call the "Sarkovskij's ordering" from the name of the mathematician who discovered it - and if the map $\varphi$ has a cycle of period $k$, then we are sure that it has also a cycle with a period $k^{\prime}$ in which $k^{\prime}$ is any integer that is ranked before $k$ in the ordering. In particular, if there is a cycle of period $2^{k}$, then there is also periodic orbit with period $2^{n}$ in which $n=0,1, \ldots, k-1$. Moreover, if there is a cycle of period 3 , then cycles of period $n$, in which $n$ is an arbitrary positive integer, will also exist.

In the face of this bewildering multiplicity of periodic equilibria, the stability issue is essential. There is indeed a condition on the map $\varphi$, namely that it has a negative "Schwarzian derivative", that ensures that there exists at most one stable periodic orbit. This condition will be related to certain properties of the traders' utility functions. It will be seen in particular that the condition obtains, in the case of a constant relative degree of risk aversion, when young 
consumers are only moderately risk averse (have a relative risk ayersion that is less than or equal to 1 ) while old traders haye a relative risk aversion greater than or equal to 2. Needless to say, this result, as instructive as it is, does not imply in general however that the true dynamics with learning described by the map $W$ or $\tilde{W}$ possess a unique stable cycle.

The last part of the Section will be devoted to the analysis of the "bifurcations" of the dynamical system, and more precisely of the emergence of $\varphi$-stable cycles. It will be seen essentially that if one considers a one parameter family of economies, then stable periodic orbits do appear once again in conformity to the Sarkovskij's ordering of the set of integers. We shall give a particular example of such a bifurcation scheme in the case of a constant relative risk aversion, in which the young consumers' risk aversion is held fixed, while the old traders' risk aversion is increased progressively. The result of this computer experiment will be that cycles do emerge for values of the relative degrees of risk aversion that do not appear implausible.

Characteristics of the map $\varphi$ and equivalent dynamics.

We begin the analysis by reviewing a few basic properties of the map $\varphi$ and define dynamical systems that are equivalent to the difference equation $\theta_{t-1}=\varphi\left(\theta_{t}\right)$. These equivalent dynamical systems are in fact obtained by making a change of variable $\tilde{\theta}=h(\theta)$.

We assume throughout this Section $(1 . a),(1 . c)$. Then the function 


$$
\varphi=z_{2}^{-1}\left(-z_{1}(\theta)\right)=z_{2}^{-1}\left(z_{2}(\theta) / \theta\right)
$$

maps the interval $[\bar{\theta},+\infty)$ into $[\bar{\theta}, \overline{\bar{\theta}})$ in which $\overline{\bar{\theta}}=z_{2}^{-1}\left(\ell_{1}^{*}\right)$, and is continuously differentiable. Clearly $\bar{\theta}$ is a fixed point of $\varphi$, that corresponds to a nonmonetary stationary equilibrium. On the other hand, we have $\varphi(\theta)<\theta$ whenever $\theta>1$. Thus if $\bar{\theta} \geqq 1$, there can be no monetary cycle of any period since $\varphi^{j}(\theta)$ decreases monotonically to $\bar{\theta}$ as $j$ tends to infinity. We shall assume accordingly $\bar{\theta}<1$ (assumption (1.d)). We have then

LEMMA 4.1. Assume (1.a), (1.c), (1.d). Then $\varphi$ maps the interval $[\bar{\theta},+\infty)$ into $[\bar{\theta}, \overline{\bar{\theta}})$ in which $\overline{\bar{\theta}}=z_{2}^{-1}\left(\ell_{1}^{*}\right)$, and is continuously differentiable. Moreover

1) $\varphi(\bar{\theta})=\bar{\theta}, \varphi(1)=1, \varphi(\theta)>\theta$ whenever $\bar{\theta}<\theta<1$ and $\varphi(\theta)<\theta$ whenever $\theta>1$

2) One has $\varphi^{\prime}(\bar{\theta})=1 / \bar{\theta}>1$ and $\varphi^{\prime}(1)<1$

3) Let $\alpha_{2}=\operatorname{Sup} R_{2}\left(a_{2}\right)$. Then if $\alpha_{2} \leqq 1$, one has $\varphi^{\prime}(\theta)>0$ for all $\theta \geqq \bar{\theta}$.

4) If $\alpha_{2}>1$ and if $R_{2}\left(a_{2}\right)$ is nondecreasing lassumption (1.e)), then the map $\varphi$ is unimodal with a unique nondegenerate critical point, i.e. there exists $\theta^{*}>\bar{\theta}$ such that $\varphi^{\prime}(\theta)>0$ when $\bar{\theta} \leqq \theta<\theta^{*}, \varphi^{\prime}\left(\theta^{*}\right)=0$ and $\varphi^{\prime}(\theta)<0$ when $\theta>\theta^{*}$. In that case, the conditions $\varphi^{\prime}(1)<0$ or $\theta^{*}<1$ or $\theta^{*}<\varphi\left(\theta^{*}\right)$ are equivalent. If anyone of them is satisfied, one has $\theta^{*}<1<\varphi\left(\theta^{*}\right)$ and $\varphi^{2}\left(\theta^{*}\right)<1<\varphi\left(\theta^{*}\right)$. 
The proof of the lemma is straightforward, The first part follows trivially from (4.1) and the fact that $z_{2}$ is an increasing function. As for the last two points, it suffices to remark that

$$
\varphi^{\prime}(\theta)=-z_{1}^{\prime}(\theta) / z_{2}^{\prime}(\varphi(\theta))
$$

which can be rewritten by differentiation of the identity $\theta z_{1}(\theta)+z_{2}(\theta)=0$

$$
\varphi^{\prime}(\theta)=\left[z_{2}^{\prime}(\theta)-\left(z_{2}(\theta) / \theta\right)\right] /\left[\theta z_{2}^{\prime}(\varphi(\theta))\right]
$$

Thus (4.3) yields $\varphi^{\prime}(\theta)=1 / \bar{\theta}>1$ since $z_{2}(\bar{\theta})=0$ and $\varphi^{\prime}(1)<1$. Finally, the point 3) and the first part of 4) are obvious consequences of (4.2) and of lemma 1.3. The last part of 4) is true for any unimodal map $\varphi$ that has $\theta=1$ as a fixed point.

The map $\varphi$ seems to be the most natural to consider from an economic viewpoint since it originates from the perfect foresight equilibrium equation for the good market (2.6), which determines equilibrium relative prices and thus real interest rates independently of the money sector. Yet there is nothing intrinsic from a mathematical point of view that is attached to this particular function, and its will be sometimes convenient in the sequel to modify the foregoing dynamical equation $\theta_{t-1}=\varphi\left(\theta_{t}\right)$ by making a change of variable of the form $\tilde{\theta}=h(\theta)$ in which the function $h$ maps $[\bar{\theta},+\infty)$ onto some interval $[a, b)$, is continuously differentiable, and $h^{\prime}(\theta)>0$ for all $\theta \geqq \bar{\theta}$. The equation $\theta_{t-1}=\varphi\left(\theta_{t}\right)$ becomes then with the new variable 


$$
\tilde{\theta}_{t-1}=h\left[\varphi\left(h^{-1}\left(\tilde{\theta}_{t}\right)\right)\right] \text { or } \tilde{\theta}_{t-1}=\tilde{\varphi}\left(\tilde{\theta}_{t}\right) \text { with } \tilde{\varphi}=h o \varphi h^{-1}
$$

The two maps $\varphi$ and $\tilde{\varphi}$ are then said to be topological conjugates. It is clear that they describe the same dynamics : this follows from the relation between the iterates of the two conjugate maps, that is $\tilde{\varphi}^{k}=h_{0} \varphi^{k} \circ h^{-1}$. In particular, the two maps have the same cycles up to the change of variable formula $\tilde{\theta}=h(\theta)$, and the stability or unstability of a cycle is unaltered by the change of coordinates ${ }^{1}$.

We shall take advantage sometimes of this equivalence by considering a particular change of variable, in which $h(\theta)=z_{2}(\theta)$, and sha11 denote the result $x=z_{2} \circ \varphi_{0} z_{2}^{-1}$. One may remark that the function $x$ has a particularly simple interpretation, for it describes the (backward) perfect foresight dynamics on equilibrium real balances. Indeed, we know from (2.7) that these equilibrium real balances $\mu_{t}$ are associated to perfect foresight equilibrium real interest rates $\theta_{t}$ by $\mu_{t+1}=z_{2}\left(\theta_{t}\right)$. Therefore the equation $\theta_{t-1}=\varphi\left(\theta_{t}\right)$ becomes through this transformation

$$
\mu_{t}=z_{2}\left(\theta_{t-1}\right)=\mu_{t+1} / z_{2}^{-1}\left(\mu_{t+1}\right)=x\left(\mu_{t+1}\right)
$$

More precisely, it is easy to verify by differentiation that

$$
D \tilde{\varphi}^{k}(h(\theta))=h^{\prime}\left(\varphi^{k}(\theta)\right) D \varphi^{k}(\theta) / h^{\prime}(\theta)
$$

Thus the sign of derivatives is unchanged. Moreover, if $\theta$ is a fixed point of $\varphi^{k}, h(\theta)$ is a fixed point of $\tilde{\varphi}^{k}$, and $D_{\varphi}^{\sim k}(h(\theta))=D \varphi{ }^{k}(\theta)$.

Finally, it is readily seen by inspection that $\tilde{\varphi}^{k}(h(\theta))>h(\theta)$ if and only if $\varphi^{k}(\theta)>\theta$. 
The curves describing the maps $\varphi$ and $\chi$ are represented below in Figures 4.a and 4.b under assumptions $(1 . a),(1 . c),(1 . d)$, (1.e), and when $\alpha_{2}=\operatorname{Sup} R_{2}\left(a_{2}\right)>1$. Of course, since the two curves are deduced from each other through the change of variable $\mu=z_{2}(\theta)$, they must display the same qualitative properties. In particular, if $\alpha_{2}=\operatorname{Sup} R_{2}\left(a_{2}\right) \leqq 1, x$ is increasing everywhere, while $x$ has a unique critical point $\mu^{*}=z_{2}\left(\theta^{*}\right)$ when $\alpha_{2}>1$ under assumption $(1 . e)^{1}$.

Fig. 4.2

Fig. 4.b

It is useful to note that the map $\varphi$ (or $x$ ) may be defined implicitly by an equation involving marginal utilities. If we look back at the identity (1.11) and apply it for $\theta=\theta_{t}$, we see that the backward difference equation $z_{1}\left(\theta_{t}\right)+z_{2}\left(\theta_{t-1}\right)=0$ is equivalent to

$$
z_{2}\left(\theta_{t-1}\right) v_{1}^{\prime}\left(l_{1}^{*}-z_{2}\left(\theta_{t-1}\right)\right)=z_{2}\left(\theta_{t}\right) v_{2}^{\prime}\left(l_{2}^{*}+z_{2}\left(\theta_{t}\right)\right)
$$

which gives the function $\varphi$ in implicit form. By making the change of variable $\mu_{t+1}=z_{2}\left(\theta_{t}\right),(4.5)$ becomes

The transposition of lemma 4.1 into the corresponding properties of $x$ is straightforward and is left to the reader. One may remark that the curve representing the map $x$ is obtained simply from the trader's offer curve by making a rotation of angle $\frac{\pi}{2}$ in the plane $\left(a_{1}, a_{2}\right)$ around the endowment point $\left(\ell_{1}^{*}, \ell_{2}^{*}\right)$ - see Fig. 3 . 

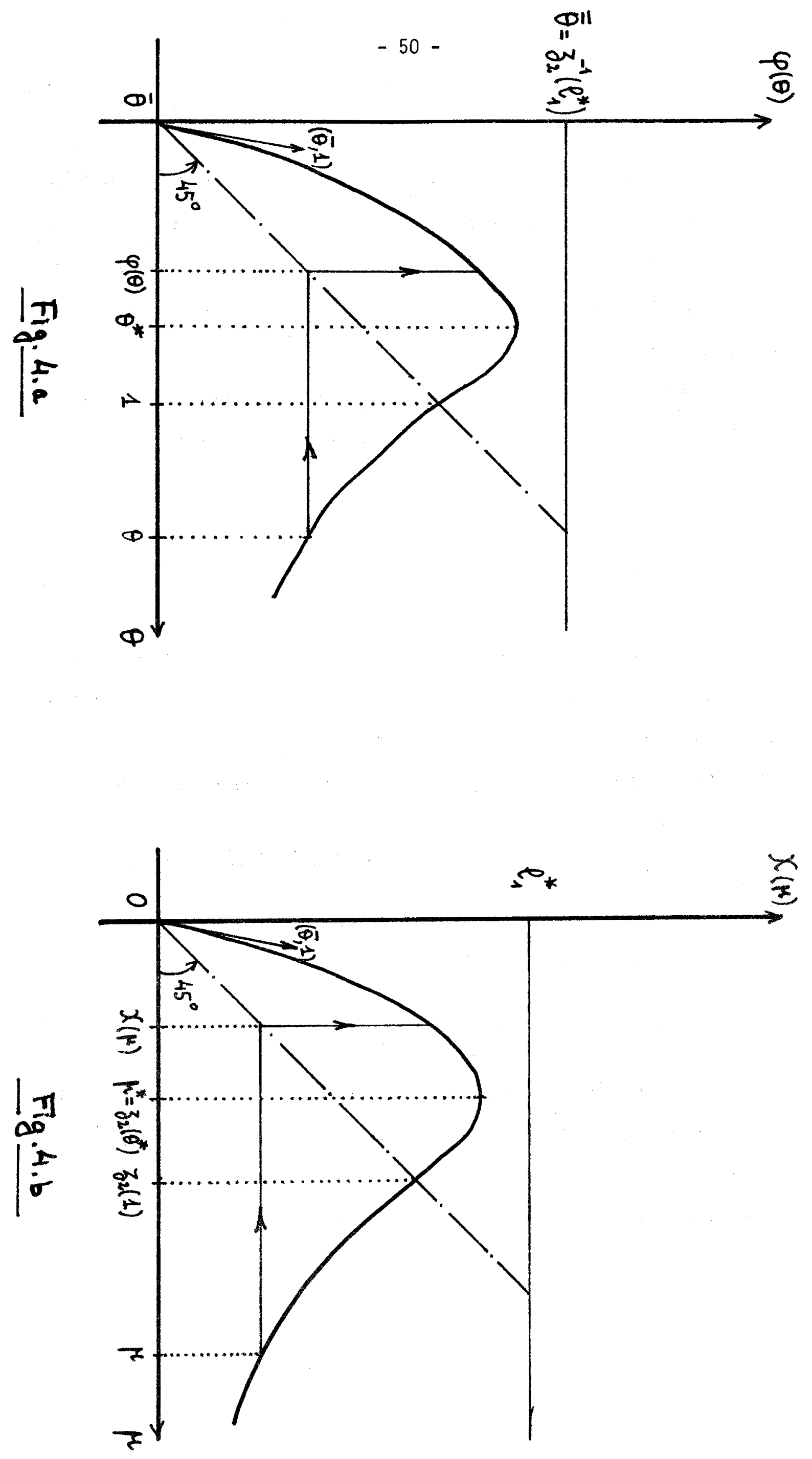


$$
{ }^{\mu} V_{1}\left(l_{1}^{*}-\mu_{t}\right)={ }^{\mu}{ }_{t+1} V_{2}^{\prime}\left(l_{2}^{*}+\mu_{t+1}\right)
$$

which defines implicitly the map $x$. As a matter of fact, if we define the functions $v_{1}$ and $v_{2}$ by

$$
\begin{aligned}
& v_{1}(\mu)=\mu V_{1}\left(\ell_{1}^{*}-\mu\right) \text { for } \mu \text { in }\left[0, \ell_{1}^{*}\right) \text { and } \\
& v_{2}(\mu)=\mu v_{2}\left(\ell_{2}^{*}+\mu\right) \text { for all } \mu \geqq 0
\end{aligned}
$$

we see that $v_{1}$ is a differentiably increasing function that maps the interval $\left[0, \ell_{1}^{*}\right)$ onto $[0,+\infty)$, while $v_{2}$ maps $[0,+\infty)$ into itself. Then one has $x(\mu)=\left(v_{1}^{-1} \circ v_{2}\right)(\mu)$ for $211 \mu \geqq 0$. This way of defining the function $x$ has the advantage of involving directly the traders' characteristics, i.e. their utility functions, instead of their demand functions $z_{1}$ and $z_{2}$ like in (4.4). This feature will be quite useful in the sequel.

The reader might benefit from relating the properties of the map $x$ to the corresponding properties of the functions $v_{1}$ and $v_{2}$. In particular, if $\alpha_{2}=\operatorname{Sup} R_{2}\left(a_{2}\right) \leqq 1, v_{2}$ is increasing everywhere, while $v_{2}$ has a unique maximum at $\mu=\mu^{*}=z_{2}\left(\theta^{*}\right)$ under assumption (1.e) when $\alpha_{2}>1$. It is moreover easy to verify that

$$
V_{1}(\mu)>V_{1}\left(l_{1}^{*}\right)_{\mu} \text { and } V_{2}(\mu)<V_{2}^{\prime}\left(l_{2}^{*}\right)_{\mu} \text { for a } 11 \mu>0
$$

This statement, which says in effect that the curve representing $v_{1}$ (or $v_{2}$ ) must lie above (or below) its tangent at the origin $\mu=0$, follows directly from $(4.7)$ and the strict concavity of the utility functions $V_{\tau}$. 
Existence and multiplicity of periodic equilibria.

We look now at the existence and multiplicity of periodic equilibria. We shall assume throughout assumptions $(1 . a),(1 . c)$, (1.d), and shall note as before $\alpha_{2}=\operatorname{Sup} R_{2}\left(a_{2}\right)$.

Since $\bar{\theta}<1$, there is a unique stationary monetary equilibrium $\theta=1$. We give first conditions under which there is no other cycle with a period $k \geqq 2$.

LEMMA 4.2. Assume (1.a), (1.c), (1.d). If $\alpha_{2} \leqq 1$, or if assumption (1.e) holds, $\alpha_{2}>1$ and $z_{2}^{\prime}(1) \leqq 0$, then the map $\varphi$ has no cycle with a period $k \geqq 2$. Moreover, the unique stationary monetary equilibrium is globally $\varphi$-stable, i.e. $\lim _{j \rightarrow+\infty} \varphi^{j}(\theta)=1$ for every $\theta>\bar{\theta}$.

The proof of this statement is immediate. Under the assumptions of the lemma, either $\varphi$ is increasing everywhere, or $\varphi$ is unimodal with a unique critical point $\theta^{*} \geqq 1$. Consider now an arbitrary $\theta_{0}$ that differs from $\bar{\theta}$ and from 1 . In the first case, i.e. When $\alpha_{2} \leqq 1$, then if $\theta_{0}<1$ one has $\theta_{0}<\varphi\left(\theta_{0}\right)<\varphi(1)=1$, and one gets the reverse inequalities whenever $\theta_{0}>1$. By applying the same arguments to the iterates $\varphi^{j}\left(\theta_{0}\right)$, one gets that $\varphi^{j}\left(\theta_{0}\right)$ converges monotonically to 1 when $j$ tends to infinity. In the second case, we remark that $\varphi(\theta) \leqq \varphi\left(\theta^{*}\right)$ for all $\theta$, so that we may assume without loss of generality that the initial point $\theta_{0}$ belongs to the interval $\left(\bar{\theta}, \varphi\left(\theta^{*}\right)\right]$. Note next that $\theta^{\star} \geqq 1$ implies $\varphi\left(\theta^{*}\right) \leqq \theta^{*}$. Thus $\varphi^{\prime}(\theta)>0$ for al1 $\theta$ in the interior of the interval $\left(\bar{\theta}, \varphi\left(\theta^{*}\right)\right]$. One may therefore reproduce 
the argument given in the first case to conclude that $\varphi^{j}\left(\theta_{0}\right)$ converges to 1 as $j$ tends to $+\infty$. This global stability result implies of course the inexistence of a cycle displaying a period $k \geqq 2$.

The foregoing result shows in particular that a necessary condition for the occurrence of a cycle of period $k \geqq 2$ (of a "nondegenerate" cycle) is that old traders are sufficiently risk averse $\left(\alpha_{2}>1\right)$ and that (under assumption (1.e)|z $z_{1}^{\prime}(1)>0$, or equivalently that $\varphi^{\prime}(1)<0$. This condition means that the unique critical point of satisfies $\theta^{*}<1$ (equivalently $x\left(\mu^{*}\right)>\mu^{*}$ or $v_{2}\left(\mu^{*}\right)>v_{1}\left(\mu^{*}\right)$ ).

It is straightforward to see that under assumptions (1.a), (1.c), (1.d) a sufficient condition for the existence of a cycle of period 2 is that the stationary equilibrium is $\varphi$-unstable, i.e. $\varphi^{\prime}(1)<-1$, or equivalently $z_{1}^{\prime}(1)>z_{2}^{\prime}(1)^{1}$. A cycle of period 2 is indeed described by $\left(\theta_{1}^{*}, \theta_{2}^{*}\right)$ in which each $\theta_{j}^{*}$ is a fixed point of the iterate $\varphi^{2}$, and $\theta_{2}^{*}=\varphi\left(\theta_{1}^{*}\right) \neq \theta_{1}^{*}$. It is then clear that in order to find a cycle of period 2, it is necessary and sufficient to find a fixed point $\theta>\bar{\theta}$ of $\varphi^{2}$ that differs from 1 - the corresponding orbit being then $(\theta, \varphi(\theta))$. Now the function $\varphi^{2}$ maps the interval $[\bar{\theta},+\infty)$ into $[\bar{\theta}, \overline{\bar{\theta}})$

In terms of the functions $v_{1}$ and $v_{2}$ introduced in (4.7), this condition means $v_{1}^{\prime}\left(z_{2}(1)\right)+v_{2}^{\prime}\left(z_{2}(1)\right)<1$. The reader will note also by using (4.3) that the condition $\varphi^{\prime}(1)<-1$ means that the elasticity of the function $z_{2}$ at $\theta=1$ is less than 0.5 , or equivalently that the elasticity of the demand for real balances $z_{2}(\theta) / \theta$ at $\theta=1$ is less than -0.5 . 
and is continuously differentiable, One has $\varphi^{2}(\bar{\theta})=\bar{\theta}$, the derivative of $\varphi^{2}$ at $\bar{\theta}$ is equal to $(1 / \bar{\theta})^{2}>1$, and of course $\varphi^{2}(1)=1$. A sufficient condition for the existence of a fixed point $\theta>\bar{\theta}$ of $\varphi^{2}$ that differs from 1 is therefore that the derivative of $\varphi^{2}$ at $\theta=1$ is greater than unity, that is

$$
D \varphi^{2}(1)=\left[\varphi^{\prime}(1)\right]^{2}>1
$$

Indeed, $\varphi^{2}(\theta)>\theta$ for $\theta>\bar{\theta}$ provided that $\theta$ is close enough to $\bar{\theta}$. Under (4.9), $\varphi^{2}(\theta)<\theta$ if $\theta$ is less than but close enough to 1 . Thus by continuity, there exists $\theta \neq \bar{\theta}, 1$ such that $\varphi^{2}(\theta)=\theta$. Since $\varphi^{\prime}(1)<1$, the sufficient condition (4.9) means in effect $\varphi^{\prime}(1)<-1$, as announced.

It is instructive to reformulate the sufficient condition $z_{1}^{\prime}(1)>z_{2}^{\prime}(1)$ as follows by using the expressions of the derivatives $z_{1}^{\prime}$ and $z_{2}^{\prime}$ given in lemma 1.2 (or by using directly the equivalent condition $\left.v_{1}^{\prime}\left(z_{2}(1)\right)+v_{2}^{\prime}\left(z_{2}(1)\right)<1\right)$

$$
R_{2}\left(\ell_{2}^{*}+z_{2}(1)\right)>2 \frac{l_{2}^{*}+z_{2}(1)}{z_{2}(1)}+\frac{l_{2}^{*}+z_{2}(1)}{\ell_{1}^{*}+z_{1}(1)} R_{1}\left(l_{1}^{*}+z_{1}(1)\right)
$$

This relation suggests that a cycle of period 2 will appear if old traders are sufficiently risk averse. As a matter of fact, the reader will verify by himself that in the case of a constant risk aversion - i.e. $v_{\tau}\left(a_{\tau}\right)=a^{1-\alpha} \tau /\left(1-\alpha_{\tau}\right)$ and thus $R_{\tau}\left(a_{\tau}\right)=\alpha_{\tau}-$ then $(4.10)$ is satisfied if the old trader's risk aversion is sufficiently large, in the case in which $\ell_{2}^{*}<1, \ell_{2}^{*}<l_{1}^{*}$ and $\ell_{1}^{*}+l_{2}^{*}>1$ (Hint : find bounds for $z_{1}(1)$ and $\left.z_{2}(1)\right)$. 
We have seen once again in the course of the previous argument that cycles of period 2 were bound to coexist with the unique stationary monetary equilibrium $\theta=1$. One may thus expect that cycles displaying different periods will coexist. It turns out that one can be much more precise about this coexistence if the map $\varphi$ is unimodal, that is if $(1 . e)$ holds. Consider the following ordering of the positive integers

$$
\mid \begin{aligned}
& 3>5>7>\ldots \\
& >2 \cdot 3>2 \cdot 5>2 \cdot 7>\ldots \\
& \\
& \ldots \\
& >2^{n} \cdot 3>2^{n} \cdot 5>2^{n} \cdot 7>\ldots \\
& \ldots \\
& >\ldots>2^{m}>\ldots>8>4>2>1
\end{aligned}
$$

That is, first the odd integers greater than or equal to 3 , then the powers of 2 times these odd integers, and then the powers of 2 backward.

Given two positive integers $k$ and $k^{\prime}$, we shall note $k>_{s} k^{\prime}$ the fact that $k$ is greater than $k^{\prime}$ in the sense of the above ordering. We then have ${ }^{1}$.

THEOREM 4.3. Assume $(1 . a),(1 . c),(1 . d)$ and $(1 . e)$. Then, if the $\operatorname{map} \varphi$ has a cycle of period $k$, it has also a cycle of period $k$ ' for every positive integer $k^{\prime} s k$.

The proof of this result, which is a direct application of Sarkovskii's beautiful theorem, is given in the Appendix. 
Some implications of this very strong result are worth to be noted. It shows in particular that cycles with a period $2^{m}, m=0,1,2, \ldots$ must "appear first" before we have any hope to get a cycle with an odd period. Moreover, if there is a cycle of period 3, there is also a periodic equilibrium with period $n$, for every positive integer $n$.

In the light of the preceding result, it is interesting to investigate the circumstances implying the existence of a cycle of period 3 . Such a cycle is represented by its orbit $\left(\theta_{1}^{*}, \theta_{2}^{*}, \theta_{3}^{*}\right)$ in which each $\theta_{i}^{*}$ is a fixed point of $\varphi^{3}, \theta_{2}^{*}=\varphi\left(\theta_{1}^{*}\right) \neq \theta_{1}^{*}$ and $\theta_{3}^{*}=\varphi^{2}\left(\theta_{1}^{*}\right) \neq \theta_{1}^{*}$. It is clear here again that a cycle of period 3 is characterized by a fixed point $\theta>\bar{\theta}$ of $\varphi^{3}$ that differs from 1 - the corresponding orbit is then described by $\left(\theta, \varphi(\theta), \varphi^{2}(\theta)\right)$. Now the function $\varphi^{3}$ maps $[\bar{\theta},+\infty)$ into $[\bar{\theta}, \overline{\bar{\theta}})$ and is continuously differentiable. One has $\varphi^{3}(\bar{\theta})=\bar{\theta}, D \varphi^{3}(\bar{\theta})$ is equal to $(1 / \bar{\theta})^{3}>1$ and $\varphi^{3}(1)=1$. One cannot apply here however the argument that we employed for the case of a cycle of period 2, since

$$
D \varphi^{3}(1)=\left[\varphi^{\prime}(1)\right]^{3}<1
$$

But we can state that under assumptions $(1 . a),(1 . c),(1 . d)$, a subficient condition for the occurrence of a cycle of period 3 is that there exists $\theta$ such that $\bar{\theta}<\theta<1$ and $\varphi^{3}(\theta) \leqq \theta$. Indeed $D \varphi^{3}(\bar{\theta})>1$ implies $\varphi^{3}(\theta)>\theta$ when $\theta$ differs from but is close enough to $\bar{\theta}$, and the result follows by continuity ${ }^{l}$. We may then apply the foregoing condition,

The condition is also necessary. For otherwise the orbit $\left(\theta_{1}^{*}, \theta_{2}^{*}, \theta_{3}^{*}\right)$ of the cycle would satisfy $\theta_{i}^{*}>1$ and thus $\theta_{i}^{*}>\varphi\left(\theta_{j}^{*}\right)$. That would imply $\theta_{1}^{*}>\theta_{2}^{*}>\theta_{3}^{*}>\varphi\left(\theta_{3}^{*}\right)$, a contradiction. The same reasoning shows that under $(1 . a),(1 . c),(1 . d)$, a necessary and sufficient condition for the existence of a cycle of period 3 is that $\varphi^{3}(\theta) \geqq \theta$ for some $\theta>1$. The reader will note incidentally that the map $\varphi$ must have generically an even number of cycles of period 3 . This comes from the fact that, generically, the number of solutions of $\varphi^{3}(\theta)=\theta^{*}$ in $(\bar{\theta},+\infty)$ must be odd. 
under the simplifying assumption that $\varphi$ is unimodal, to its critical point $\theta^{*}$. Thus, under the additional assumptions $(1 . e)$ and $\alpha_{2}=\operatorname{Sup} R_{2}\left(a_{2}\right)>1$, a cycle of periad 3 will exist provided that $\theta^{*}<1$ and $\varphi^{3}\left(\theta^{*}\right) \leqq \theta^{*}$ (we know from lemma 4.2 that $\theta^{*}<1$ is then a necessary condition for the occurrence of any nondegenerate cycle). It is worth to note that under such a circumstance, one has necessarily

$$
\varphi^{2}\left(\theta^{*}\right)<\varphi^{3}\left(\theta^{*}\right) \leqq \theta^{*}<1<\varphi\left(\theta^{*}\right)
$$

Indeed we know from lemma $4.1,4)$ that $\theta^{*}<1$ implies $\varphi^{2}\left(\theta^{*}\right)<1<\varphi\left(\theta^{*}\right)$, in which case $\varphi^{2}\left(\theta^{*}\right)<\varphi^{3}\left(\theta^{*}\right)$ holds also from 1$)$ of the same lemma.

What we just said for the map $\varphi$ is of course valid for any of its topological conjugates, and thus for $x=v_{1}^{-1} \circ v_{2}$ : under the above assumptions $(1 . a),(1 . c),(1 . d),(1 . e)$ and $\alpha_{2}>1$, a cycle of period 3 occurs provided that $x\left(\mu^{*}\right)>\mu^{*}$ and $x^{3}\left(\mu^{*}\right) \leqq \mu^{*}$. In that case we have also of course

$$
\chi^{2}\left(\mu^{*}\right)<x^{3}\left(\mu^{*}\right) \leqq \mu^{*}<z_{2}(1)<\chi\left(\mu^{*}\right)
$$

Figure 5.a describes a case in which the phenomenon obtains. The central feature of the example is, as one might have expected, that the curve $x$ displays an important "hump" (this statement has an obvious counterpart for a trader's offer curve since it is isometric to the curve $x$ - see footnote 1, p.49). Fig. 5.b represents the implications of the example for the respective shapes of the curves corresponding to the functions $v_{1}$ and $v_{2}$ (the Figure shows incidentally also how the dynamics associated to the equation $v_{1}(x(\mu))=v_{2}(\mu)$ may be described with the help of the curves $v_{1}$ and $\left.v_{2}\right)$. Again, what is important is that $v_{2}$ 
displays a large hump, so that $v_{2}\left(\mu^{*}\right)$ is "large" while $v_{2}\left(x\left(\mu^{*}\right)\right)$ is "smal1",

Fig. 5.a

Fig. 5.b

We wish now to translate these heuristic statements into a formal condition that involves only the traders' utility functions, or equivalently the functions $v_{1}$ and $v_{2}$. Let us assume accordingly (1.a), (1.c), (1.d). Then under assumption (1.e) and $a_{2}>1$, the function $v_{2}$ has a unique maximum which occurs at the critical point $\mu^{*}$ of $x=v_{1}^{-1} \circ v_{2}$. The condition $\mu^{*}<x\left(\mu^{*}\right)$ means in effect that $v_{2}\left(\mu^{*}\right)>v_{1}\left(\mu^{*}\right)$. Since $v_{1}$ is increasing, the inequality $\mu^{*}<x\left(\mu^{*}\right)$ will thus obtain if and only if there exists $\bar{\mu} \geqq \mu^{*}$ such that $v_{2}\left(\mu^{*}\right)>v_{1}(\bar{\mu})$. Pick up now such a $\bar{\mu}$. Since we have $\mu^{*} \leqq \bar{\mu}<\chi\left(\mu^{*}\right)$ and since $\chi^{\prime}(\mu)<0$ for all $\mu>\mu^{*}$ we get that $x^{2}\left(\mu^{*}\right)<\chi(\bar{\mu})$. On the other hand, since the curve $x$ Ties below its tangent at $\mu=0$, i.e. $x(\mu)<\mu / \bar{\theta}$ for all $\mu>0$, we have

$$
x^{3}\left(\mu^{*}\right)<x^{2}\left(\mu^{*}\right) / \bar{\theta}<x(\bar{\mu}) / \bar{\theta}
$$

The condition $x^{3}\left(\mu^{*}\right) \leqq \mu^{*}$ will obtain accordingly (in fact with a strict inequality sign) if

$$
x(\bar{\mu})=\left(v_{1}^{-1} \circ v_{2}\right)(\bar{\mu}) \leqq \bar{\theta} \mu *
$$

or equivalently if

$$
v_{2}(\bar{\mu}) \leqq v_{1}\left(\bar{\theta}_{\mu}^{*}\right)
$$

Since the curve representing the function $v_{1}$ must lie above its tangent at the origin (see $(4.8)$ ), we have $v_{1}\left(\bar{\theta}_{\mu}{ }^{*}\right)>v_{1}\left(l_{1}^{*}\right) \bar{\theta}_{\mu}{ }^{*}$, and thus (4.12) will automatically verified if

This follows directly from (4.4) and the fact that $z_{2}^{-1}(\mu)>\bar{\theta}$ whenever $\mu>0$. One can also argue directly that the curve $x$ is isometric to the trader's offer curve. See Footnote 1 p. 49. 

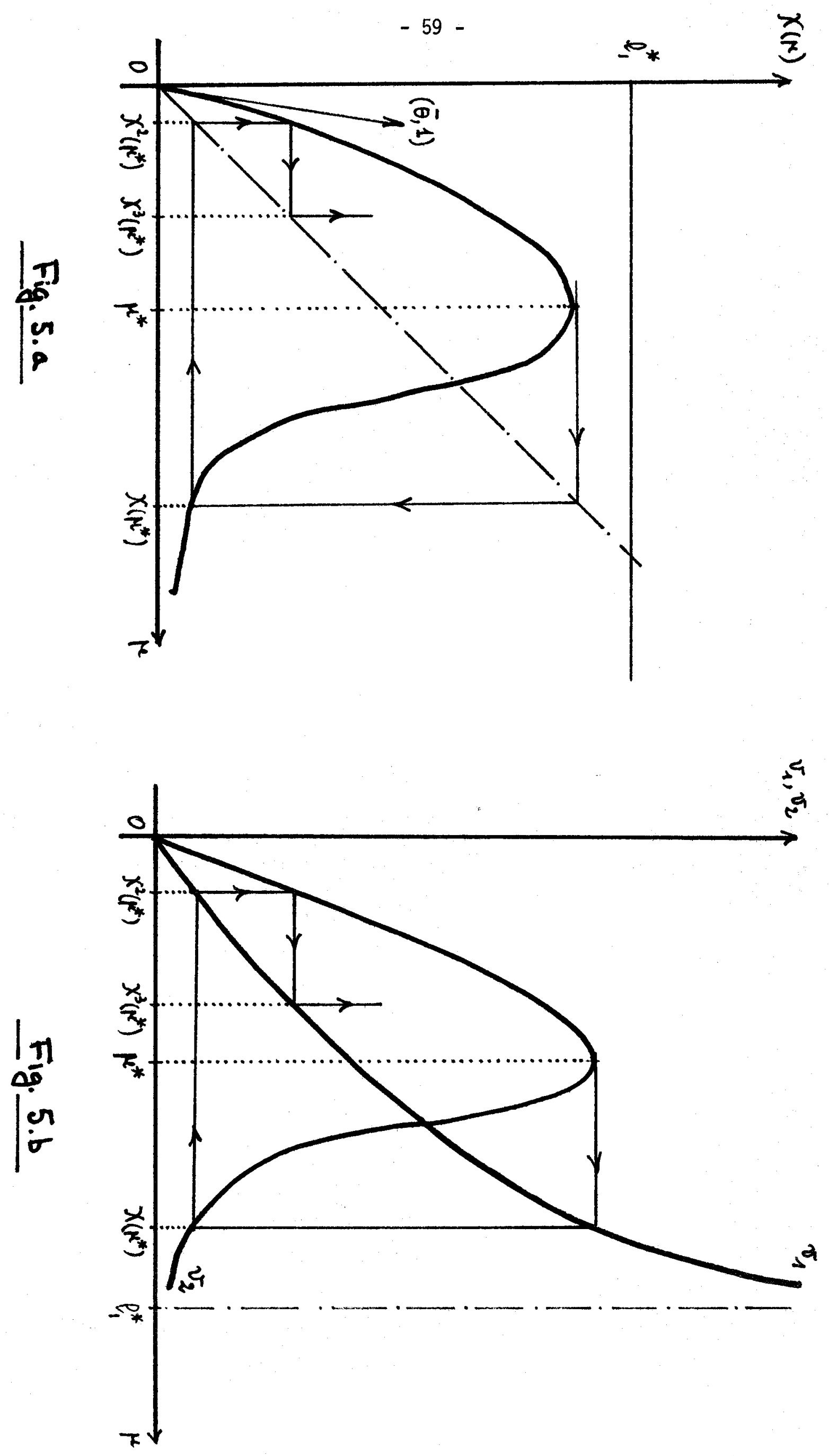


$$
v_{2}(\bar{\mu}) \leqq v_{1}\left(\ell_{1}^{*}\right) \bar{\theta} \mu^{*}
$$

To sum up, we have obtained ${ }^{1}$

PROPOSITION 4.4. Assume $(1 . a),(1 . c),(1 . d),(1 . e)$, and $\alpha_{2}=\operatorname{Sup} R_{2}\left(a_{2}\right)>1$. Let $v_{1}$ and $v_{2}$ be defined by (4.7) and eet $\mu^{*}$ be the unique maximum of $v_{2}$. Assume that there exists $\bar{\mu} \geqq \mu^{*}$ such that $v_{2}\left(\mu^{*}\right)>v_{1}(\bar{\mu})$, that satisfies $v_{2}(\bar{\mu}) \leqq v_{1}\left(\bar{\theta}_{\mu}{ }^{*}\right)$ or the stronger condition $v_{2}(\bar{\mu}) \leqq V_{1}\left(l_{1}^{*}\right) \bar{\theta}_{\mu}^{*}$. Then one has

$$
\chi^{2}\left(\mu^{*}\right)<\chi^{3}\left(\mu^{*}\right)<\mu^{*}<\chi\left(\mu^{*}\right)
$$

and there exists a cycle of period 3 .

Apart from providing a criterion to verify whether a cycle of period 3 exists, the foregoing Proposition gives a way to "generate" utility functions that entail the appearance of a cycle of period 3. Choose $\mu^{*}$ and $\bar{\mu}$ such that $\mu^{*}<\bar{\mu}<\ell_{1}^{*}$. We keep then fixed all the characteristics of the model, except the old trader's utility function $v_{2}$ (or $v_{2}$ ) which we shall vary subject to the restriction that 17 .e) holds, and that $v_{2}$ reaches $i$ ts maximum at $\mu^{*}$. Choose a value of $v_{2}^{\prime}\left(\ell_{2}^{*}+\mu^{*}\right)$ that is sufficiently high to satisfy

$$
\mu^{*} v_{2}^{\prime}\left(l_{2}^{*+\mu^{*}}\right)>\bar{j} V_{1}\left(l_{1}^{*}-\bar{\mu}\right)
$$

The conditions that we get for the existence of a cycle of period 3 are closely related to the conditions obtained by Benhabib and Day (1982) in their study of the occurrence of "chaotic" behaviour in an overlapping generations model.

2 The same statement could have been made of course by using $\varphi$ and its critical point $\theta^{*}$. 
and pick an arbitrary value of $v_{2}^{\prime}\left(l_{2}^{*}\right)\left(>V_{2}^{\prime}\left(l_{2}^{*}+\mu^{*}\right)\right)$. Then it suffices to impose a value of $V_{2}^{\prime}\left(\ell_{2}^{*}+\bar{\mu}\right)$ that is sufficiently low to satisfy $(4,13)$. The only restriction is in fact to fulfill assumption (1.e).

The next result confirms the intuition that we had when studying the occurrence of cycles of period 2, namely that the more a trader's relative degree of risk aversion increases as he gets older, the more likely is the emergence of cycles.

COROLLARY. Consider the case of a constant relative degree of risk aversion, that is $V_{\tau}\left(a_{\tau}\right)=a_{\tau}^{\left(1-\alpha_{\tau}\right)} /\left(1-\alpha_{\tau}\right)$ in which case $R_{\tau}\left(a_{\tau}\right)=\alpha_{\tau}>0$. Assume $0<\ell_{2}^{*}<1$ and keep fixed all the characteristics of the model except the old trader's relative risk aversion $\alpha_{2}$ that is free to vary. Then if $\ell_{1}^{*}+\ell_{2}^{*}>\left(1 / \ell_{2}^{*}\right)$, the assumptions of Proposition 4.4 are verified - and a cycle of period 3 thus exists - when $\alpha_{2}$ is large enough.

The proof of this assertion uses only elementary algebra. In the particular case under consideration, we have

$$
v_{1}(\mu)=\mu\left(\ell_{1}^{*}-\mu\right)^{-\alpha} 1 \text { and } v_{2}\left(\mu, \alpha_{2}\right)=\mu\left(\ell_{2}^{*}+\mu\right)^{-\alpha_{2}}
$$

We have then $\bar{\theta}\left(\alpha_{2}\right)=\left(\ell_{2}^{*}\right)^{\alpha_{2}} /\left(\ell_{1}^{*}\right)^{\alpha_{1}}$ which is a decreasing function of $\alpha_{2}$ and goes to 0 as $\alpha_{2}$ tends to infinity, since $\ell_{2}^{*}<1$. Remark next that when $\mu$ is fixed and $\alpha_{2}$ diverges to infinity, $v_{2}\left(\mu, \alpha_{2}\right)$ tends to infinity when $0<\mu<1-\ell_{2}^{*}$, to 0 when $\mu>1-\ell_{2}^{*}$, and that $v_{2}\left(1-\ell_{2}^{\star}, \alpha_{2}\right)=1-\ell_{2}^{\star}$ for all $\alpha_{2}$. On the other hand, when $\alpha_{2}>1$, the maximum of $v_{2}$ with respect to $\mu$ occurs at $\mu^{*}\left(\alpha_{2}\right)=l_{2}^{*} /\left(\alpha_{2}-1\right)$, which is a decreasing function of $\alpha_{2}$ and goes to 0 as $\alpha_{2}$ tends to infinity. It is not difficult then to verify that the maximum of $v_{2}$, that is $v_{2}\left(\mu^{*}\left(\alpha_{2}\right), \alpha_{2}\right)$, diverges to infinity when $\alpha_{2}$ goes to $+\infty$. 
If we have $l_{1}^{*}+l_{2}^{*}>\left(1 / l_{2}^{*}\right)$, then $l_{1}^{*}+l_{2}^{*}>1$ since $l_{2}^{*}<1$. Choose now an arbitrary $\bar{j}$ such that $1-l_{2}^{*}<\bar{\mu}<\ell_{1}^{*}$. We have clearly $\mu^{*}\left(\alpha_{2}\right)<\bar{\mu}$ and $v_{2}\left(\mu^{*}\left(\alpha_{2}\right), \alpha_{2}\right)>v_{1}(\dot{\mu})$ for $\alpha_{2}$ large enough. On the other hand, (4.13) will be satisfied if one can choose if so that

$$
v_{2}\left(\bar{\mu}, \alpha_{2}\right) /\left[V_{1}^{\prime}\left(\ell_{1}^{*}\right) \bar{\theta}\left(\alpha_{2}\right) \mu^{*}\left(\alpha_{2}\right)\right] \leqq 1
$$

for $\alpha_{2}$ large enough. But it is not difficult to verify that the left hand member of this inequality goes actually to 0 when $\alpha_{2}$ tends to +o if $\bar{\mu}+l_{2}^{*}>\left(1 / l_{2}^{*}\right)$. Thus if we pick up $\bar{\mu}$ such that $1-l_{2}^{*}<\bar{\mu}<\ell_{1}^{*}$ and $\bar{\mu}+l_{2}^{*}>\left(1 / \ell_{2}^{*}\right)$ - which is always possible when $\ell_{1}^{*}+l_{2}^{*}>\left(1 / \ell_{2}^{*}\right)$ - then the assumptions of Proposition 4.4 are fulfilled when $\alpha_{2}$ is large, as claimed.

Unicity of $\varphi$-stable cycles.

Theorem 4.3 above shows that cycles of very different periods will typically coexist. The stability issue is therefore essential. We present now a condition that ensure that there exists at most one $\varphi$-stable cycle. The condition is essentially that the map $\varphi$ - or one of its topological conjugates - has a "Schwarzian derivative" (to be defined shortly) that is negative. This condition is there to guarantee that the map $\varphi$ has good "expansive" properties. It will obtain in particular, in the case of a constant degree of relative risk aversion, when traders have a degree of risk aversion less than or equal to 1 in their youth and greater than or equal to 2 when they are older. The result has obviously strong implications concerning the backward perfect foresight dynamics associated to $\varphi$. But it should be emphasized that it leaves open many possibilities concerning the actual 
course followed by the economy when agents are learning, i.e. concerning the true dynamics associated to the Walrasian short run equilibrium function $W$ or $\tilde{W}$, and this even when the traders' learning process $\psi$ satisfies assumption $(3 . h)$. Specifically, even in that case, a cycle that is $\varphi$-unstable may be stable under the dynamics described by $W$ or $\tilde{W}$.

We shall need a refinement of the definition of stability for the purpose of the present study. A cycle $\left(\theta_{k}^{*}, \ldots, \theta_{1}^{*}\right)$ of $\varphi$ will be said to be weakly stable if $D \varphi^{k}\left(\theta_{j}^{*}\right) \leqq 1$. If $\varphi$ is unimodal, the cycle will be superstable if the critical point of $\varphi$ belongs to the periodic orbit, that is if $D \varphi^{k}\left(\theta_{j}^{*}\right)=0$. The notion of $\varphi$-stability is defined of course as before.

The object of the analysis that follows is to study the conditions under which the map $\varphi$ has at most one weakly stable periodic orbit, under the assumption that $\varphi$ is unimodal. Of course, in view of Lemma 4.2 , in order to make the problem nontrivial, we need to assume, in addition to $(1 . a),(1 . c),(1 . d),(1 . e)$, that $a_{2}=\sup R_{2}\left(a_{2}\right)>1$ and that $\varphi^{\prime}(1)<0$, or equivalently that the critical point of $\varphi$ satisfies $\theta^{*}<1$.

The result that we shall present uses the notion of a "Schwarzian derivative", which we define now. If $f$ is a thrice continuously differentiable function that maps the interval $[a, b]$ of the real line into itself, then the Schwarzian derivative ${ }^{1}$ of $f$, noted $S f$, is defined for every $x$ in $[a, b]$ such that $f^{\prime} \neq 0$ by

\footnotetext{
The role of the Schwarzian derivative in the study of one-dimensional dynamical systems has been discovered by singer (1978).
} 


$$
S f=\frac{f^{\prime \prime \prime}}{f^{\prime}}-\frac{3}{2}\left(\frac{f^{\prime \prime}}{f^{\prime}}\right)^{2}
$$

Direct inspection shows that $S f=-2\left|f^{1}\right|^{1 / 2} D^{2}\left[\left|f^{\prime}\right|^{-1 / 2}\right]$. So the condition that "f has a negative Schwarzian derivative" ( $\mathrm{Sf}<0$ at every $x$ such that $f^{\prime}(x) \neq 0$ ) means that $\left|f^{\prime}\right|^{-1 / 2}$ is convex on every interval of monotony of $f$. It will be satisfied in particular if $\left|f^{\prime}\right|$ (or Log $\left|f^{\prime}\right|$ ) is concave on such intervals. But these sufficient conditions are by no means necessary. Finally the reader will note that the concavity of $f$ is neither necessary nor sufficient to guarantee Sf $<0$.

In order to use this notion, we must ensure that $\varphi$ and thus the excess demand functions $z_{1}$ and $z_{2}$ are thrice continuously differentiable. This is achieved if we reinforce (1.c) by adding to it the following condition

(4. $\left.c^{\prime}\right)$ The indirect utility functions $V_{T}$ have continuous third and fourth derivatives on $(0,+\infty)$.

It is then clear that all topological conjugates of $\varphi$ obtained through a change of variable $\tilde{\theta}=h(\theta)$ will be also thrice continuously differentiable provided that $h$ has continuous third derivatives. This will be in particular the case of the map $x$.

Then we have the following important result. 
THEOREM 4.5. Assume $(1 . a),(1 . c),(1 . d),(1 . e),\left(4 . c^{\prime}\right), \alpha_{2}=\operatorname{SupR}_{2}\left(a_{2}\right)>1$ and that the critical point of $\varphi$ satisfies $\theta^{*}<1$. Assume moreover that $\varphi$ lor one of its topological conjugates $\tilde{\varphi}$ ) has a negative Schwarzian derivative on the interval $\left[\bar{\theta}, \varphi\left(\theta^{*}\right)\right]$ lor on the corresponding interval $\left.\left[h(\bar{\theta}), h\left(\varphi\left(\theta^{*}\right)\right)\right]\right)$. Then

1) The map $\varphi$ has at most one weakly stable periodic orbit.

2) If there exists a weakly stable periodic orbit, then the critical point $\theta^{*}$ is attracted to it, that is, this periodic orbit coincides with the set of accumulation points of the sequence $\left(\varphi^{j}\left(\theta^{*}\right)\right)$.

Besides the unicity of weakly stable periodic orbits, the foregoing result gives an "experimental" way of establishing whether or not a particular map $\varphi$ has a weakly stable cycle provided that it has (itself or one of its topological conjugates) a negative Schwarzian derivative. It suffices to iterate the critical point by using a computer, to check whether or not the iterates converge and to verify that the limit cycle, if any, is indeed weakly stable. Of course, since the iterations must be stopped in practice after some time, this procedure permits to discover cycles that have a small period, but is not able to distinguish between the presence of a weakly stable cycle that has a long period and the absence of any weakly stable periodic orbit. Finally it should be emphasized that there are maps that do not have any weakly stable periodic orbit. Such maps are called aperiodic. The foregoing result tells us that a map $\varphi$ that has a negative Schwarzian derivative will be aperiodic whenever the iterates of $\theta^{*}$ do not converge or if they converge to an unstable cycle.

The proof of this statement which is a direct application of the results reported in Collet and Eckmann (1980) is given in the Appendix. 
There is an obvious difficulty in applying Theorem 4.5 as it is stated, for the negativity of the Schwarzian derivative, like any statement involving convexity is not invariant when making a (nonlinear) change of variable. We may get accordingly from a particular economic model a perfect foresight map $\varphi$ that does not satisfy $S \varphi<0$ whereas one of its topological conjugates does. In that case, Theorem 4.5 applies but the model builder may not be able to diagnose it by looking at the map $\varphi$. So it is important to have a criterion using the basic characteristics of the model (i.e. utility functions and endowments) that allows recognizing directly that Theorem 4.5 applies. A partial answer to this question is provided by the following

LEMMA 4.6. Assume $(1 . a),(1 . c),(1 . d),(1 . e),\left(4 . c^{\prime}\right), \alpha_{2}=\sup R_{2}\left(a_{2}\right)>1$, and that the critical point of $\varphi$ satisfies $\theta^{*}<1$. Consider the functions $v_{1}$ and $v_{2}$ defined in (4.7) and assume that $S v_{1}(\mu) \geqq 0$ for all $\mu$ in $\left[0, \ell_{1}^{*}\right)$ and $S v_{2}(\mu)<0$ on the interval $\left[0, x\left(\mu^{*}\right)\right]$. Then SX $<0$ on $\left[0, x\left(\mu^{*}\right)\right]$ and Theorem 4.5 applies.

In particular, the foregoing condition on $v_{1}$ and $v_{2}$ obtains in the case of constant risk aversion $-v_{\tau}\left(a_{\tau}\right)=a_{\tau}\left(1-\alpha_{\tau}\right) /\left(1-\alpha_{\tau}\right)$ and thus $R_{\tau}\left(a_{\tau}\right)=\alpha_{\tau}>0-i f 0<\alpha_{1} \leqq 1$ and $\alpha_{2} \geqq 2$.

The proof of this statement uses the fact which may be verified by direct computation that the Schwarzian derivative of the composition of two (thrice continuously differentiable) maps $f$ and $g$ is given by 


$$
S(f \circ g)(x)=S f(g(x))\left[g^{\prime}(x)\right]^{2}+S g(x)
$$

Applying (4.15) to $v_{1} \circ X=v_{2}$, we get for all $\mu \geqq 0$

$$
S v_{2}(\mu)=S v_{1}(x(\mu))\left[x^{\prime}(\mu)\right]^{2}+S x(\mu)
$$

The first part of the lemma is then immediate.

In the case of a constant relative risk aversion, one has $v_{1}(\mu)=\mu\left(\ell_{1}^{*}-\mu\right)^{-\alpha} 1$ and $v_{2}(\mu)=\mu\left(\ell_{2}^{*}+\mu\right)^{-\alpha_{2}}$. Since $v_{1}^{\prime}(\mu)>0$ for a 11 $\mu$ in $\left[0, \ell_{1}^{*}\right), S v_{1}$ is defined everywhere on that interval. Direct computation shows that $S v_{1}$ is equal to (up to an everywhere positive factor)

$$
\left(1-\alpha_{1}\right)\left[\left(1-\alpha_{1}\right)\left(2-\alpha_{1}\right) \mu^{2}-4 \ell_{1}^{*}\left(2-\alpha_{1}\right) \mu+6\left(\ell_{1}^{*}\right)^{2}\right]
$$

The expressions between the brackets is easily seen to be a monotone function of $\mu$ on the interval $\left[0, \ell_{1}^{*}\right)$, which is positive on this interval. Thus $S v_{1}>0$ on $\left[0, \ell_{1}^{*}\right)$ if and only if $\alpha_{1}<1$. As for $v_{2}, S v_{2}$ is defined for every $\mu \neq \mu^{*}$ on $[0,+\infty)$ and the same sort of computation shows that it has the same sign as

$$
-\left(\alpha_{2}-1\right)\left[\left(\alpha_{2}-1\right)\left(\alpha_{2}-2\right) \mu^{2}-4 \ell_{2}^{*}\left(\alpha_{2}-2\right) \mu+6\left(\ell_{2}^{*}\right)^{2}\right]
$$

It is not difficult to verify that the expression between the brackets is positive for every $\mu \geqq 0$ when $\alpha_{2} \geqq 2$, which shows the Lemma. 
Remark. Under the assumptions of Theorem 4.5, it is possible to show that if the dynamical system $\varphi$ has a weakly stable periodic orbit, then the set of points that are not attracted to it is "exceptional", i,e. has Lebesgue measure zero (this follows from Collet and Eckmann (1980, Proposition II.5.7)). We do not insist on this otherwise nice result for the backward perfect foresight dynamics $\varphi$ has no clear meaning in the present context, beyond the fact that $\varphi$-stability implies stability in the "true" dynamics with learning under assumption (3.h). We mention the result nonetheless since it shows that some claims that "period three implies chaos" are generally unwarranted. In particular, Benhabib and Day $(1981,1982)$, Day $(1982,1983)$ use a result of Li and Yorke (1975), or a variant of it, to exhibit, under the assumption that there is a cycle of period 3, a "chaotic set", that is a set such that any perfect foresight trajectory starting from it becomes eventually erratic. This discussion shows the limits of such a statement. For if there is a weakly stable periodic orbit, the "chaotic set" - of which the existence is rightly asserted - may well be of Lebesgue measure zero, and erratic behaviour may thus be essentially unobservable - see also the remarks in Collet and Eckmann (1980, p. 20).

Bifurcation of stable periodic equilibria.

We consider now the following experiment. Suppose that we take a "onedimensional" family of economies. That is, we index the characteristics of the economy by a real number and then move this parameter over the real line. Sarkovskii's theorem suggests that the emergence of cycles as the parameter moves on should display some 
regularities, We show below that it is indeed the case for stable cycles, and that these regularities are in particular yery strong for those stable cycles that should "appear first" according to the Sarkovskii's ordering $(4,11)$, namely cycles that have a period equal to a power of 2 . Finally, we shall report on a computer experiment that was done in the case of a constant relative risk aversion, in which the old traders' risk aversion is varied - in fact increased. The result of this experiment is a "period doubling" bifurcation scheme very much alike the diagrams that are traditional in the analysis of nonlinear onedimensional dynamical systems. It shows furthermore that cycles do appear for values of the degrees of risk aversion that seem plausible, i.e. that are compatible with recent estimates of these parameters.

In order to go on we must make precise what we mean by a loneparameter) family of economies. Let us index the characteristics of the economy (the endowments $\ell_{\tau}^{*}$ and the indirect utility functions $v_{\tau}$ ) by some parameter $\lambda$, which will be taken as a real number that belongs to say, the interval $[0,1]$. The result of this indexation is denoted $\ell_{\tau \lambda}^{\star}$ and $v_{\tau \lambda}\left(a_{\tau}\right), \tau=1,2$. We assume of course that for each $\lambda$ the characteristics of the economy satisfy assumptions $(1 . a),(1 . c),(1 . d)$, and in order to make the problem nontrivial, also assumption (1.e) as well as $\alpha_{2 \lambda}=\sup _{a_{2}} R_{2 \lambda}\left(a_{2}\right)>1$. The corresponding backward perfect foresight map $\varphi_{\lambda}$ has then a unique critical point for each $\lambda$, say $\theta_{\lambda}^{*}$, and we shall postulate that $\theta_{\lambda}^{*}<1$ for all $\lambda$ (again, this condition is necessary in view of Lemma 4.2 to get nondegenerate cycles). 
We shall say finally that the family is continuous if in addition to these assumptions, the endowments $\ell_{\tau \lambda}^{*}$ depend continuousiy on $\lambda$ and if $V_{\tau \lambda}^{\prime}\left(a_{\tau}\right)$ as we11 as $V_{\tau \lambda}^{\prime \prime}\left(a_{\tau}\right)$ are jointly continuous in $\left(a_{\tau}, \lambda\right)$ for $\tau=1,2$.

In order to state the results most clearly, it will be convenient to consider a family of economies that is "rich" enough. We shall say accordingly that the family is full if it satisfies the two following conditions

1. When $\lambda=0$, the second interate of the critical point $\theta_{0}^{*}$ is such that $\theta_{0}^{*}<\varphi_{0}^{2}\left(\theta_{0}^{*}\right)$. In other words, since from Lemma $4.1,4)$ we have already $\varphi_{0}^{2}\left({ }_{0}^{*}\right)<1<\varphi_{0}\left(\theta_{0}^{*}\right)$, this condition means that the trajectory of the critical point $\theta_{0}^{*}$ begins by an oscillation around the stationary equilibrium $\theta=1$. It can be shown that under this condition, then if the map $\varphi_{0}$ has a nondegenerate cycle it must have a period at most equal to $2^{1}$.

2. When $\lambda=1$, the map $\varphi_{1}$ satisfies for instance the assumptions of Proposition 4.4, so that the trajectory of the critical point $\theta_{i}^{*}$ fulfills

$$
\varphi_{1}^{2}\left(\theta_{1}^{*}\right)<\varphi_{1}^{3}\left(\theta_{1}^{*}\right)<\theta_{1}^{*}<\varphi_{1}\left(\theta_{1}^{*}\right)
$$

and there exists a cycle of period 3 .

For details, see Collet and Eckmann (1980), especially Lemma II,2.12. 
The following result gives some insight about the fashion in which stable cycles emerge when the index $\lambda$ of a continuous full family of economies moves from 0 to 1 . First, superstable cycles of all periods $k \geqq 2$ do occur for some values of the parameter $\lambda$. Of course, given a value of $\lambda$ for which a superstable cycle obtains, a stable cycle of the same period should still exist by continuity when the characteristics of the economy are changed only a little. Second, stable periods that are equal to a power of 2 appear first and in a consecutive manner, in conformity to the ordering (4.11) of the integers. So if the family is "hice", one should observe a neat pattern of "period doubling" bifurcations, a cycle of period $2^{j}$ giving rise to a cycle of period $2^{j+1}$, and so on. Such a monotonic pattern is not general however (think of a nonmonotonic reparametrization of the family) and the sequence of stable periods $2^{j}$ may be "visited" consecutively but in a nonmonotonic fashion as the index $\lambda$ increases.

Nevertheless, the general scheme is that the sequence of values $\lambda_{j}^{*}$ for which a superstable cycle of period $2^{j}$ appears first, increases and converges to some value $\lambda_{\infty}^{*}<1$. Thus period doubling bifurcations intervene more and more rapidly as $\lambda$ moves closer to $\lambda_{\infty}^{*}$. The nice point is that if for each $\lambda$ the map $\varphi_{\lambda}$ (or one of its topological conjugates) has a negative Schwarzian derivative as in Theorem 4.5, then there exists a unique weakly stable cycle - the period of which is a power of 2 - for every $\lambda<\lambda_{\infty}^{*}$. In that case weakly stable cycles with a period that differs from a power of 2 must obtain only in the interval $\left(\lambda_{\infty}^{*}, 1\right]$. In fact a lot of phenomena may occur in that region. In particular there is an uncountable set of values of $\lambda$ in $\left(\lambda_{\infty}^{*}, 1\right]$ for which the $\operatorname{map} \varphi_{\lambda}$ has no weakly stable periodic orbit. 
Formally, we have

THEOREM 4.7. Consider a full continuous family of economies.

1) Given an arbitrary integer $k \geqq 2$, the set of parameters $\lambda$ for which the map $\varphi_{\lambda}$ has a superstable cycle of period $k$ is closed and nonempty. Given such $a \lambda$, there is an open interval around $\lambda$ such that $\varphi_{\lambda^{\prime}}$ has a stable cycle of period $k$ for all $\lambda^{\prime}$ in the interval.

2) Let $\lambda_{j}^{*}$ be the first value of the parameter $\lambda$ for which a superstable cycle of period $2^{j}$ obtains for $j \geqq 1^{2}$. Then the sequence $\lambda_{j}^{*}$ increases with $j$ and converges to some value $\lambda_{\infty}^{*}<1$ as $j$ tends to $+\infty$. For each $\lambda$ in $\left[0, \lambda_{\infty}^{*}\right)$, all cycles of the map $\varphi_{\lambda}$ have a period that is a power of 2 or are fixed points. The critical point $\theta_{\lambda}^{*}$ of $\varphi_{\lambda}$ is attracted to one of these.

3) If superstable cycles of periods $2^{j}$ and $2^{j^{\prime}}$ with $j^{\prime}>j+1$ occur respectively for the values $\lambda$ and $\lambda^{\prime}$ in $\left[0, \lambda_{\infty}^{*}\right)$, then a superstable cycle of period $2^{j}$ with $j^{\prime}>i>j$ must appear for some value in the open interval determined by $\lambda$ and $\lambda^{\prime}$.

4) Assume that each $\varphi_{\lambda}$ (or one of its topological conjugates) satisfies (4.C") and has a negative Schwarzian derivative. Then far every $\lambda$ in $\left[0, \lambda_{\infty}^{*}\right), \varphi_{\lambda}$ has a (unique) weakly stable periodic orbit.

5) Under the assumptions of 4), there exists an uncountable set of values of $\lambda$ in $\left(\lambda_{\infty}^{*}, 1\right]$ such that $\varphi_{\lambda}$ has no weakly stable cycle.

The proof of this theorem which is a direct application of the results reported in Collet and Eckmann (1980) is given in the Appendix. There are a lot of other nice results on the regularities of the bifurcations of a unimodal map $\varphi$ which are beyond the scope of this paper. For more information, see Collet and Eckmann.

That is $\lambda_{j}^{*}=\operatorname{Min}\left\{\lambda \mid \varphi_{\lambda} \quad\right.$ has a superstable cycle of period $\left.2^{j}\right\}$. 
The foregoing result suggest that one should observe first regular period doubling bifurcations in a onedimensional full continuous family of economies on some interval, and then that chaotic behaviour as well as stable cycles with a period that differ from a power of 2 should obtain later, perhaps in a mixed way. This is in fact the pattern that is always observed in computer simulations of onedimensional dynamical systems (see May (1976), or Collet and Eckmann (1980, p. 26)).

The following diagram (Fig. 6) shows the result of a similar computer experiment ${ }^{1}$ implemented in our context in the case of a constant relative degree of risk aversion - i.e. $V_{\tau}\left(a_{\tau}\right)=a_{\tau}\left(1-\alpha_{\tau}\right) /\left(1-\alpha_{\tau}\right)$ and $R_{\tau}\left(a_{\tau}\right)=\alpha_{\tau}>0$ - in which the parameter indexing the family has been taken to be the old traders' relative risk aversion $\alpha_{2}$. Then we know from Lemma 4.6 that if $\alpha_{1} \leqq 1$ and $\alpha_{2} \geqq 2$, Theorem 4.5 applies. In that case, it suffices to iterate the critical point $\theta^{*}$ sufficiently long to discover the unique weakly stable cycle whenever it exists.

This procedure was applied in fact to the map $x$ that describes the backward perfect foresight dynamics of equilibrium real balances. The parameter $\alpha_{2}$ was made to vary between 2 and 16 by steps of 0.05 .

The computer experiment has been in fact run by Rose-Anne Dana and Dominique Lévy, whose help is gratefulty acknowledged. Earlier computer simulations by Alain Morineau were also very valuable. 
For each such value of $\alpha_{2}$ on the horizontal axis, the corresponding map $x$ was iterated 300 times, the initial point being the critical point of the map, which is in that case $\mu^{*}=l_{2}^{*} /\left(\alpha_{2}-1\right)$. On the vertical axis above $\alpha_{2}$ were plotted all the values of the iterated equilibrium real balances $\mu_{t}$ from $t=200$ to $t=300$. With this procedure, one may hope the figure to show clearly the weakly stable cycles that do no have too long a period and that are attractive enough. The experiment cannot of course discriminate between stable cycles that have a long period, and erratic behaviour.

The result of the experiment is shown below in Fig. 6, under the specification $\alpha_{1}=0.5, \ell_{1}^{*}=2$ and $\ell_{2}^{*}=0.5$. It shows indeed the usual period doubling bifurcation pattern. One may note moreover that cycles do emerge for values of the relative degrees of risk aversion that do not seem implausible. Indeed, the limit value of the period doubling bifurcations (the analogue of $\lambda_{\infty}^{*}$ in Theorem 4.7 ) is about 8 , while recent estimates of the Arrow-Pratt measure of risk aversion which are averages over a whole population - range about 6 . $^{1}$

\section{Fig. 6}

See I. Friend and J. Hasbrouck (1981). I wish to thank Albert Ando, to whom I owe this reference. 


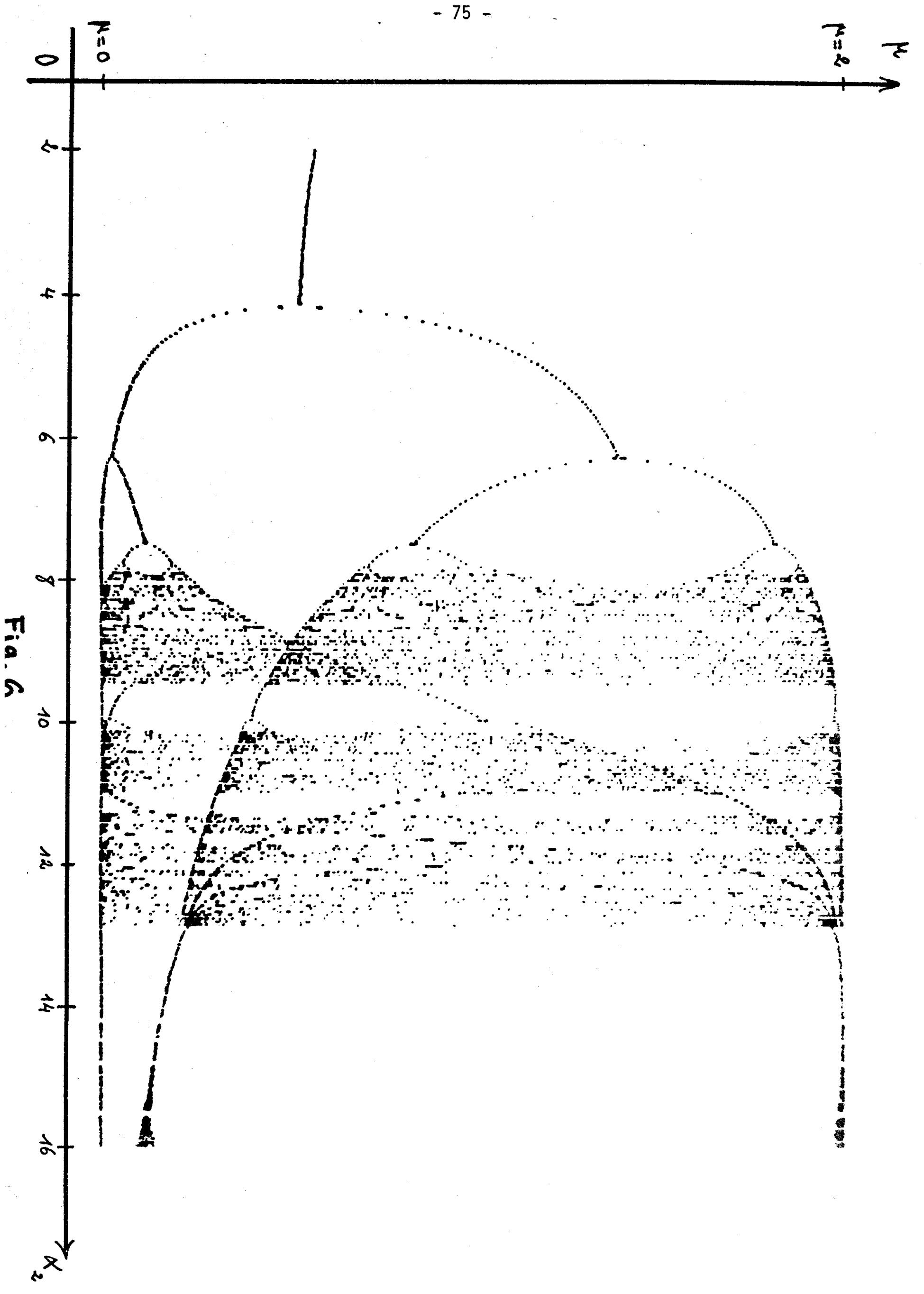


5. OUTPUT AND ANTICIPATED (OR UNANTICIPATED) REAL INTEREST RATES.

We were concerned in the previous Sections with the existence and stability of periodic competitive equilibria. We wish now to study the following issue. Since there are movements of output (or equivalently of the rate of participation of the labour force) and of prices along a sequence of competitive equilibria, is there any systematic relationship between the equilibrium levels of output and of the real rate of interest ? It will be shown that such a relation exists indeed in the present model, both in the short run and in the long run (i.e. along periodic equilibria). Of course, since no interest is paid on money in the model, this implies a relation between equilibrium output and inflation that goes simply in the opposite direction. It will be seen moreover that high levels of output are associated to low real interest rates (i.e. to high levels of inflation) whenever old traders have a higher marginal propensity to consume leisure than young ccnsumers. Output and inflation are then "procyclical". Furthermore, it will be shown that such an association holds for anticipated as well as unanticipated movements of the real interest rate (or the rate of inflation).

We first begin with long run, i.e, periodic, equilibria. Let us assume that the economy satisfies assumptions $(1 . a),(1 . b)$, (1.c), and consider a periodic competitive equilibrium $\left(p_{t}\right)$ with period $k$. We know from Proposition 2.3 that under assumption $(2.9)$, the sequence verifies for all $t$

$$
z_{1}\left(\theta_{t}\right)+z_{2}\left(\theta_{t-1}\right)=0
$$




$$
M / p_{t}=z_{2}\left(\theta_{t-1}\right)
$$

in which $\theta_{t}=p_{t} / p_{t+1}$. Moreover one has $\bar{\theta}<\theta_{t}<\overline{\bar{\theta}}=z_{2}^{-1}\left(\ell_{1}^{*}\right)$ for all $t$.

The above relations describe the evolution of the real interest rates $\rho_{t}=\theta_{t}-1$ over the cycle. It is then straightforward to deduce the corresponding evolution of equitibrium output. The sequence of real equilibrium money stocks $\mu_{t}=M / p_{t}$ satisfies $0<\mu_{t}<\ell_{1}^{*}$ for all $t$. At date $t$, the young trader's equilibrium consumption and labour supply are then obtained by maximizing $U_{1}\left(c_{1}, \ell_{1}^{*}-l_{1}\right)$ under the budget constraint $\left[c_{1}+\left(\ell_{1}^{*}-\ell_{1}\right)\right]=\ell_{1}^{*}-\mu_{t}$. In view of problem $(1.7)$, the outcome is $c_{1}\left(\ell_{1}^{*}-\mu_{t}\right)$ and $\ell_{1}\left(\ell_{1}^{*}-\mu_{t}\right)$. By the same kind of reasoning, the old trader's equilibrium consumption and labour supply are given by $c_{2}\left(\ell_{2}^{*}+\mu_{t}\right)$ and $\ell_{2}\left(\ell_{2}^{*}+\mu_{t}\right)$. Equilibrium output $y_{t}$ at date $t$ is therefore

$$
\begin{aligned}
y_{t} & =l_{1}\left(l_{1}^{*}-\mu_{t}\right)+l_{2}\left(l_{2}^{*}+\mu_{t}\right) \\
& =c_{1}\left(l_{1}^{*}-\mu_{t}\right)+c_{2}\left(l_{2}^{*}+\mu_{t}\right)
\end{aligned}
$$

Let us define now for every $\theta$ in the interval $[\bar{\theta}, \overline{\bar{\theta}}]$

$$
f(\theta)=\ell_{1}\left(\ell_{1}^{*}-z_{2}(\theta)\right)+\ell_{2}\left(\ell_{2}^{*}+z_{2}(\theta)\right)
$$

Then (5.2) and (5.3) imply immediately that there is a systematic relationship, along a periodic equilibrium with perfect foresight, between the equilibrium output leve $1 y_{t}$ and $\theta_{t-1}=p_{t-1} / p_{t}$ - or equivalently the rate of inflation $p_{t} / p_{t-1}$. It is indeed described by $y_{t}=f\left(\theta_{t-1}\right)$. 
One may remark that the function $f$ depends only on the real characteristics of the economy and not on the level of the money stock M. Moreover the relation between $y_{t}$ and $\theta_{t-1}$ is independent of the period $k$ of the cycle $^{1}$.

The natural question to ask then is whether a low real interest rate (i.e. a high rate of inflation) is associated to a high level of activity along a periodic competitive equilibrium with perfect foresight - or equivalently whether $f$ is decreasing. To this effect, consider an increase of $\theta_{t-1}$. Since the function $z_{2}$ is increasing, the old consumer's "real income" at $t$, i.e. $\ell_{2}^{*}+z_{2}\left(\theta_{t-1}\right)$, goes up while the young consumer's "real income" $\ell_{1}^{*}-z_{2}\left(\theta_{t-1}\right)$ goes down by the same amount. If we assume the labour supply functions $\ell_{\tau}$ to be continuously differentiable, the overall consequence of this "redistribution of income" among the two traders living at $t$ will depend upon the relative magnitudes of the marginal propensities to supply labour - or to consume leisure at both ages. This motivates considering the following assumption

As a matter of fact, we used periodicity only to ensure through assumption $(2.9)$ that expectations were correct along the cycle. Thus the relation $y_{t}=f\left(\theta_{t-1}\right)$ is valid for any (even aperiodic) sequence of temporary competitive equilibria aith perfect foresight. 
(5.i) The functions $c_{\tau}\left(a_{\tau}\right)$ and $\ell_{\tau}\left(a_{\tau}\right)$ are continuously differentiable. The good and leisure are noninferior commodities, that is, $0<c_{\tau}^{\prime}\left(a_{\tau}\right)<1$ and $-1<\ell_{\tau}^{\prime}\left(a_{\tau}\right)<0$ for every $a_{\tau} \geqq 0, \tau=1,2$. Furthermore, the marginal propensity to consume leisure is higher for an old trader than for a young one, that is, $\left|\ell_{2}^{\prime}\left(a_{2}\right)\right|>\left|\ell_{1}^{\prime}\left(a_{1}\right)\right|$ for all $a_{1}, a_{2} \geqq 0$ such that $a_{1}+a_{2}=\ell_{1}^{*}+\ell_{2}^{*}$.

It is then quite easy to see that $f$ is a decreasing function, If $\theta_{t-1}$ goes up, the old trader living at $t$, being richer, supplies less labour (consumes more leisure) white the young consumer, being poorer, works more, But assumption (5.i) makes the aggregate labour supply, and thus output, to go down. Formally, we have by differentiating $(5,4)$

$$
f^{\prime}(\theta)=z_{2}^{\prime}(\theta)\left[\ell_{2}^{\prime}\left(\ell_{2}^{*}+z_{2}(\theta)\right)-\ell_{1}^{\prime}\left(\ell_{1}^{*}-z_{2}(\theta)\right)\right]<0
$$

To sum up, we have obtained

One may note that in view of $(1.8)$, one has $c_{\tau}^{\prime}\left(a_{\tau}\right)-\ell_{\tau}^{\prime}\left(a_{\tau}\right) \equiv 1$. Differentiability of $c_{\tau}$ and $\ell_{\tau}$ could have been obtained from suitable differentiability assumptions on $U_{\tau}$, provided that "corner" solutions are avoided in (1.7), as in footnote 2, p. 12. Then the assumptions stated in $(5 . i)$ can be traced back to the appropriate conditions on the utility functions $U_{\tau}$. In particular, consumption and leisure are noninferior goods whenever the marginal rate of substitution bewteen consumption and leisure is an increasing function of leisure and a decreasing function of consumption. 
PROPOSITION 5.1. Assume $(1 . a),(1 . b),(1 . c)$, and define for any $\theta$ in $[\bar{\theta}, \overline{\bar{\theta}}]$, in which $\overline{\bar{\theta}}=z_{2}^{-1}\left(\ell_{1}^{*}\right)$,

$$
f(\theta)=\ell_{1}\left(\ell_{1}^{*}-z_{2}(\theta)\right)+\ell_{2}\left(\ell_{2}^{*}+z_{2}(\theta)\right)
$$

Then under assumption (2.9), for any sequence of competitive equilibria $\left(p_{t}\right)$ that has period $k$, equilibrium output $y_{t}$ and $\theta_{t-1}=p_{t-1} / p_{t}$ are linked by $y_{t}=f\left(\theta_{t-1}\right)$ for all $t$, Moreover, under assumption (5.i), $f$ is continuously differentiable, and $f^{\prime}(\theta)<0$.

The relationship that we just established between the equilibrium output at date $t$ and the real interest rate between dates $t-1$ and $t$, i.e. $\theta_{t-1}-1$, is clearly a "long run" relationship. That is, it obtains only when the economy has converged to a "steady state", i,e. to a (stable) periodic equitibrium, and when learning has ceased accordingly. It should be emphasized moreover, that the long ran relation $y_{t}=f\left(\theta_{t-1}\right)$ does not represent a behavioural supply or demand function, since it involves equilibrium variables over the cycle.

It is useful to have a brief look at the sort of relationship that exists between equilibrium output and the real interest rate in the short run, i.e., during the adjustment process of the economy toward long run periodic equilibria. This will enable us in particular to single out the role that "surprises" (forecasting errors) play in the short run.

Consider accordingly an arbitrary (aperiodic) sequence of temporary equilibrium prices $\left(p_{t}\right)$. As before equilibrium real balances are given by $H_{t}=M / p_{t}$, while the sequence of equilibrium output $y_{t}$ 
is given by (5.3), in which $0<\mu_{t}<\ell_{1}^{*}$. But equilibrium real balances are not given by (5.2) this time, since we are considering an aperiodic sequence along which traders are still learning. We must use instead the temporary equilibrium conditions (2.1), (2.2), or equivalently (2.3). As a matter of fact, we may rewrite $(2,3)$ for date $t-1$ as

$$
\mu_{t}=\frac{p_{t}^{e}}{p_{t-1}} \frac{p_{t-1}}{p_{t}} z_{2}\left(\frac{p_{t-1}}{p_{t}^{e}}\right)
$$

in which the price $p_{t}^{e}$ that is expected for date $t$ by the young trader living at date $t-1$ is determined by

$$
p_{t}^{e}=\psi\left(p_{t-1}, \ldots, p_{t-T-1}\right)
$$

We see then that the equilibrium real balance $\mu_{t}$ at $t$ is a function of the real interest rate that was expected in equilibrium at the date $t-1$, or more precisely of $\theta_{t-1}^{e}=p_{t-1} / p_{t}^{e}$, and of the forecasting error actually made on this variable, i.e. of $e_{t}=\theta_{t-1} / \theta_{t-1}^{e}$, in which ${ }_{t-1}=p_{t-1} / p_{t}$ (one has equivalently $e_{t}=p_{t}^{e} / p_{t}$ ). Indeed, (5.5) can be rewritten with this notation

$$
\mu_{t}=e_{t} z_{2}\left(\theta_{t-1}^{e}\right)
$$

Here again, since the nominal interest rate paid on money is zero in the model, there is an inverse relationship between the (expected) rate of inflation and the (expected) real rate of interest. In particular, a high level of "unanticipated inflation" means that the real interest rate $\theta_{t-1}$ is much lower than was expected, that is, it means a 
low value of $e_{t}$. Of course, perfect foresight corresponds to $e_{t}=1$ and thus to $\theta_{t-1}^{e}=\theta_{t-1}\left(\right.$ or $\left.p_{t}^{e}=p_{t}\right)$.

Let us define the function $F\left(\theta^{\mathrm{e}}, \mathrm{e}\right)$ by

$$
F\left(\theta^{e}, e\right)=\ell_{1}\left(l_{1}^{*}-e z_{2}\left(\theta^{e}\right)\right)+\ell_{2}\left(l_{2}^{*}+e z_{2}\left(\theta^{e}\right)\right)
$$

the domain of definition of $F$ being the set of pairs $\left(\theta^{e}, e\right)$ such that $\bar{\theta} \leqq \theta^{e} \leqq z_{2}^{-1}\left(\ell_{1}^{*} / e\right)$ in order to ensure that $0 \leqq e z_{2}\left(\theta^{e}\right) \leqq \ell_{1}^{*}$. Examination of (5.3), (5.5) and (5.6) shows then immediately that along a sequence of temporary equilibria, equilibrium output is linked to the expected real interest rate and to the forecasting error made on this variable by

$$
\begin{aligned}
& y_{t}=F\left(\theta_{t-1}^{e}, \theta_{t-1} / \theta_{t-1}^{e}\right) \text { in which } \theta_{t-1}=p_{t-1} / p_{t} \text { and } \\
& \theta_{t-1}^{e}=p_{t-1} / \psi\left(p_{t-1}, \ldots, p_{t-T-1}\right) \text {. }
\end{aligned}
$$

Two important facts are worth to be noticed. First, the function $F$ depends only on the real characteristics of the economy, and not: on the level of the money stock M. More importantly, it does not depend on the particular trajectory that is considered nor on the learning process $\psi$ that the traders are using ${ }^{1}$. This relation $F$ would be in

The relation between the equilibrium output level $y_{t}$ and equilibrium current and past prices $\left(p_{t}, \ldots, p_{t-T-1}\right)$ that is implied by $(5,9)$ depends of course on the traders' learning process $\psi$. But it does not depend either on the particular equilibrium trajectory under consideration. 
particular the same if traders employed a more sophistịcated learning procedure than the one we considered here, for instance if the expectation function (the agent's "model" of the economy) was revised according to a prespecified rule that would take into account the forecasting errors made in the past (see the Remark at the end of Section 2). Second, when $e=1$, the "short run" function $F$ reduces to the "long run" map $f$, since $F(\theta, 1) \equiv f(\theta)$. Thus if we consider a periodic equilibrium $\left(p_{t}\right)$ with perfect foresight (assumption (2.g)), then there are no forecasting errors along the sequence $\left(e_{t}=1\right.$ and $\theta_{t-1}^{e}=\theta_{t-1}$ for all $\left.t\right)$, and $(5,9)$ yields the long run relationship $y_{t}=f\left(\theta_{t-1}\right)$ that we established previously (this finding comes evidently from the fact that when there is perfect foresight, the money equilibrium equation $(2,3)$, and thus $(5.5)$, reduces to $(5,2))$.

The final step of this investigation is to examine whether a low value of $\theta_{t-1}^{e}$ (i.e. a high anticipated inflation) and/or a low value for the error forecast $e_{t}$ (i.e. a large unanticipated inflation) is associated to a large equitibrium output $y_{t}$. It is indeed immediate to verify that under assumption $(5 . i)$, both anticipated and unanticipated inflation yield a larger level of output, or equivalently, that $F$ is a decreasing function of each yariable $\theta^{e}$ and $e$. Indeed, by differentiation of $(5.8)$

$$
\begin{aligned}
& F_{\theta^{\prime}}^{\prime}=e z_{2}^{\prime}\left(\theta^{e}\right)\left[\ell_{2}^{\prime}\left(l_{2}^{*}+e z_{2}\left(\theta^{e}\right)\right)-\ell_{1}^{\prime}\left(\ell_{1}^{*}-e z_{2}\left(\theta^{e}\right)\right)\right]<0 \\
& F_{e}^{\prime}=z_{2}\left(\theta^{e}\right)\left[\ell_{2}^{\prime}\left(l_{2}^{*}+e z_{2}\left(\theta^{e}\right)\right)-\ell_{1}\left(\ell_{1}^{*}-e z_{2}\left(\theta^{e}\right)\right)\right]<0
\end{aligned}
$$


It may be noted incidentally that the ratio of the elasticities of equilibrium output with respect to $\theta^{e}$ and $e$, is equal to

$$
\left(\theta_{\theta}^{e^{\prime}} \mathrm{F}^{\prime}\right) /\left(\mathrm{eF}_{e}^{\prime}\right)=\theta^{e} z_{2}^{\prime}\left(\theta^{e}\right) / z_{2}\left(\theta^{e}\right)
$$

that is, to the elasticity of the function $z_{2}$. It will be greater than 1 if and only if the "demand for real balances" - which is equal, by virtue of $(1.3)$, to $z_{2}\left(\theta^{e}\right) / \theta^{e}$ - is increasing at $\theta^{e}$ (all what is needed to establish this property is that $F$ is differentiable, with $F_{e}^{\prime} \neq 0$. There was no need of assumption $(5 . i))^{1}$.

Finally, we should emphasize here again that the short run relationship (5.9) between output and anticipated as well as unanticipated real interest rates represents in no way any behavioural demand or supply function, as it inyolves equilibrium magnitudes. In the language of econometrics, the short run or long run relations between equilibrium output and expected or unexpected real interest rates are reduced forms, not structural forms,

To sum up, we have obtained

The fact that $F$ has all those nice simple properties should not obscure the fact that the short run relation between equilibrium output $y_{t}$ and current and past equilibrium prices $\left(p_{t}, \ldots, p_{t-T-1}\right)$ that is implied by (5.9), i.e. when taking into account the traders' learning process, may be quite complicated. 
PROPOSITION 5.2. Assume (1.a), (1.b), (1.c) and define for any positive $\left(\theta^{\mathrm{e}}, \mathrm{e}\right)$ satisfying $\bar{\theta} \leqq \theta^{\mathrm{e}} \leqq \mathrm{z}_{2}^{-1}\left(\ell_{1}^{*} / \mathrm{e}\right)$,

$$
F\left(\theta^{e}, e\right)=\ell_{1}\left(\ell_{1}^{*}-e z_{2}\left(\theta^{e}\right)\right)+\ell_{2}\left(\ell_{2}^{*}+e z_{2}\left(\theta^{e}\right)\right)
$$

Then for any sequence of temporary competitive equilibrium prices $\left(p_{t}\right)$, equilibrium output $y_{t}$ is linked to anticipated and unanticipated real interest rates by $y_{t}=F\left(\theta_{t-1}^{e}, \theta_{t-1} / \theta_{t-1}^{e}\right)$, in which $\theta_{t-1}=p_{t-1} / p_{t}$ and $\theta_{t-1}^{e}=p_{t-1} / \psi\left(p_{t-1}, \ldots, p_{t-T-1}\right)$, for all $t$. Moreover, under assumption (5.i), $F$ is continuously differentiable, and $F_{\theta}^{\prime} e 0, F_{e}^{\prime}<0$.

Remarks.

1. It has been shown incidentally in this Section that equilibrium real balances $\mu_{t}$ along a sequence of temporary competitive equilibria are positively related to the expected real interest rate $\theta_{t-1}^{e}$ and to the forecasting error on this variable $e_{t}=\theta_{t-1} / \theta_{t-1}^{e}$, see (5.7). The long run version of this relation (along periodic equilibria with perfect foresight) is (5.2). The validity of the result does not hinge on (5.i). In fact, it would hold in an exchange economy (without production) in which traders are endowed with the quantities $\ell_{1}^{*}, \ell_{2}^{*}$ of the good in each period of their lifes.

2. The fact that equilibrium real balances at $t$ depend oniy on $\theta_{t-1}^{e}$ and on $e_{t}=\theta_{t-1} / \theta_{t-1}^{e}$ along a sequence of temporary equilibria, as in (5.7), rests upon the specific structure of the model that we have considered. If traders 1 ived $n$ periods, equilibrium real balances at $t$ would depend upon the expectations and on the forecasting errors that the agents living at $t$ made in all the previous periods of their lifes. 
There would be then a lag between equilibrium real balances (or output) and expectations as well as forecasting errors, which would be equal to the length $n$ of the traders' lifetimes.

3. The long run and short run relationships $f$ and $F$ may of course be expressed in equivalent ways, by implementing a consistent change of variables. Let us transform the output variable y into $\tilde{y}$ by $\tilde{y}=h(y)$, in which $h$ is continuously differentiable and increasing $\left(h^{\prime}>0\right)$. Let us define $\tilde{\theta}=h(\theta), \widetilde{\theta}^{e}=h\left(\theta^{e}\right)$. The new forecasting error variable $\tilde{e}=h(e)$ is then related to $\tilde{\theta}$ and $\tilde{\theta}^{e}$ by

$$
\tilde{\mathrm{e}}=h(e)=h\left(\theta / \theta^{\mathrm{e}}\right)=h\left(h^{-1}(\tilde{\theta}) / h^{-1}\left(\tilde{\theta}^{\mathrm{e}}\right)\right)
$$

We may then write

$$
\begin{aligned}
\tilde{y} & =h\left[F\left(h^{-1}\left(\tilde{\theta}^{e}\right), h^{-1}(\tilde{e})\right)\right] \\
& =\tilde{F}(\tilde{\theta} \mathrm{e}, \tilde{e})
\end{aligned}
$$

and it is easily seen that the partial derivatives of $\tilde{F}$ with respect to $\tilde{\theta}^{e}$ and $\tilde{e}$ have the same sign as the partial derivatives of $F$ with respect to $\theta^{e}$ and $e$. With the new variables, perfect foresight means $\tilde{e}=h(1)$ - and thus $\tilde{\theta}^{\mathrm{e}}=\tilde{\theta}$ - and the long run relationship becomes

$$
\tilde{y}=\tilde{f}(\tilde{\theta}) \equiv \tilde{F}(\tilde{\theta}, h(1))
$$

One has in fact $\tilde{f}=$ hofoh ${ }^{-1}$, and thus the derivatives of $\tilde{f}$ and $f$ have the same sign.

The same sort of exercise may be done for the relation between rea 1 balances and expected or unexpected real interest rates. The reader may go through this change of variables when $h$ is the logarithmic function. 
In that case, the result resembles - if one excepts the fact that our model is highly nonlinear - quite a few log-linear macroeconomic models that have been so popular recently in the socalled "New Classical" macroeconomic literature. The other important differences are first that (the logarithm of) equilibrium output and real balances - or their deviations from their "permanent" values, which may be viewed as those that correspond to the Golden rule stationary state $\theta=1$ - depend not only on (the logarithm of) the "surprise" $e_{t}=\theta_{t-1} / \theta_{t-1}^{e}=p_{t}^{e} / p_{t}$ but also on the expected real interest rate $\theta_{t-1}^{e}=p_{t-1} / p_{t}$; and second that these relationships cannot be interpreted as supply or demand functions as they often are, apparentiy mistakenly, since they involve equilibrium magnitudes. 
6. STABILIZING PERIODIC EQUILIBRIA.

The business cycles that were described in the preceding Sections are purely endogenous, that is, they are not attributable to any hypothetical exogenous "shocks" such as variations of the consumers' tastes, of their endowments or of productivity, nor to changes of the Government's policy - there are none. They are not due either to a lack of information of the traders since learning has been completed along periodic equilibria with perfect foresight. We wish to show now that the Government is in fact able to stabilize completely business cycles by choosing an appropriate countercyclical policy.

There are many ways through which the Government may hope to influence economic activity : money transfers that are proportional to the agents' money balances (interest payments), lump sum transfers (taxes or subsidies), income taxes, purchases of goods, open market operations, and so on. The subject matter is obviously too vast to be dealt with in depth within the limited scope of the present paper. We shall focus attention accordingly on the simple and popular case in which the Government pays a (publicly announced) nominal rate of interest at each date on the money balances that old consumers hold. We shall then find the analogue in our framework of a standard result : if the monetary authority pegs the nominal rate of interest (or equivalently the rate of growth of the nominal money supply) at some arbitrary predetermined level, this policy will have no real effect on the set of corresponding long run (periodic) equilibria - nor on the long run "trade off" between equilibrium output (or real balances) and real interest rates that we established in the preceding Section. Moreover, if the traders believe in the neutrality 
of such a policy, it will have no real effect either on the trajectories that the economy follows during the adjustment process toward long run equilibria. It will not change then in particular the stability or the unstability of a particular periodic equilibrium. In this sense, permanent changes of the rate of growth of the money supply that are implemented through a predetermined nominal interest payment are superneutral ${ }^{1}$.

The analysis will thus confirm the analogue in this context of the "monetarist" claim, namely that any given cycle of the model is in fact compatible with an arbitrary (average) rate of inflation. Although this conclusion is correct, the fact that the foregoing policy of pegging the nominal interest rate at some predetermined level has no real effects is entirely due to the feature that such a policy is purely passive. We shall show that the monetary authority can in fact influence real events by adopting an active policy, e.g. a feedback rule that links the real rate of growth of the money supply at some date to previously observed economic variables. Note that such an active policy leaves in any case the set of "potential" long run periodic equilibria with perfect foresight invariant (in real terms). It is indeed the same as under laisser faire, i.e. it is described by the set of all cycles of the map $\varphi^{2}$.

It must be emphasized that this superneutrality result non longer holds if the rate of growth of the money supply is changed by other means, e.g. through lump sum money transfers, or Government's purchases of the good. See Remark 1 at the end of the Section.

The fact that monetary policy cannot create new cycles in real terms is specific to nominal interest payments. If changes of the money supply are brought about by lump sum transfers or Government's purchases, then policy can create new cycles or stabilize them. See Remark 1 at the end of the Section. 
What such an active policy can at best achieve is thus to "select" one particular cycle that would not have been reached under laisser faire, We shall see indeed that there is a simple, moderately countercyclical ("leaning against the wind") policy of that sort that enables the monetary authority to stabilize completely the economy (to peg the real rate of interest permanentiy at a level equal to 0 ), and to control at the same time the nominal rate of growth of the money supply (or equivalently, the rate of inflation) at some predetermined level $r^{\star}$. Of course any change of $r^{*}$ alone will be here again superneutral ${ }^{1}$. The analysis will thus bring into the forefront a phenomenon that appears general, namely that a change of the average growth rate of the money supply yields consequences that may be very different from those that result from the "transitory" part of the policy, i.e. from the active countercyclical (or procyclical) feedback rule that is implemented by the monetary authority. It should be noted also that a successful stabilization policy like the above affects not only the variances of real equilibrium magnitudes but also their means (their average over a cycle) since the model is nonlinear, in contrast to traditional macroeconomic models that use the "natural rate" hypothesis.

Before going into the details of the study, we must look at an agent's behaviour when interest is paid on money. Let us consider accordignly a young trader at a time in which the price of the good is $p$ and let $p^{e}$ and $r^{e}=x^{e}-1$ be the price of the good and the transfer (nominal interest rate) he anticipates for the next date. The agent's problem is then to maximize his

Here again this superneutrality result non longer holds if the rate of growth of the money supply is changed by other means, e.g. through lump sum money transfers, or Government's purchases of the good. See Remark 1 at the end of the Section. 
intertemporal utility function $U_{1}\left(c_{1}, l_{1}^{*}-l_{1}\right)+U_{2}\left(c_{2}, l_{2}^{*}-l_{2}\right)$ subject to the current and expected budget constraints

$$
\begin{aligned}
& p\left(c_{1}-\ell_{1}\right)+m=0 \\
& p^{e}\left(c_{2}-\ell_{2}\right)=m x^{e}
\end{aligned}
$$

It is then clear that under assumptions (1.a), (1.b), his current and future excess demands for the good are given by $z_{1}(\theta)$ and $z_{2}(\theta)$ respectively, in which $\theta=p x^{e} / p^{e}$ (again $\theta-1$ is the expected real interest rate), while his demand for money is $m^{d}\left(p, p^{e} / x^{e}\right)$.

We proceed now to the determination of the dynamical system that governs the evolution of equilibrium prices and interest rates. First note that if $x_{t}=\left(1+r_{t}\right)$ describes the transfer actually made at date $t$ and if $M_{t-1}$ is the pretransfer money stock at that date, then the money supply at $t$ is $M_{t}=x_{t} M_{t-1}$. The equilibrium conditions at date $t$ for good and money are then described by

$$
\begin{gathered}
z_{1}\left(p_{t} x_{t+1}^{e} / p_{t+1}^{e}\right)+\left(M_{t} / p_{t}\right)=0 \\
m^{d}\left(p_{t}, p_{t+1}^{e} / x_{t+1}^{e}\right)=M_{t}
\end{gathered}
$$

in which $p_{t+1}^{e}$ and $x_{t+1}^{e}$ stand for the price and the transfer that the yound trader living at $t$ anticipates for the next date. By Walras's Law, the two equations are equivalent and may be written in the following form

$$
\left(p_{t+1}^{e} / x_{t+1}^{e}\right) z_{2}\left(p_{t} x_{t+1}^{e} / p_{t+1}^{e}\right)=M_{t}
$$


These three equations are the analogues of (2.1), (2.2) and (2.3) for the no interest payment case.

To be complete, the system must involve the specification of the dynamical policy that is implemented by the monetary authority, i.e. of the way in which the transfer at date $t+1$, or $x_{t+1}=1+r_{t+1}$, depends on the price at that date $p_{t+1}$ and of the economic data that were observed by the monetary authority at the previous dates. The system must specify in addition the traders' forecasts about prices and interest rates - in fact about the ratio $p_{t+1}^{e} / x_{t+1}^{e}$ - as a function of current and past prices and rates of interest. One may note that the resulting expectation function will depend in a crucial way on whatever information the traders have concerning the particular policy that has been chosen by the monetary authority.

We shall consider exclusively in the sequel policies that aim at pegging in the long run the nominal interest rate at some predetermined level $r^{*}$, and may be some additional "real" variables. It is then useful as a first step to characterize the set of all long run periodic equilibria with perfect foresight that may arise in the economy when the interest rate is $r^{*}$, and this independently of any further specification of the government's policy or of the learning processes that the consumers employ. As we are going to see, this set coincides - as far as real equilibrium magnitudes are concerned - with the set of periodic equilibria with perfect foresight that arises in the no interest payment case, i.e. under laisser faire. The only difference is that prices grow now "on average" at the rate $r^{*}$ along the cycles. 
The argument is indeed trivial. If the interest rate is pegged at the level $r^{*}$, one has $M_{t}=x^{*} M_{t-1}$ for al1 $t$, in which $x^{*}=1+r^{*}$. Consider now a sequence of prices $\left(p_{t}\right)$ that has (primitive) period $k$ and that grows on average at the rate $r^{*}$, i.e. such that $p_{t+k}=\left(x^{*}\right)^{k} p_{t}$ for a $11 t^{1}$. Then by definition, the sequence $\left(p_{t}\right)$ is a periodic equilibrium with perfect foresight corresponding to $r^{*}$ if and only if it satisfies (6.1), (6.2) or (6.3) for all $t$, in which $p_{t+1}^{e}=p_{t+1}$ and $x_{t+1}^{e}=x^{*}$. By reproducing the argument that we used to prove Proposition 2.3 , one obtains

PROPOSITION 6.1. Assume $(1 . a),(1 . c)$. Let $x^{*}=1+r^{*}$ and $M_{0}$ be fixed. Consider a sequence $\left(p_{t}\right)$ such that $p_{t+k}=\left(x^{*}\right)^{k} p_{t}$ in which $k$ is the (primitive) period of the sequence. Then $\left(p_{t}\right)$ is a periodic equilibrium with perfect foresight corresponding to $r^{*}$ if and only if it satisfies for all $t$

$$
\begin{aligned}
& z_{1}\left(\theta_{t}\right)+z_{2}\left(\theta_{t-1}\right)=0 \\
& M_{t}=p_{t} z_{2}\left(\theta_{t-1}\right)
\end{aligned}
$$

in which $\theta_{t}=p_{t} x^{*} / p_{t+1}>\bar{\theta}$.

The system $(6.4),(6.5)$ is in fact identical to $(2.6),(2.7)$ which characterized periodic equilibria with perfect foresight in the no interest payment case, the only difference being that money grows now at

The assertion that the "average" growth rate of prices is $r^{*}$ along the cycle is justified by the following consideration. One has indeed

$$
\sum_{j=1}^{k} \log \left(p_{t+j} / p_{t+j-1}\right)=k \log \left(1+r^{\star}\right)
$$


the ratẽ $r^{\star}$ - and prices also, at least on average - instead of being constant. Real equilibrium magnitudes are the same in the two cases. The real part of the set of periodic equilibria with perfect foresight is thus not affected by a change of the level of the money stock $M_{0}$ or of the target growth rate $r^{*}$ of the money supply. Put it in another way, any given ousiness cycle appears to be "compatible" with any (average) rate of inflation ${ }^{1}$.

This analysis has an important qualitative implication. The foregoing characterization was achieved by making no reference to the specific features of the Government's policy beyond the fact that it aimed at pegging the long run rate of growth of the money supply at the predetermined level $r^{*}$. Thus the consequence of Proposition 6.1 is that monetary policy through nominal interest payments cannot create new cycles (in real terms) by comparison to those which already exist under laisser faire. Of course, the Government may try to do so by pegging real variables in a way that is not compatible with laisser faire periodic equilibria. But the consequence will be that the economy will never be able to settle down to a long run (i.e. periodic) monetary equilibrium. Either its trajectory will be chaotic (aperiodic) or it will

It is clear that the long run equilibrium "trade off" between output (or real balances) and real interest rates is also unaffected by a change of the target growth rate $r^{*}$. It is indeed still described by $y_{t}=f\left(\theta_{t-1}\right)\left(\right.$ or $\left.\mu_{t}=z_{2}\left(\theta_{t-1}\right)\right)$ in which $f$ is defined as in (5.4). The reader will verify easily on the other hand that the short run "trade offs" $y_{t}=F\left(\theta_{t-1}^{e}, e_{t}\right)$ and $\mu_{t}=e_{t} z_{2}\left(\theta_{t-1}^{e}\right)$ defined in (5.7) and (5.8) do not depend at all on the policy implemented by the monetary authority. 
collapse to the nonmonetary (no trade) equilibrium $\theta=\bar{\theta}$. The conclusion is clear. The only goal that the Government may pursue consistently - at least if it wishes to avoid chaos and to preserve the monetary character of the economy - is to design a policy that forces the economy to settle down at a particular long run laisser faire periodic equilibrium that is different from the ones that would have been naturally selected in the absence of any Government's intervention.

We shall show later on that there exists indeed a very simple countercyclical monetary policy that enables the monetary authority to force the economy back to the unique monetary steady state $\theta=1$, and to control at the same time the growth rate of the money supply. But we wish to show first that in order to have any real effect, the policy must be active, that is, it must try to influence some real variable in the system. More precisely, we wish to show that the monetary authority will be unable to have any real influence even on the dynamics of the economy during the adjustment process toward long run equilibria if it implements a passive policy, i.e. if it pegs permently the nominal interest rate at the predetermined level $r^{*}$ without attempting to control a real variable in the system - at least when traders believe that such a passive policy is indeed neutral. The consequence of this finding is clear. Proposition 6.1 says that with a passive policy of this sort, the set of corresponding periodic equilibria is the same (in real terms) as under laisser faire. What we just said implies that if consumers think that a passive policy is neutral, then such a passive policy is unable to affect the dynamic stability or unstability of long run periodic competitive equilibria. In this sense, a (passive) manipulation of the rate of growth of the maney supply through interest payments is superneutral. 
To see more precisely the point, let us assume that the economy has evolved under laisser faire until the date $t=0$, and that the Government steps in at that date and announces that it will pay the interest $r^{*}$ on money balances from $t=1$ onward. The corresponding evolution of the money stock is then $M_{t}=\left(1+r^{\star}\right)^{t} M$ for all $t \geqq 0$. Assume next that traders believe that this policy has no real effects even out of long run (periodic) equilibria. They think accordingly that the only consequence of making the transfer $x^{*}=1+r^{*}$ at date $t$, by comparison to the no transfer case $\left(x_{t}=0\right)$, is simply to multiply equilibrium prices at that date and at all future dates by the factor $x^{*}$. Or in other words that equilibrium prices are not affected by the transfers provided that they are properly discounted back to $t=0$. Formally, if we introduce the discount factors $\beta_{t}=1 /\left(1+r^{*}\right)^{t}$ for al1 $t \geqq 0$, the traders' expectations will be given by

$$
\beta_{t+1} p_{t+1}^{e}=\psi\left(\beta_{t} p_{t}, \ldots, \beta_{t-T} p_{t-T}\right)
$$

for all $t$ (with the additional convention that $\beta_{t}=1$ whenever $t<0$ ), in which $\psi$ is the expectation function that the traders would use in the absence of any Government's intervention, as in Section 2.

The dynamic evolution of the economy under these assumptions is then obtained by replacing $p_{t}^{e}$ by its expression and $x_{t+1}^{e}$ by $\left(1+r^{*}\right)$ in the temporary equilibrium equation (6.1). This yields

$$
z_{1}\left(\beta_{t} p_{t} / \psi\left(\beta_{t} p_{t}, \ldots, \beta_{t-T} p_{t-T}\right)\right)+\left(M / \beta_{t} p_{t}\right)=0
$$

We see therefore that the sequence $\left(p_{t}\right)$ is a sequence of temporary equilibrium prices when the interest rate is pegged at the level $r^{*}$ if and only if the sequence of discounted prices $\left(\beta_{t} p_{t}\right)$ is a sequence 
of equilibrium prices under laisser faire. Real equilibrium magnitudes are in fact the same along the two trajectories (think of the old trader whose equilibrium excess demand is $\left.M_{t} / p_{t}=M / \beta_{t} p_{t}\right)$, which establishes our claim.

It is intuitively clear that the same sort of superneutrality results holds, by applying the same reasoning, if the transfers $x_{t}=\left(1+r_{t}\right)$ may vary over time, but are predetermined at each date $t, i . e$. if $x_{t}$ depends on previously observed variables but not on current observables such as the current price $p_{t}$. The point is that if the transfer $x_{t}$ is not tied in some way to $p_{t}$, the monetary authority can have no hope to influence real interest rates. The formal details are left to the reader.

The discussion shows that if we wish to study whether the Government is able to influence real matters by means of interest payments, we must admit the possibility that the transfer $x_{t}$ is linked in some way to the current price $p_{t}$ - and to past prices - so as to influence real interest rates. And we may add that this is only fair. For we are playing an abstract and somewhat unrealistic game in which individual traders and market prices adjust infinitely fast and costlessly at each date, and we much allow the monetary authority to react as fast and on the basis of the same information as the private sector (the issue of diagnosing whether or not monetary authorities can in practice react as fast as the market being an important but entirely distinct matter).

We show now that there exists a very simple active countercyclical ("leaning against the wind") policy that enables the Government to force the economy back to the unique monetary stationary state $\theta=1$, 
and at the same time to peg the nominal interest rate. Let us assume that the economy has evolved under laisser faire before $t=0$ and that it has settled down for instance to a stable periodic equilibrium with period $k \geqq 2$. At the date $t=0$, the Government steps in and announces at that date that it will pay interest on money from $t=1$ onward according to the following policy. Its goal is to peg the nominal interest rate at the predetermined level $r^{*}$, as well as the real interest rate at $\theta=1$. This means that Government wants the evolution of the money supply and of equilibrium prices to be (see $(6.2))$

$$
M_{t}^{*}=\left(1+r^{*}\right)^{t} M \quad \text { and } \quad p_{t}^{*}=M_{t}^{*} / z_{2}(1)
$$

Of course, all this presupposes that $\bar{\theta}<1$ (assumption $(1 . d)$ ). One possible way to achieve this goal is to adopt the following simple feedback rule

$$
x_{t+1}=1+r_{t+1}=\left(p_{t+1} / p_{t}\right) g\left(p_{t} / p_{t}^{*}\right)
$$

for $t \geqq 0$. In other words, this rule ties the real interest rate between dates $t$ and $t+1$ (or the value of $\theta_{t}=\frac{p_{t} x_{t+1}}{p_{t+1}}$ ) to the deviation of the price $p_{t}$ from the target price $p_{t}^{*}$ for the date $t$. Note that $\theta_{t}-1$ may be interpreted equivalently as the real rate of growth of the money supply between dates $t$ and $t+1$, since $\theta_{t}=\left(M_{t+1} / p_{t+1}\right) /\left(M_{t} / p_{t}\right)$. of course, one should have $g(1)=1$ for the rule to be consistent with the objectives of the Government. The policy is thus characterized by $r^{*}$ and the function $g$, which maps $(0,+\infty)$ into itself, satisfying $(6.6)$, (6.7) and $g(1)=1$. We shall assume for simplicity that $g$ is differentiable. The policy will be said to be countercyclical if a large 
deviation $\mathrm{p}_{\mathrm{t}} / \mathrm{p}_{\mathrm{t}}^{*}$ is associated to a low real growth rate of the money sypply, that is if $g^{\prime}<0$.

Suppose now that the Government's policy $\left(r^{*}, g\right)$ is public knowledge at a11 dates $t \geqq 0$. This information will therefore influence the way in which the traders' anticipations are formed. In fact, one must have

$$
p_{t+1}^{e} / x_{t+1}^{e}=p_{t} / g_{t}\left(p_{t} / p_{t}^{*}\right)
$$

at all dates. The temporary equilibrium equation (6.1) or (6.2) becomes then, for al1 $t \geqq 0$

$$
z_{1}\left(g\left(p_{t} / p_{t}^{*}\right)\right)+\left(M_{t} / p_{t}\right)=0
$$

Therefore, under the policy $\left(r^{*}, g\right)$, the evolution of temporary equilibrium prices for $t=0,1, \ldots$, is governed by $(6.8)$, in which of course

$$
M_{t+1}=x_{t+1} M_{t} \text { for all } t \geqq 0 \text {, and } M_{0}=M
$$

The next result states that the unique solution of that dynamical system is $p_{t}=p_{t}^{*}$ and $M_{t}=M_{t}^{*}$ for all $t \geqq 0$, i.e. that this stabilization policy is successful, provided that $z_{1}^{\prime}(1)>0$, and that the policy is only "moderately" countercyclical, in the sense that the range of variation of $\mathrm{g}$ is smalt ${ }^{1}$.

One may note that under assumption (1.e), the condition $z_{1}^{\prime}(1)>0$ is necessary for the existence of a cycle of period $k \geqq 2$. See Lemma 4.2 . 
PROPOSITION 6.2. Assume $(1 . a),(1 . c),(1 . d)$, and that the Government adopts the (publicly known) policy $\left(r^{*}, g\right)$ from $t=0$ onward, in which $r^{*}$ and $g$ satisfy $(6.6),(6.7)$ and $g(1)=1$. Assume moreover that the function $g$ is differentiable, that $g^{\prime}(v)<0$ and that there exists $\varepsilon>0$ such that $|g(v)-1| \leqq \varepsilon$ for all $v>0$.

Then if $z_{1}^{\prime}(1)>0$ and if $\varepsilon$ is small enough, the sequence of temporary equilibrium prices $\left(p_{t}\right)$ and of equilibrium money stocks $M_{t}$ corresponding to this policy is unique and is given by

$$
M_{t}=M_{t}^{*}=\left(1+r^{*}\right)^{t} M \text { and } p_{t}=p_{t}^{*}=M_{t}^{*} / z_{2}(1)
$$

for all $t \geqq 0$. Moreover the temporary equilibrium is stable at each date in any walrasian tatonnement process in which prices respond positively to excess demand.

As the preceding statement makes clear, what matters really is the "countercyclical" part of the policy that is described by the function $\mathrm{g}$. On the other hand, an arbitrary variation of the target growth rate $r^{*}$ of the money supply, the function $g$ being fixed, is superneutral : it induces only an offsetting variation of the rate of inflation, so that the stabilized real interest rate remains equal to 0 . We see here an instance of a phenomenon that seems rather general, i.e. the fact that a permanent change of the trend value of the growth rate of the money supply (here $r^{*}$ ) may have an impact that is very different from the consequences of the "transitory" (countercyclical) part of the policy (here the map g). As a final remark, it is worthwhile to note that a successful stabilization policy like the above has not only an impact on the variances of real equilibrium magnitudes such as output 
or real balances, but also influences generally their means li.e. the averages over a cycle of the values that they takel since the model is highly nonlinear ${ }^{1}$.

We end this Section by the proof of Proposition 6.2. It is very simple and proceeds by induction on $t$. Consider first what happens at the initial date $t=0$. The good market equilibrium equation (6.8) for that period reads

$$
z_{1}\left(g\left(p_{0} / p_{0}^{*}\right)\right)+\left(M / p_{0}\right)=0
$$

Since $z_{1}(1)+z_{2}(1)=0$, this equation admits obviously the solution $p_{0}=p_{0}^{*}=M / z_{2}(1)$. In order to show that this solution is unique and stable in an arbitrary Walrasian tatonnement, it suffices to show that the left hand side of the equation is decreasing. Since $z_{1}^{\prime}(1)>0$, then by continuity, there exists $\varepsilon>0$ such that $z_{1}^{\prime}(\theta)>0$ if $|\theta-1| \leqq \varepsilon$. Thus if $|g(v)-1| \leqq \varepsilon$ and if $g^{\prime}(v)<0$ for all $v>0$, then the derivative of $z_{1}(g(v))$ is negative for all v, which proves the claim. We remark finally that if the equilibrium solution at $t=0$ is $p_{0}=p_{0}^{*}$, then we know from (6.7) and (6.9) that the transfer $x_{1}$ will vary with the price $p_{1}$ prevailing at $t=1$ so as to keep the real money stock $M_{1} / p_{1}$ equal to $z_{2}(1)$.

\footnotetext{
An interesting issue that has not been investigated in the present work is to find conditions such that reducing for instance the variance of equilibrium output raises at the same time its mean value in contrast for instance to Sargent and Wallace (1975). This is a topic for further research.
} 
We proceed now by induction and suppose that (6.8) admits the unique solution $p_{j}=p_{j}^{*}$ for $j=0, \ldots, t-1$. By the same argument that we just employed, we know then that the transfer $x_{t}$ at the date $t$ will be adapted by the monetary authority to the price $\mathrm{p}_{\mathrm{t}}$ prevailing at that date so as to keep the real money stock $M_{t} / p_{t}$ equal to $z_{2}(1)$. The good market equilibrium equation $(6.8)$ for date $t$ can then be written as

$$
z_{1}\left(g\left(p_{t} / p_{t}^{*}\right)\right)+z_{2}(1)=0
$$

Again, $p_{t}=p_{t}^{*}$ is a solution. To show that it is unique and stable in an arbitrary Walrasian tatonnement, it suffices here again to show that the left hand side of the equation is a decreasing function of $\mathrm{p}_{t}$. By the same reasoning as for $t=0$, this is true when $z_{1}^{\prime}(1)>0$, $|g(v)-1| \leqq \varepsilon$ and $g^{\prime}(v)<0$ for all $v>0$, in which the parameter $\varepsilon$ is the same as for $t=0$. This completes the proof of the Proposition ${ }^{l}$.

The structure of the foregoing argument shows that $p_{t}=p_{t}^{*}$ and $M_{t}=M_{t}^{*}$ is always a solution of (6.8) without any restriction on the policy $g$ other than $g(1)=1$. The restrictions $z_{1}^{\prime}(1)>0, g^{\prime}(v)<0$ $\left|g^{\prime}(v)-1\right| \leqq \varepsilon$ with $\varepsilon$ small enough are there to guarantee the unicity of this solution and its stability at each date under a Walrasian tatonnement. In the absence of such restrictions, two phenomena could occur. First, there might be another solution that would correspond to a cycle of the economy under laisser faire, with a period $k \geqq 2$ (this could occur if the range of variation of $g$ is "large"). Second even in the case in which $\theta_{t}=1$ would be the unique solution, the level of money prices might be indeterminate (think of the case in which $g(v)=1$ for a $11 \mathrm{v}$ ). 
Remarks.

1. It should be emphasized that the various "superneutrality" results that were reached in this Section, e.g. the invariance in real terms of the set of periodic equilibria, or of the long run "trade off" between equilibrium output and real interest rates with respect to a change of the target growth rate $r^{*}$, or the superneutrality of "predetermined" interest payments no longer hold if money is injected in the economy by other means such as lump sum transfers or Government's purchases of goods. The basic reason is simple : a change of the rate of growth of the money supply through lump sum transfers or Government's purchases is bound to alter, for some time or permanently, the real rate of interest on money through a variation of the rate of inflation, whether or not the change of the growth rate of the money supply is temporary or permanent, predetermined

or not, anticipated or not. Such policies can therefore create new cycles as well as stabilize the economy. All this should be the subject of further research.

The distinction between the effects of a variation of the rate of growth of the money supply through nominal interest payments and through lump sum transfers has a long history in economics. It is in particular at the heart of Friedman's theory of the Optimum Quantity of money (1969, Ch. 1). See also Grandmont and Younès (1973) and the discussion in Grandmont (1983a,Ch. 1, Section 7). This distinction has been used again recently in the perspective of stabilization policy in an overlapping generation model by Azariadis and Balasko (1983), Stiglitz (1983). 
The effects of a variation of the money supply through the Government's purchases has been studied recently in a (log-linear) version of the overlapping generation model by S. Fisher (1979).

2. The simple result that monetary policy is able to "lock" immediately the economy into the stationary state seems to be due to the specificity of the model. If traders 1 ived for more than two periods, for instance, matters would be less simple, since when the Government steps in at $t=0$, the initial distribution of money holdings among traders will be typically different from the one that characterizes a stationary state, and this may have lasting effects on the trajectory of the economy no matter which policy is implemented by the monetary authority. 
7. CONCLUSTON.

The model that we have analyzed in this paper is obviously too rudimentary to enable us to draw very general conclusions. Yet, the study of this particular example is suggestive and shows that it is possible to construct plausible models in which endogenous and significant fluctuations obtain that are not caused by exogenous shocks nor by policy. Furthermore a properly designed countercyclical policy can stabilize these autonomous business cycles. Such results are manifestly at variance with the conclusions that yield usually currently fashionable Classical "loglinear" macroeconomic models. It remains to be seen whether or not these results carry over to more general and more realistic structures. They suggest at any rate that economic theorists should perhaps look more closely at the sort of mechanisms that may be responsible for significant nonlinearities in the economic system if they wish to have a proper foundation upon which to build a sound business cycle theory.

Among the issues that appear worthwhile to investigate, one may mention the following

1. One importarit feature of the model that has been studied is the fact - that seems likely to obtain as well within more sophisticated frameworks - that cycles of different periods typically coexist. This raises, as we have seen, the question of the stability of a given cycle, and our analysis showed that any answer to that question depends crucially on the properties of the traders' learning processes, We have in fact touched upon this problem only incidentally in Section 3 , when establishing a relationship between stability with learning and stability under the perfect foresight backward dynamics. A deeper examination 
of the stability of a cycle, for instance by adapting and simplifying the methods of Fuchs and Laroque (1976), would be useful,

2. Although we have deliberately ignored exogenous shocks in the present analysis in order to focus attention on autonomous business cycles, it is clear that (random or deterministic) shocks that result from the economy's environment and/or from the Government's policy, as well as incomplete and asymmetric information, play some role in the generation of modern economic fluctuations - although their importance may not be as great as some "New Classical macroeconomists" would like to believe. The integration of such exogenous shocks, for instance along the lines of Lucas's model (1972), would be interesting as it would allow making precise how they interact with the endogenous mechanisms in the generation of economic fluctuations.

3. A related issue concerns the fact already mentioned in Section 6 that deterministic economic policies through lump sum money transfers, income taxes, Government purchases of goods, open market operations are able to generate economic fluctuations as well as to stabilize them. It would be important to proceed to a systematic study of the existence and the properties of the cycles that may emerge in the present model when the Government is implementing a given policy through these alternative channels.

4. As we have said, the central mechanism through which endogenous business cycles emerge in the present model is the conflict between the intertemporal substitution effect and the wealth effect that are associated to a variation of the real rate of interest. 
An interesting study would be to extend the analys is to a more general framework, in which for instance traders would live an arbitrary (finite or infinite) number of periods. Another topic of particular interest is to discover other mechanisms - for instance associated to particular features of the production technology - that yields nonlinearities sufficient to generate endogenous business cycles.

5. The whole analysis has been carried out under the ad hoc assumption that market clear at every moment in the Walrasian sense. An important topic for future research would be to relax this somewhat unrealistic assumption, for instance by introducing imperfect competition in the model, and to look for the kind of mechanisms that may give rise to endogenous economic fluctuations. In a Walrasian framework, one may get economic fluctuations only through a variation of relative prices, that is (in a macroeconomic context involving only one good as in the present mode1) only through a variation of the real interest rate. By contrast, once imperfect competition is taken into account, quantity adjustments, and thus something like multiplier or accelerator effects, have to play a role. One may then hope to get a better foundation upon which to build a sound Keynesian, or more precisely nonWalrasian, business cycle theory. 
REFERENCES.

ARROW, K.J. (1970), Essays in the Theory of Risk Bearing, London, North Holland.

AZARIADIS, C. (1981), "Self-Fulfilling Prophecies", Journal of Economic Theory $25,380-396$.

AZARIADIS, C. and Y. BALASKO (1983), "The Pigou Effect and the Phillips Correspondence", University of Pennsylvania, Mimeo.

AZARIADIS, C. and R. GUESNERIE (1983), "The persistence of Self-Fulfilling Theories", forthcoming.

BARRO, R.J. (1981), "The Equilibrium Approach to Business Cycles", Ch. 2 in Money Expectations, and Business Cycles, Academic Press, New York.

BENHABIB, J. and R.H. DAY (1981), "Rationa? Choice and Erratic Behaviour", Review of Economic Studies 48, 459-472.

BENHABIB, J. and R.H. DAY (1982), "A Characterisation of Erratic Dynamics in the Overlapping Generations Model", Journal of Economic Dynamics and Control 4, 37-55.

BRAY, M. (1982), "Learning, Estimation and the Stability of Rational Expectations", Journal of Economic Theory 26, 318-339.

CASS, D. (1980), "Money in Consumption-Loan Type Models : An Addendum", in J.H. KAREKEN and N. WALLACE (Eds), Models of Monetary Economies, Federal Reserve Bank of Minneapolis.

CASS, D, and K. SHELL (1981), "Do Sunspots Matters?" CARESS Working Paper 8009 R, University of Pennsylvania, 1981. Also appearing in French as "Les taches solaires ont-elles de l'importance ?". Cahiers du Séminaire d'Econométrie 24 (1982), 93-127. 
CASS, D. and K. SHELL (1983), "Do Sunspots Matter ?" Journal of Political Economy 91 , forthcoming.

CHAMPSAUR, P. (1983), "On the Stability of Rational Expectations Equilibria", CORE Discussion Paper 8324, Université Catholique de Louvain.

COLLET, P. and J.-P. ECKMANN (1980), Iterated Maps on the Interval as Dynamical Systems, Birkhaüser, Boston.

DANA, R.A. and P. MALGRANGE (1981), "The Dynamics of a Discrete Version of a Growth Cycle Mode1", CEPREMAP Working Paper, forthcoming in Analysing the Structure of Econometric Models, J.P. ANCOT (Ed.), M. Nijhoff, Amsterdam.

DAY, R.H. (1982), "Irregular Growth Cycles", American Economic Review 72, 406-414.

DAY, R.H. (1983), "The Emergence of Chaos from Classical Economic Growth", Quarterly Journal of Economics 98, 201-13.

DIAMOND, P. and D. FUDENBERG (1983), "An Example of Rational Expectations Business Cycles in Search Equilibrium", M.I.T., Mimeo.

FISHER, S. (1979), "Anticipations and the Nonneutrality of Money", Journal of Political Economy 87, 225-52.

FRIEDMAN, B. (1979), "Optimal Expectations and the Extreme Information Assumptions of 'Rational Expectations' Macromode1s", Journal of Monetary Economics 5, 23-41.

FRIEDMAN, M. (1969), The Optimum Quantity of Money and Other Essays, Chicago, Aldine.

FRIEND, I. and J. HASBROUCK (1981), "Effects of Inflation on the Profitability and Valuation of U.S. Corporations", Working Paper 3-81, Rodney White Center for Financial Research, University of Pennsylvania, Philadelphia. 
FUCHS, G. (1976), "Asymptotic Stability of Stationary Temporary Equilibria and Changes in Expectations", Journal of Economic Theory 12, 201-216.

FUCHS, G. (1977a), "Dynamic Role and Evolution of Expectations", in Systèmes dynamiques et modèles économiques, Proceedings of a C.N.R.S. International Meeting, Vol. 259, 183.

FUCHS, G. (1977b), "Formation of Expectations. A Model in Temporary General Equilibrium Theory", Journal of Mathematical Economics 4, 167-188.

FUCHS, G. (1979a), "Dynamics of Expectations in Temporary General Equilibrium Theory", Journal of Mathematical Economics 6, 229-252.

FUCHS, G. (1979b), "Are Error Learning Behaviours Stabilizing ?", Journal of Economic Theory 3 , 300-317.

FUCHS, G. and G. LAROQUE (1976), "Dynamics of Temporary Equilibria and Expectations", Econometrica 44, 1157-1178.

GALE, D. (1973), "Pure Exchange Equilibrium of Dynamic Economic Models", Journal of Economic Theory 6, 12-36.

GRANDMONT, J.M. (1983a), Money and Value, Econometric Society Series, Cambridge University Press.

GRANDMONT, J.M. (1983b), "Periodic and Aperiodic Behaviour in Discrete Onedimensional Dynamical Systems", CEPREMAP D.P. N ${ }^{\circ} 8317$. Also available as a Technical Report of IMSSS, Economics, Stanford University and a Technical Report of EHEC, University of Lausanne.

GRANDMONT, J.M. and W. HILDENBRAND (1974), "Stochastic Processes of Temporary Equilibria", Journal of Mathematical Economics 1, 247-277.

GRANDMONT, J.M. and G. LAROQUE (1973), "Money in the Pure Consumption Loan Model", Journal of Economic Theory 6 , 382-395.

GRANDMONT, J.M. and Y. YOUNES (1973), "On the Efficiency of a Monetary Equilibrium", Review of Economic Studies 40, 149-165.

HAHN, F.H. (1982), Money and Inflation, Basil Blackwel1, Oxford.

JENSEN, R.U. and R. URBAN (1982), "Chaotic Price Behaviour in a Nonlinear Cobweb Model", Yale University, mimeo. 
KYDLAND, F.E. and E,C, PRESCOTT (1982), "Time to Build and Aggregate Fluctuations", Econometrica 50, 1345-1370.

LI, T, and J.A. YORKE (1975), "Period Three Implies Chaos", American Mathematical Monthly 82, 985-992.

LUCAS, R.E. Jr. (1972), "Expectations and the Neutrality of Money", Journal of Economic Theory 4, 103-124.

LUCAS, R.E., Jr. (1975), "An Equilibrium Model of the Business Cycle", Journal of Political Economy 83, 1113-44.

LUCAS, R.E., Jr. (1977), "Understanding Business Cycles", Journal of Monetary Economics 3 (Supplement), 7-30.

LUCAS, R.E., Jr. (1980), "Methods and Problems in Business Cycie Theory", Journal of Money, Credit and Banking 12, 696-715.

LUCAS, R.E., Jr. (1981), "Tobin and Monetarism : A Review Article", Journal of Economic Literature 19, 558-567.

MCCALLUM, B.T. (1980), "Rational Expectations and Macroeconomic Stabilization Policy", Journal of Money, Credit and Banking 12, 716-746.

MAY, R.B. (1976), "Simple Mathematical Models with Very Complicated Dynamics", Nature 261, 459-467.

RAND, D. (1978), "Exotic Phenomena in Games and Duopoly Models", Journal of Mathematical Economics 5, 173-184.

SARGENT, T.J. and N. WALLACE (1975), "Rational Expectations, the Optimal Monetary Instrument, and the Optimal Money Supply Rule", Journal of Political Economy 83, 241-254.

SHELL, K. (1977), "Monnaie et allocation intertemporelle", CNRS Séminaire d'Econométrie Roy-Mal invaud, Paris, mimeo. 
SINGER, D, (1978), "Stable Orbits and Bifurcations of Maps of the Interval", SIAM Journal of Applied Mathematics 35, 260.

STIGLITZ, J,E, (1983), "On the Relevance or Irrelevance of Public Financial Policy : Indexation, Price Rigidities and Optimal Monetary Policy", NBER Working Paper 1106.

TILLMANN, G. (1983), "Stability in a Simple Pure Consumption Loan Mode1", Journal of Economic Theory 30, 315-329.

TOBIN, J. (1980), Asset Accumulation and Economic Activity, Yrjö Jahnsson Lectures, Basil Blackwel1, Oxford.

WILKINSON, J.H. (1965), The Algebraic Eigenvalue Problem, Clarendon Press, Oxford. 


\section{A P PENDIX}

A. STABILITY AND LEARNING.

The purpose of this Section is to prove Proposition 3.1 . We assume therefore $(1 . a),(1 . c),(2.6),(2 . g)$ and $(3 . h)$, and consider a periodic equilibrium $\left(p_{1}^{*}, \ldots, p_{k}^{*}\right)$ and the associated cycle $\left(\theta_{k}^{*}, \ldots, \theta_{1}^{*}\right)$ of the map $\varphi$. We wish to show that if $\left(\theta_{k}^{*}, \ldots, \theta_{1}^{*}\right)$ is $\varphi$-stable, then $\left(p_{1}^{*}, \ldots, p_{k}^{*}\right)$ is stable in the sense of (3.1).

As a matter of fact, it wi11 be more convenient not to work directly with the backward perfect foresight dynamics on equilibrium real interest rates described by

$$
\theta_{t-1}=\varphi\left(\theta_{t}\right)=z_{2}^{-1}\left(z_{2}\left(\theta_{t}\right) / \theta_{t}\right)
$$

but rather to use the backward perfect foresight dynamics on equilibrium prices that is deduced from (A.1) through the transformation (see (2.7))

$$
p_{t}=M / z_{2}\left(\theta_{t-1}\right)
$$

This yields

$$
\left.p_{t}=M \theta_{t} / z_{2}\left(\theta_{t}\right)=p_{t+1} z_{2}^{-1}\left(M / p_{t+1}\right)\right)
$$

Note that $(A .2)$ defines a differentiably decreasing function $p_{t}=\zeta\left(\theta_{t-1}\right)$ that maps $(\bar{\theta},+\infty)$ onto $(0,+\infty)$. On the other hand, $(A, 3)$ defines a function $p_{t}=\Phi\left(p_{t+1}\right)$ that maps the interval $(0,+\infty)$ into itself, and one has $\Phi=\zeta 0 \varphi \circ \zeta^{-1}$. Then it is not difficult to see that 
$\left(p_{k}^{*}, \ldots, p_{1}^{*}\right)$ is a cycle of $\Phi$, and that the periodic orbit $\left(\theta_{k}^{*}, \ldots, \theta_{1}^{*}\right)$ is $\varphi$-stable if and only if

$$
\left|D \Phi^{k}\left(p_{1}^{*}\right)\right|=\left|\Phi^{\prime}\left(p_{1}^{*}\right)\right| \ldots\left|\Phi^{\prime}\left(p_{k}^{*}\right)\right|<1
$$

What we have to show accordingly is that if this condition is satisfied then all the eigenvalues of the Jacobian matrix $D \tilde{W}^{k}\left(q_{1}^{*}\right)$ (or equivalently of $D \tilde{W}^{k}\left(q_{j}^{*}\right)$ ) have a modulus less than 1 , in which $\left(q_{1}^{*}, \ldots, q_{k}^{*}\right)$ is the associated periodic orbit of $\tilde{W}$ as in Lemma 2.2 , i.e.

$$
q_{i}^{*}=\left(p_{i}^{*}, \ldots, p_{1}^{*}, p_{k}^{*}, \ldots, p_{1}^{*}, \ldots\right)
$$

for $i=1, \ldots, k$.

The advantage of working with the map $\Phi$ instead of the map $\varphi$ is most clearly seen if one remarks that (A.3) may be rewritten as

$$
p_{t+1} z_{2}\left(p_{t} / p_{t+1}\right)=M
$$

This is nothing else, of course, than the temporary equilibrium equation (2.3) in which the expected price $\psi\left(p_{t}, p_{t-1}, \ldots, p_{t-T}\right)$ has been replaced by its "true" value $p_{t+1}$. Since (2.3) defines implicitly the temporary Walrasian equilibrium function, we have

$$
W\left(p_{t-1}, \ldots, p_{t-T}\right) \equiv \Phi\left[\psi\left(W\left(p_{t-1}, \ldots, p_{t-T}\right), p_{t-1}, \ldots, p_{t-T}\right)\right]
$$

As an incidental remark, we note that we may assume without any loss of generality, for the purpose of the present proof, that $T$ is a multiple of $k$, that is $T=m k$ for some integer $m \geqq 1$. Indeed it is always possible to take into account an arbitrary number of additional past prices as fictitious arguments of the expectation function $\psi$. 
Let $W_{j}^{\prime}\left(q_{j}^{*}\right)$ be the partial derivative of $W$ with respect to $p_{t-j}$ for $j=1, \ldots, T$, evaluated at $\left(p_{t-1}, \ldots, p_{t-T}\right)=q_{j}^{\star}$. By differentiating $(A .4)$ and by using assumptions (2.g) and $(3 . h)$ (or equivalentiy (3.4)), we get

(A.5) For every $i=1, \ldots, k$, one has $w_{j}^{\prime}\left(q_{j}^{*}\right)=0$ for al1 $j=1, \ldots$, $T$ (=mk) , except for those of the form $j=n k-1$, for which one has $w_{j}^{\prime}\left(q_{i}^{*}\right)=\Phi^{\prime}\left(p_{i+2}^{*}\right) \psi_{j}^{\prime}\left(p_{i+1}^{*}, q_{j}^{*}\right)$ (here the integer $n$ runs from 2 to $m+1$ when $k=1$, and from 1 to $m$ when $k \geqq 2$ ). Accordingly, for all such $n, W_{n k-1}^{\prime}\left(q_{j}^{*}\right)$ has the same sign as $\Phi^{\prime}\left(p_{j+2}^{*}\right)$, and one has $\sum_{n} w_{n k-1}^{\prime}\left(q_{j}^{*}\right)=\Phi^{\prime}\left(p_{i+2}^{*}\right)$.

Our next task is to evaluate the Jacobian matrix $D \tilde{W}^{k}\left(q_{1}^{*}\right)$. We first remark that in view of (2.5), one has for every $q=\left(q_{1}, \ldots, q_{T}\right)$ in the interior of $\mathbb{R}_{+}^{\top}$

$$
\widetilde{W}(q)=\left(w(q), q_{1}, \ldots, q_{T-1}\right)
$$

Thus we get by differentiation of (A.6)

We use the convention $p_{k+h}^{*}=p_{h}^{*}$ and the notation $\psi_{j}\left(p_{i+1}^{*}, q_{j}^{*}\right)$ stands for $\psi_{j}^{\prime}\left(p_{i+1}^{*}, p_{i}^{*}, \ldots, p_{1}^{*}, p_{k}^{*}, \ldots\right)$. 


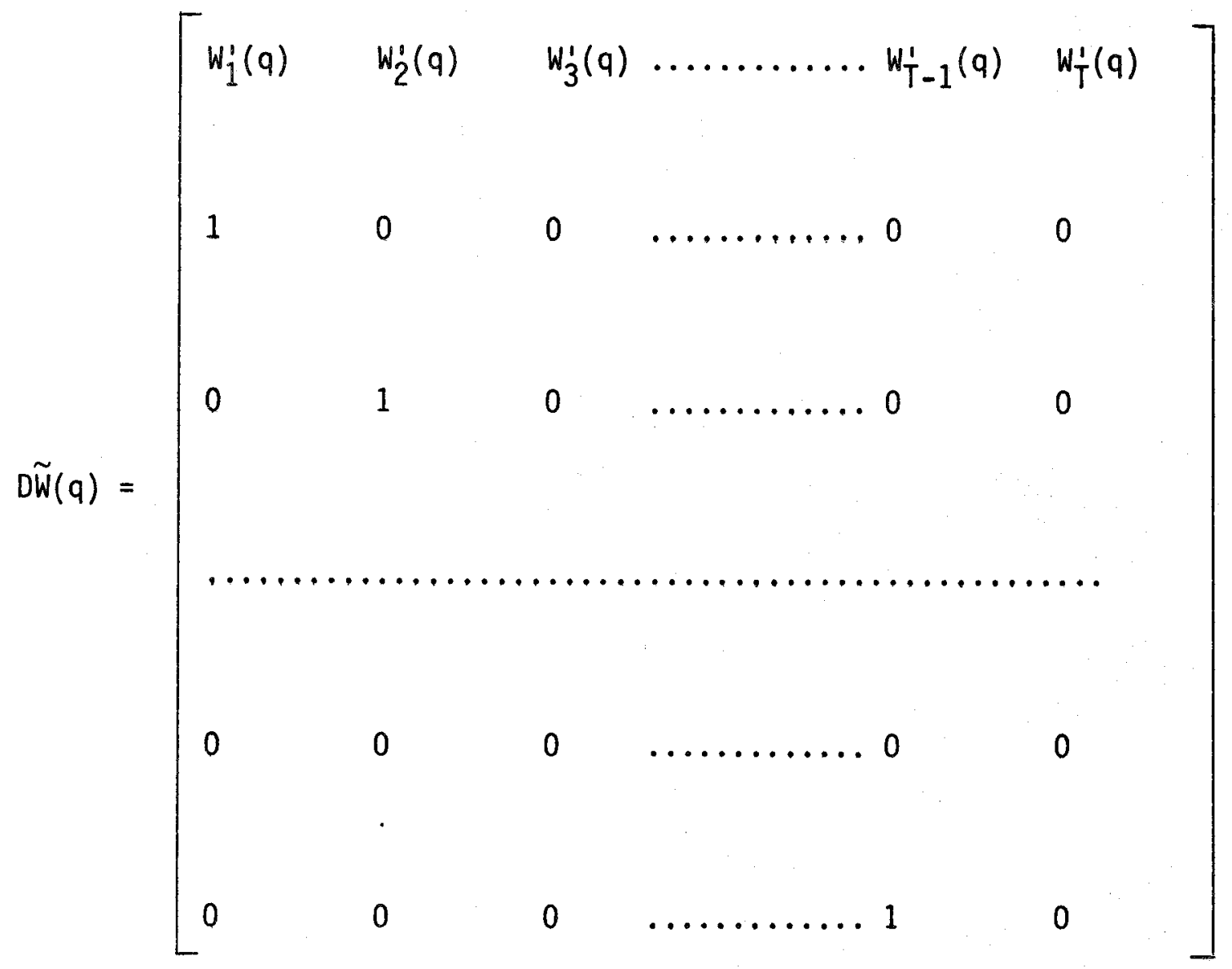

If $k=1$ (hence $T=m$ ), we are done since it suffices to make q equal to the stationary vector $q^{*}$ in the above matrix. When $k \geqq 2$, one could compute $D \tilde{W}^{k}\left(q_{1}^{*}\right)$ with the help of the above matrix by using the fact that $D \tilde{W}^{k}\left(q_{1}^{*}\right)=D \tilde{W}\left(q_{k}^{*}\right) \ldots D \tilde{W}\left(q_{1}^{*}\right)$ - see $(3.1)$ - and by using the restrictions stated in $(A .5)$. It turns out that it is more convenient to proceed directly. To this effect, we may introduce recursively the "iterates" of $W$ by $W^{1}=W$ and for $h \geqq 2$

$$
w^{h}(q)=w\left(w^{h-1}(q), \ldots, w(q), q_{1}, \ldots, q_{T-h+1}\right)
$$

That is, if we start with $q=\left(p_{t-1}, \ldots, p_{t-T}\right)$, then $w^{h}(q)$ is the temporary equilibrium price at date $t+h-1$, for all $h \geqq 1$. With this notation, it is clear that 


$$
\tilde{w}^{h}(q)=\left[W^{h}(q), \ldots, w(q), q_{1}, \ldots, q_{T-h}\right]
$$

In particular $w^{h}(q)=w\left(\tilde{W}^{h-1}(q)\right)$.

Let $a_{i j}$ be the element of $D \tilde{W}^{k}\left(q_{1}^{*}\right)$ that lies in the $i$ th row and the $j$ th column for $i, j=1, \ldots, T$. Of course $a_{i j}$ is the partial derivative of the $i$ th component of $\tilde{W}^{k}(q)$ with respect to $q_{j}$, evaluated at the point $q_{1}^{*}$ of the periodic orbit $\left(q_{1}^{*}, \ldots, q_{k}^{*}\right)$. Then partial differentiation of (A.8) with $h=k$, shows immediately that if $i \geqq k+1$, then $a_{i j}=1$ if $j=i-k$ and $a_{i j}=0$ if $j \neq i-k$. For $1 \leqq i \leq k, a_{i j}$ is the partial derivative of $w^{k-i+1}(q)$ with respect to $q_{j}$, evaluated at $q_{1}^{*}$, i,e. $\frac{\partial w^{k-i+1}}{\partial q_{j}}\left(q_{1}^{*}\right)$. Differentiating $(A, 7)$ with $h=k-i+1$ yields for all $i=1, \ldots, k$ and $j=1, \ldots, T$

$$
a_{i j}=\left[\sum_{\ell=1}^{k-i} W_{l}^{\prime}\left(q_{k-i+1}^{*}\right){\frac{\partial W^{k-i+1-\ell}}{\partial q_{j}}}^{*}\left(q_{1}^{*}\right)\right]+W_{k-i+j}^{\prime}\left(q_{k-i+1}^{*}\right)
$$

whenever $j \leqq T-k+i$. If on the contrary, $j>T-k+i$, then

$$
a_{i j}=\left[\sum_{\ell=1}^{k-i} w_{\ell}^{\prime}\left(q_{k-i+1}^{*}\right){\frac{\partial W^{k-i+1-\ell}}{\partial q_{j}}}^{*}\left(q_{1}^{*}\right)\right]
$$

Now if $2 \leqq i \leqq k$, then $k-i \leqq k-2$, and thus in view of (A.5), $W_{\ell}^{\prime}\left(q_{k-i+1}^{*}\right)=0$ for all $\ell \leqq k-i$ : all the terms of the sum between the brackets in (A.9) vanish. In that case, $a_{i j}=0$ for all $j=1, \ldots, T$, except for those $j$ that satisfy $k-i+j=n k-1$, or equivalently $j=(n k-1)-(k-i)$ for $n=1, \ldots, m$ (we recall that $T=m k)$. For those $j$, one has $a_{i j}=w_{n k-1}^{\prime}\left(a_{k-i+1}^{*}\right)$. 
Finally if we specialize (A.9) to the case in which $i=1<k$, then in view of $(A .5)$, we get that

$$
a_{1 j}=w_{k-1}^{\prime}\left(q_{k}^{*}\right) w_{j}^{\prime}\left(q_{1}^{*}\right)+w_{k+j-1}^{\prime}\left(q_{k}^{*}\right)
$$

if $j \leqq T-k+1$, and $a_{1 j}=W_{k-1}^{\prime}\left(q_{k}^{*}\right) W_{j}^{\prime}\left(q_{1}^{*}\right)$ otherwise. Using (A.5) again yields that $a_{1 j}=0$ for all $j$, except for those of the form $j=n k-1$ with $n=1, \ldots, m$, in which case $a_{1 j}=w_{k-1}^{\prime}\left(q_{k}^{*}\right) w_{n k-1}\left(q_{1}^{*}\right)$, and for those of the form $j=n k$ with $n=1, \ldots, m-1$, in which case $a_{1 j}=w_{(n+1) k-1}^{\prime}\left(q_{k}^{*}\right)$.

To summarize our findings, we see that we may partition, for every $k \geqq 1$, the Jacobian matrix $D \tilde{W}^{k}\left(q_{1}^{*}\right)$ into $m^{2}$ submatrices of dimensions $(k, k)$ as follows

$$
D W^{k}\left(q_{1}^{*}\right)=\left[\begin{array}{cccccccc}
A_{1} & A_{2} & A_{3} & \ldots \ldots & A_{m-2} & A_{m-1} & A_{m} \\
I & 0 & 0 & \ldots \ldots & 0 & 0 & 0 \\
0 & I & 0 & \ldots \ldots & 0 & 0 & 0 \\
\ldots & \ldots & \ldots & \ldots & \ldots & \ldots & \ldots & \ldots \\
0 & 0 & 0 & \ldots \ldots & 0 & 0 & 0 \\
0 & 0 & 0 & \ldots \ldots & I & 0 & 0 \\
0 & 0 & 0 & \ldots \ldots & 0 & 1 & 0
\end{array}\right]
$$


in which $I$ is the $(k, k)$ unit matrix, 0 is a $(k, k)$ matrix of which the elements are all 0 , and for each $n=1, \ldots, m$, the matrix $A_{n}$ is given by

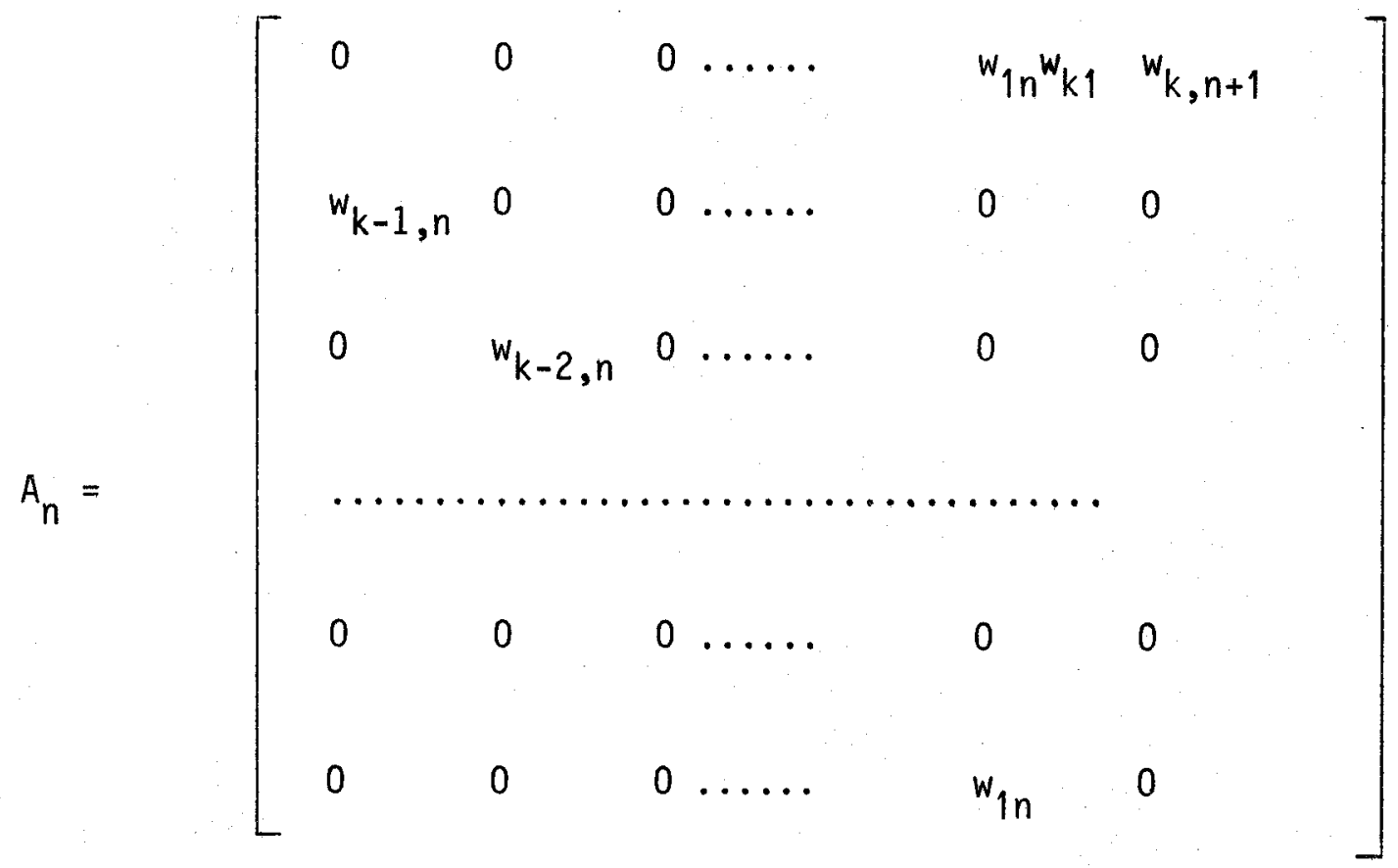

with $w_{i n}=w^{\prime}{ }_{n k-1}\left(q_{j}^{*}\right)$ for $i=1, \ldots, k$ and $n=1, \ldots, m$, and with the additional convention that $w_{k, m+1}=w^{\prime}(m+1) k-1\left(q_{k}^{*}\right)$ vanishes when $k \geqq 2$. Indeed, this translates exactly the results we obtained for the case $k \geqq 2$. As for the case $k=1$, each matrix $A_{n}$ reduces then to its top right element $w_{1, n+1}$, which is equal to $w_{n}^{\prime}\left(q^{*}\right)$ with $q^{*}$ being the fixed point of $\tilde{W}$ under consideration.

Note that with this notation, (A.5) means that given $i=1, \ldots, k$, al1 $w_{i n}$ have the same sign and $\sum_{n} w_{i n}=\Phi^{\prime}\left(p_{i+2}^{*}\right)$ (here again $n$ runs from 1 to $m$ when $k \geqq 2$ and from 2 to $m+1$ when $k=1$ ). 
Our purpose, we recal1, is to show that $\left|\Phi^{\prime}\left(p_{1}^{*}\right) \ldots \Phi^{\prime}\left(p_{k}^{*}\right)\right|<1$ implies that all eigenvalues of $D \tilde{W}^{k}\left(q_{1}^{*}\right)$ have a modulus less than 1 . These eigenvalues are the solutions of the characteristic equation

$$
\operatorname{det}\left(D \tilde{W}^{k}\left(q_{1}^{*}\right)-\lambda I\right)=0
$$

in which $I$ is this time the unit $(T, T)$ matrix. It is known that given the specific form of the Jacobian matrix $D \tilde{W}^{k}\left(q_{1}^{*}\right)$ shown above, this characteristic equation may be written equivalently $\operatorname{det}(B(\lambda))=0$, in which $B(\lambda)$ is the $(k, k)$ matrix that is equal to ${ }^{1}$

$$
B(\lambda)=\lambda^{m} I-\sum_{n=1}^{m} \lambda^{m-n} A_{n}
$$

(here I denotes the $(k, k)$ unit matrix). We claim

(A.10) The characteristic equation of $D \tilde{W}^{k}\left(q_{1}^{*}\right)$ has the form

$$
\lambda^{\top}-\sum_{j=1}^{T} \beta_{j} \lambda^{T-j}=0
$$

in which all $\beta_{j}$ have the same sign and $\sum_{j} \beta_{j}=D \Phi^{k}\left(p_{i}^{*}\right)$.

The claim is in fact immediate when $k=1$. In that case, $m=T$ and since the characteristic equation reads $\operatorname{det}(B(\lambda))=0$, we have $\beta_{j}=W_{1, j+1}=W_{j}^{\prime}\left(q^{*}\right)$, in which $q^{*}$ is the fixed point of $\tilde{W}$ under cons $i-$ deration. Then, in view of $(A .5)$, all $B_{j}$ have the same sign and $\sum_{j} \beta_{j}=\Phi^{\prime}\left(p^{*}\right)$, with $p^{*}$ being the associated fixed point of $\Phi$, i.e. $q^{*}=\left(p^{*}, p^{*}, \ldots\right)$.

I See Wilkinson (1965, pp. 33-34). 
We turn now to the more complex case in which $k \geqq 2$. By employing the expressions of the matrices $A_{n}$ given above, we obtain

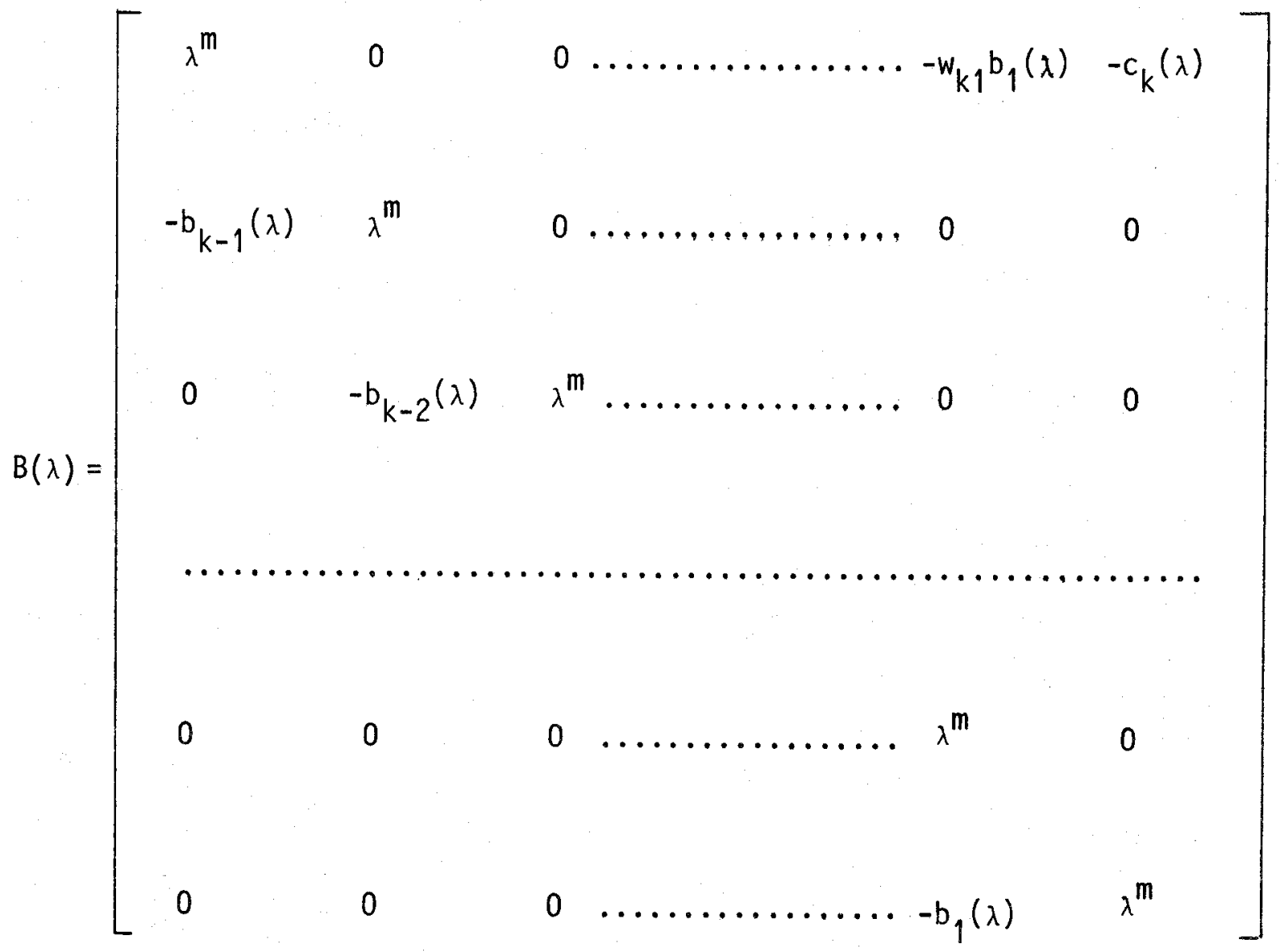

with, for $i=1, \ldots, k$

$$
b_{i}(\lambda)=\sum_{n=1}^{m} w_{i n} \lambda^{m-n}
$$

and

$$
c_{k}(\lambda)=\sum_{n=1}^{m} w_{k, n+1} \lambda^{m-n}
$$

We may develop $\operatorname{det}(B(\lambda))$ with respect to the elements of its first column. We get then 


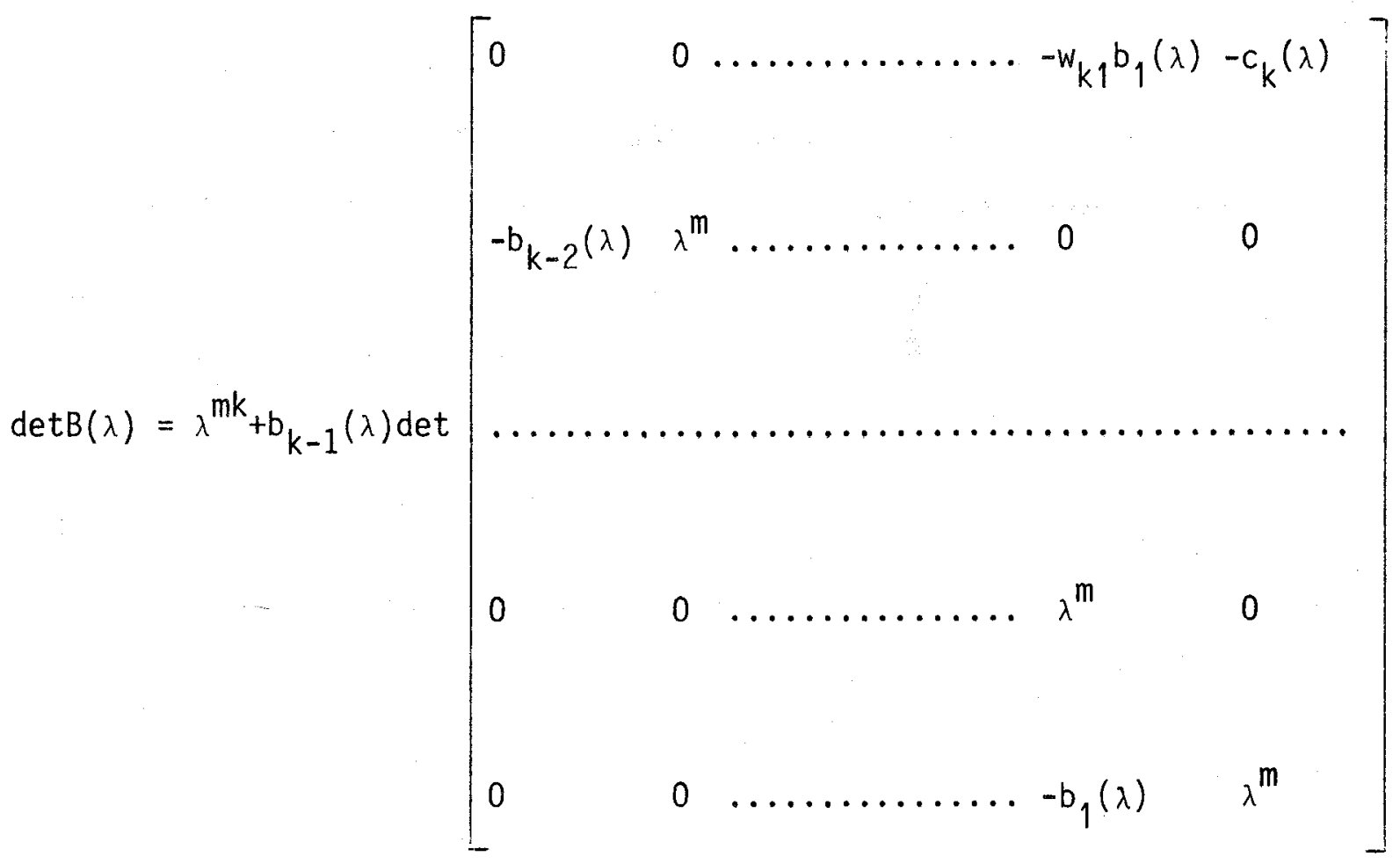

By pursuing along this line, we obtain finally

$$
\operatorname{det}(B(\lambda))=\lambda^{m k}+b_{k-1}(\lambda) \ldots b_{2}(\lambda) \operatorname{det}\left[\begin{array}{cc}
-w_{k 1} b_{1}(\lambda) & -c_{k}(\lambda) \\
-b_{1}(\lambda) & \lambda^{m}
\end{array}\right]
$$

and thus

$$
\operatorname{det}(B(\lambda))=\lambda^{m k}-b_{k-1}(\lambda) \ldots b_{1}(\lambda)\left(w_{k 1} \lambda^{m}+c_{k}(\lambda)\right)
$$

If we recall that $w_{k, m+1}=0$ whenever $k \geqq 2$, we see that

$$
\operatorname{det}(B(\lambda))=\lambda^{m k}-\lambda b_{1}(\lambda) \ldots b_{k}(\lambda)
$$

or equivalently

(A.11) $\quad \operatorname{det}(B(\lambda))=\lambda^{m k}-\lambda\left(\sum_{n=1}^{m} w_{1 n} \lambda^{m-n}\right) \ldots\left(\sum_{n=1}^{m} w_{k n} \lambda^{m-n}\right)$ 
This expression shows clearly that $\lambda b_{1}(\lambda) \ldots b_{k}(\lambda)$ is a polynome in $\lambda$ of degree $(m-1) k+1$, which is less than $T=m k$ when $k \geqq 2$. Thus in order to show (A.10), we must show that the coefficients of the polynome

$$
b_{1}(\lambda) \ldots b_{k}(\lambda)=\left(\sum_{n=1}^{m} w_{1 n} \lambda^{m-n}\right) \ldots\left(\sum_{n=1}^{m} w_{k n} \lambda^{m-n}\right)
$$

have all the same sign, and that their sum is equal to

$$
D \Phi^{k}\left(p_{j}^{*}\right)=\Phi^{\prime}\left(p_{1}^{*}\right) \ldots \Phi^{\prime}\left(p_{k}^{*}\right)
$$

Now the coefficient of $\lambda^{k m-j}$ in $b_{1}(\lambda) \ldots b_{k}(\lambda)$, for $j=k, \ldots, k m$, is the sum of al1 products of the form $w_{1 n_{1}} \ldots w_{k n_{k}}$ such that $n_{1}+\ldots+n_{k}=j$, with $m \geqq n_{i} \geqq 1$ for all $i=1, \ldots, k$. Since $w_{i n}=w_{n k-1}^{\prime}\left(q_{j}^{*}\right)$, in view of $(A .5)$, each such product has the same sign as $D_{\Phi}{ }^{k}\left(p_{j}^{*}\right)$. Thus all coefficients of the polynome $b_{1}(\lambda) \ldots b_{k}(\lambda)$ have the same sign. Next, their sum is equal to

$$
\begin{aligned}
\sum_{n_{1}=1}^{m} \cdots \sum_{n_{k}=1}^{m} w_{1 n_{1}} \cdots w_{k n_{k}} & =\left(\sum_{n=1}^{m} w_{1 n}\right) \ldots\left(\sum_{n=1}^{m} w_{k n}\right) \\
& =\Phi^{\prime}\left(p_{1}^{*}\right) \ldots \Phi^{\prime}\left(p_{k}^{*}\right) \\
& =D^{k}\left(p_{j}^{*}\right)
\end{aligned}
$$

This completes the proof of $(A .10)$. The last step of the proof of Proposition is to show that $\left|D_{\Phi}{ }^{k}\left(p_{j}^{*}\right)\right|<1$ implies that all solutions of the characteristic equation of $D \tilde{W}^{k}\left(q_{1}^{*}\right)$ have a modulus less than 1. By virtue of $(A .10)$, the characteristic equation of $D \tilde{W}^{k}\left(q_{1}^{*}\right)$ reads 


$$
\lambda^{\top}-\sum_{j=1}^{T} \beta_{j} \lambda^{T-j}=0
$$

in which all $\beta_{j}$ have the same sign and $\sum_{j} \beta_{j}=D \Phi^{k}\left(p_{j}^{*}\right)$. Let us assume $\left|D \Phi^{k}\left(p_{j}^{*}\right)\right|<1$. This implies

$$
\left|\sum_{j} \beta_{j}\right|=\sum_{j}\left|\beta_{j}\right|<1
$$

Now, suppose that there exists a particular eigenvalue $\bar{\lambda}$ that has a modulus $|\bar{\lambda}|$ greater than or equal to 1 . Since

$$
|\bar{\lambda}|^{\top}=\left|\sum_{j} \beta_{j} \bar{\lambda}^{T-j}\right| \leqq \sum_{j}\left|\beta_{j}\right||\bar{\lambda}|^{T-j}
$$

one gets by dividing by $|\bar{\lambda}|^{\top}$

$$
1 \leqq \sum_{j}\left|\beta_{j}\right||\bar{\lambda}|^{-j} \leqq \sum_{j}\left|\beta_{j}\right|
$$

which yields a contradiction to the assumption $\left|D \Phi^{k}\left(p_{j}^{*}\right)\right|<1$. The proof of Proposition 3.1 is complete. 
B. EXISTENCE, STABILITY AND BIFURCATION OF PERIODIC EQUILIBRIA.

The aim of this Section is to prove some of the claims made in Section 4, namely Theorems 4.3, 4.5 and 4.7. The arguments will be direct applications of the results reported in Collet and Eckmann (1980), as reviewed in Grandmont (1983b).

Proof of Theorem 4.3. Under the assumptions of this Theorem, if $\alpha_{2}=\operatorname{SupR}_{2}\left(a_{2}\right) \leqq 1$, or if $\alpha_{2}>1$ and $\theta^{*} \geqq 1$, then $\varphi$ has a unique monetary cycle $\theta=1$ by Lemma 4.2. If $\theta^{*}<1$, then $\varphi(\theta) \leqq \varphi\left(\theta^{*}\right)$ for all $\theta \geqq \bar{\theta}$, and we may focus attention without any loss of generality to those $\theta$ which belong to $\left[\bar{\theta}, \varphi\left(\theta^{*}\right)\right]$. But the restriction of $\varphi$ to that interval satisfies the assumptions of Sarkovskii's Theorem (Collet and Eckmann, 1980, Theorem II.3.10, Grandmont, 1983b, Theorem 1).

Proof of Theorem 4.5. Under the assumptions of this Theorem, the restriction of $\varphi$ (or of one of its topological conjugates $\tilde{\varphi}=h \circ \varphi \circ h^{-1}$ ) to the interval $\left[\bar{\theta}, \varphi\left(\theta^{*}\right)\right]$ (or to $\left[h(\bar{\theta}), \tilde{\varphi}\left(h\left(\theta^{*}\right)\right)\right]$ ) is thrice continuously differentiable, has a unique maximum at $\theta^{*}$ with $\bar{\theta}<\theta^{*}<\varphi\left(\theta^{*}\right)$, and $S \varphi(\theta)<0$ for $a 11 \theta$ in $\left[\bar{\theta}, \varphi\left(\theta^{*}\right)\right], \theta \neq \theta^{*}$ (or the corresponding property for $\tilde{\varphi})$. Moreover, $\varphi(\theta)>\theta$ for all $\theta$ in $\left(\bar{\theta}, \theta^{*}\right), \varphi(\bar{\theta})=\bar{\theta}$ and $\varphi^{\prime}(\bar{\theta})>1$. Thus Theorem 2 in Grandmont (1983b) applies to $\varphi$ or $\tilde{\varphi}$. The proof is completed if one remarks that $\varphi(\theta)$ belongs to $\left[\bar{\theta}, \varphi\left(\theta^{*}\right)\right]$ whenever $\theta \geqq \bar{\theta}$. Q.E.D. 
Proof of Theorem 4.7. Here again, we may focus attention on the interval $A_{\lambda}=\left[\bar{\theta}_{\lambda}, \varphi_{\lambda}\left(\theta_{\lambda}^{*}\right)\right]$ for each $\lambda$. The result is then a direct application of Theorems 7 and 9 in Grandmont (1983b). Indeed it is routine to verify that if one looks at the restrictions of $\varphi_{\lambda}$ to $A_{\lambda}$, one gets a full oneparameter family of $C^{1}$-unimodal maps that satisfies $S 1, S 2, S 3, S 4$ " for each $\lambda$, as these conditions are defined in Grandmont (1983b). As for the condition $\varphi_{\lambda}^{\prime \prime}\left(\theta_{\lambda}^{*}\right)<0$ it is not difficult to verify that it is a topological invariant (see the Section on topological conjugacy in Grandmont 1983b), so that it is satisfied if and only if $x_{\lambda}^{\prime \prime}\left(\mu_{\lambda}^{*}\right)<0$. Dropping the index $\lambda$ for simplicity, we get by differentiating twice $v_{1}(x(\mu))=v_{2}(\mu)$ and by taking into account $x^{\prime}\left(\mu^{*}\right)=0$,

$$
x^{\prime \prime}\left(\mu^{*}\right)=v_{2}^{\prime \prime}\left(\mu^{*}\right) / v_{1}^{\prime}\left(x\left(\mu^{*}\right)\right)
$$

Since $v_{1}^{\prime}(\mu)>0$ for all $\mu, \chi^{\prime \prime}\left(\mu^{*}\right)<0$ is equivalent to $v_{2}^{\prime \prime}\left(\mu^{*}\right)<0$. Now by differentiation of $v_{2}(\mu)=\mu V_{2}^{\prime}\left(e_{2}^{*}+\mu\right)$, we obtain

$$
v_{2}^{\prime}(\mu)=V_{2}^{\prime}\left(e_{2}^{\star}+\mu\right)_{\rho}(\mu)
$$

where

$$
\rho(\mu)=1-\frac{\mu}{l_{2}^{*}+\mu} R_{2}\left(l_{2}^{*}+\mu\right)
$$

Differentiating again,

$$
v_{2}^{\prime \prime}\left(\mu^{*}\right)=V_{2}^{\prime}\left(e_{2}^{*}+\mu^{*}\right) \rho^{\prime}\left(\mu^{*}\right)<0
$$

since $\rho\left(\mu^{*}\right)=0$ and $\rho^{\prime}(\mu)<0$ under assumption $(1 . e)$. 